\title{
Electrospun PEDOT:PSS Electrolytic Capacitor Electrodes for Microelectronic Energy Storage
}

by

\author{
Colin Fernandes, B.Eng
}

A thesis submitted to the Faculty of Graduate and Postdoctoral Affairs in partial fulfillment of the requirements for the degree of

Master of Applied Science

in

Electrical and Computer Engineering

Ottawa-Carleton Institute for Electrical and Computer Engineering

Department of Electronics

Carleton University

Ottawa, Ontario, Canada

(c) 2018, Colin Fernandes 


\begin{abstract}
This thesis investigates the use of electrospinning process as an additive fabrication method to deposit porous ultrahigh surface area poly(3,4-ethylenedioxythiophene):polystyrene sulfonate (PEDOT:PSS) as electrodes for use in electrolytic capacitors for powering microelectronic circuits. An electrospinning process for depositing high PEDOT content fibres is established using Baytron P/Poly(ethlyene Oxide)/Dimethyl Sulfoxide. The electrospun fibre mats are utilized as both the anode and cathode with Lithium Perchlorate ( $\mathrm{LiClO} 4) /$ Propylene Carbonate (PC) as the electrolyte. This work investigates the electrochemical operation, charge storage metrics, and lifecycle through cyclic voltammetry, DC charging, and electrochemical impedance spectroscopy. Results demonstrate non-faradaic charge storage dominates up to $1.1 \mathrm{~V}$ with a maximum achieved areal capacitance and energy storage of $5.25 \mathrm{mF} \mathrm{cm}^{-2}$ and $3.18 \mathrm{~mJ} \mathrm{~cm}^{-2}$ respectively. The lifecycle investigation finds a $4 \%$ capacitance increase after $20,0001.1 \mathrm{~V}$ cycles at $0.5 \mathrm{~Hz}$.
\end{abstract}




\section{Acknowledgements}

First, I would like to gratefully acknowledge my supervisors Dr. Leonard MacEachern and Dr. Steven P. McGarry. I am extremely thankful for the support and tutelage generously given to me during the course of my studies. I would especially like to express my sincerest gratitude for the patience, technical insight, and overall mentorship I received. I would like to additionally thank Dr. Tom Smy for being a significant source of academic guidance throughout my studies.

I would also like to thank Blazenka Power, Rob Vandusen, Nagui Mikhail, and all the wonderful members of the DOE that provided me with resource, tools and guided me through my time at Carleton University.

I would like to thank my family for their endless support, specifically my parents for always checking in and being available when I needed them and the support to help alleviate the health challenges faced throughout my degree. Most importantly the memory of Grant Bell, I would not be here today conducting work I love without your inspiration and life long influence.

I am extremely thankful to my friends for being the inertia needed to push through the tough times. Specifically David Berton and Aaron English I will always value the numerous times your refreshing perspective, and approach to your respective work provided inspiration and motivation for mine. Finally, I am grateful for the teammates, and coaches who enhanced my personal growth and experience through displays of expert leadership and guidance during my tenure playing for Carleton. 


\section{Symbols}

\begin{tabular}{|c|c|}
\hline$M_{w}$ & Molecular Weight \\
\hline$\nu$ & Flory's Exponent \\
\hline$N_{A}$ & Avogadro's Number \\
\hline $\mathrm{V}$ & Electric Potential [V] \\
\hline$\omega$ & Angular Frequency $[\mathrm{rad} / \mathrm{s}]$ \\
\hline$\Omega$ & Ohms \\
\hline$\alpha$ & Constant Phase Element Exponent [unitless] \\
\hline$Q_{i}$ & Constant Phase Element Coefficient $\left[S s^{\alpha}\right]$ \\
\hline $\mathrm{C}$ & Capacitance $[\mathrm{F}]$ \\
\hline $\mathrm{R}$ & Resistance $[\Omega]$ \\
\hline $\mathrm{Z}_{i}$ & Complex Impedance $[\Omega+\mathrm{i} \Omega]$ \\
\hline $\mathrm{Q}_{W}$ & Warburg Coefficient $\left[S s^{\frac{1}{2}}\right]$ \\
\hline $\mathrm{D}$ & Diffusion Coefficient $[2 / \mathrm{s}]$ \\
\hline$\delta$ & Nernst Diffusion Layer Thickness [m] \\
\hline $\mathrm{RH}$ & Relative Humidity \\
\hline RT & Room Temperature \\
\hline$\lambda_{D E B Y E}$ & Debye Length [m] \\
\hline $\mathrm{M}$ & Molarity \\
\hline
\end{tabular}




\section{Nomenclature}

\begin{tabular}{|c|c|}
\hline PEDOT:PSS & Poly(3,4-ethylenedioxythiophene):Poly(4-styrenesulfonate) \\
\hline EDOT & Ethylenedioxythiophene monomer \\
\hline PSS & Poly(4-styrenesulfonate) \\
\hline PVA & Poly(vinyl alcohol) \\
\hline $\mathrm{PEO}$ & Poly(ethylene oxide) \\
\hline $\mathrm{CP}$ & Conducting Polymer \\
\hline $\mathrm{EC}$ & Electrochemical Capacitor \\
\hline $\mathrm{PsC}$ & Pseudocapacitance \\
\hline EDLC & Electrolytic Double Layer Capacitor \\
\hline $\mathrm{W}$ & Warburg Element \\
\hline EDL & Electrolytic Double Layer \\
\hline IHP & Inner Helmholtz Plane \\
\hline $\mathrm{OHP}$ & Outer Helmholtz Plane \\
\hline $\mathrm{CPE}$ & Constant Phase Element \\
\hline ESR & Equivalent Series Resistance \\
\hline CEA & Conductivity Enhancement Agent \\
\hline $\mathrm{Li}^{+}$ & Lithium \\
\hline $\mathrm{ClO}_{4}^{-}$ & Perchlorate \\
\hline $\mathrm{LiClO}_{4}$ & Lithium Perchlorate \\
\hline $\mathrm{PC}$ & Propylene Carbonate \\
\hline $\mathrm{EtOH}$ & Ethanol \\
\hline EG & Ethlyene Glycol \\
\hline DMSO & Dimethyl Sulfoxide \\
\hline
\end{tabular}


ES Electrospun

CD Charge Discharge

CV Cyclic Voltammetry

IS Impedance Spectroscopy

EIS Electrochemical Impedance Spectroscopy

SEM Scanning Electron Microscopy

DUT Device Under-Test

LMA Levenberg-Marquardt algorithm

GA Genetic Algorithm 


\section{Contents}

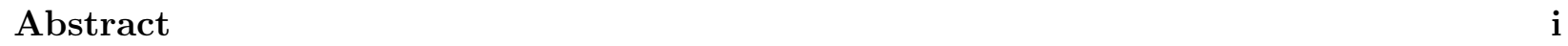

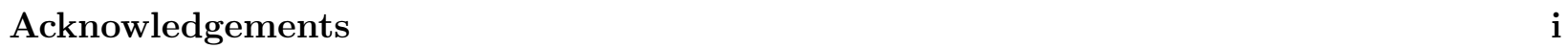

Symbols

Nomenclature

1 Motivation and Background 1

1.1 Thesis Objectives $\ldots \ldots \ldots \ldots \ldots \ldots \ldots \ldots$

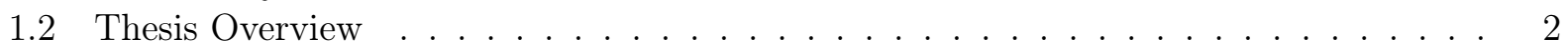

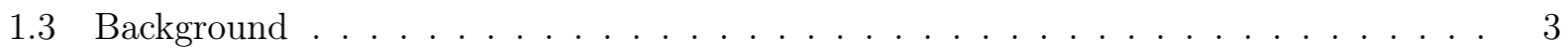

1.3 .1 Microscale Energy Storage . . . . . . . . . . . . . . . . . . . . . . 3

1.3.2 $\quad$ EDLCs: Challenges and the State of the Field . . . . . . . . . . . . . . . . 6

1.3 .3 Electrospun Electrodes . . . . . . . . . . . . . . . . . . 7

1.4 Thesis Contributions $\ldots \ldots \ldots \ldots \ldots \ldots$

$\begin{array}{lll}2 & \text { Background } & 10\end{array}$

2.1 Principles of Polymers . . . . . . . . . . . . . . . . . . . . . . . 10

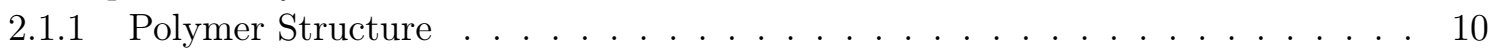

2.2 PEDOT:PSS : A Conducting Polymer . . . . . . . . . . . . . . . . . . . . 13

$2.2 .1 \quad$ Introduction $\ldots \ldots \ldots \ldots \ldots \ldots \ldots \ldots$

$2.2 .2 \quad$ Structure of PEDOT:PSS $\ldots \ldots \ldots \ldots \ldots \ldots \ldots \ldots$

$2.2 .3 \quad$ Solvent Conductivity Effects . . . . . . . . . . . . . . . . . . . . 15

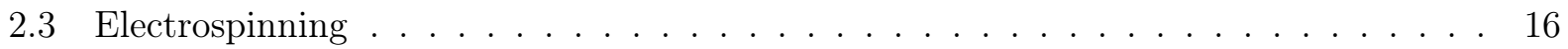

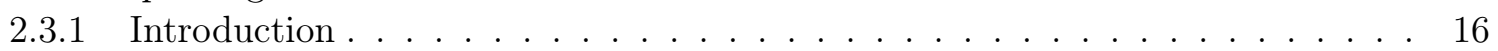

2.3 .2 The Principles of Electrospinning . . . . . . . . . . . . . . . . . . . . . 16

2.3.3 Electrospinning Parameters . . . . . . . . . . . . . . . . . . . . . . . . 18

2.4 Electrolytic Capacitors . . . . . . . . . . . . . . . . . . . . . . . . . . . . 19

$2.4 .1 \quad$ Introduction $\ldots \ldots \ldots \ldots \ldots \ldots$

$2.4 .2 \quad$ Electrode-Electrolyte Processes . . . . . . . . . . . . . . . . . . . . 20

$2.4 .3 \quad$ Electrolyte Processes $\ldots \ldots \ldots \ldots \ldots \ldots$. . . . . . . . . . . . . . . . . 22

2.4 .4 Summary . . . . . . . . . . . . . . . . . . . . . 24

2.5 Electrochemical Capacitor Investigation Techniques . . . . . . . . . . . . . . . . 25

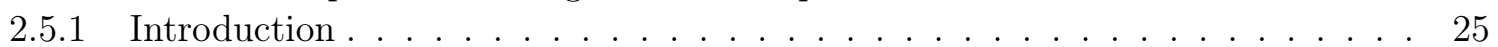

$2.5 .2 \quad$ DC Charging . . . . . . . . . . . . . . . . . . . . . . . 26

$2.5 .3 \quad$ Cyclic Voltammetry $\ldots \ldots \ldots \ldots \ldots \ldots$ 
$2.6 \quad$ Electrochemical Impedance Spectroscopy $\ldots \ldots \ldots$. . . . . . . . . . . . . . . . . . . 28

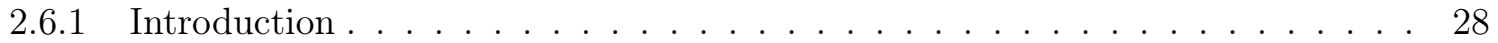

$2.6 .2 \quad$ EIS Data Representation $\ldots \ldots \ldots \ldots$. . . . . . . . . . . . . 29

$2.6 .3 \quad$ Equivalent Circuit Modeling of Electrochemical Systems . . . . . . . . . . . . 30

2.6 .4 ECM Models . . . . . . . . . . . . . . . . . . . . . . . . . . . . . . 31

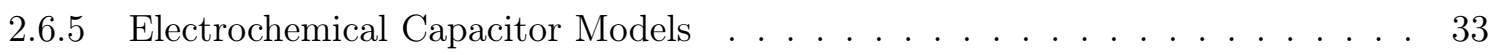

2.6 .6 EIS data fitting . . . . . . . . . . . . . . . . . . . . . . . . . . . 34

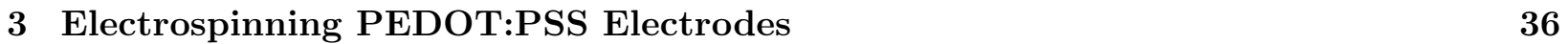

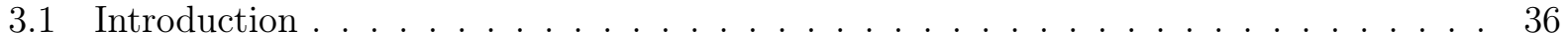

3.2 Experimental Setup and Method . . . . . . . . . . . . . . . . . . 37

$3.2 .1 \quad$ Electrospinning Apparatus . . . . . . . . . . . . . . . . . . . . . 37

3.2 .2 Electrospinning Method . . . . . . . . . . . . . . . . . . . . . . . 38

3.3 Initial Process Tuning $\ldots \ldots \ldots \ldots$. . . . . . . . . . . . . . . . . . . . . 40

3.4 PEDOT:PSS Conductive Nanofibres $\ldots \ldots \ldots \ldots \ldots$. . . . . . . . . . . . . 41

$3.4 .1 \quad$ Solution Tuning . . . . . . . . . . . . . . . . . . . . . . . . . . . 41

3.4 .2 Post-Processing . . . . . . . . . . . . . . . . . . . . . . . . 43

3.5 Optimization: PEDOT:PSS $/ \mathrm{PEO} \ldots \ldots \ldots \ldots \ldots \ldots \ldots$

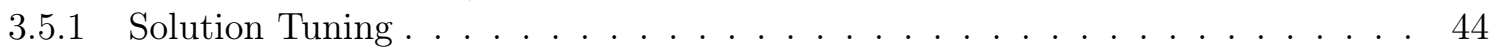

3.6 Summary of Process $\ldots \ldots \ldots \ldots \ldots \ldots \ldots$

\begin{tabular}{|lll}
4 & EDLC Test Structure & 49
\end{tabular}

4.1 Components . . . . . . . . . . . . . . . . . . . . . . . . . . . . 49

4.2 Test Structure Geometry and Preparation . . . . . . . . . . . . . . . . . . . . 51

5 Electrospun PEDOT:PSS/PVA/PEO EC Performance Analysis $\quad 54$

5.1 Introduction $\ldots \ldots \ldots \ldots \ldots \ldots \ldots$

5.2 Cyclic Voltammetry: Method and Result . . . . . . . . . . . . . . . . . 55

$5.2 .1 \quad$ Experimental Setup $\ldots \ldots \ldots \ldots \ldots \ldots$

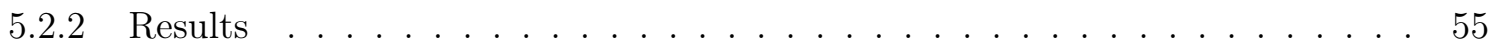

5.3 DC Charging: Experimental Setup and Results . . . . . . . . . . . . . . . . . 58

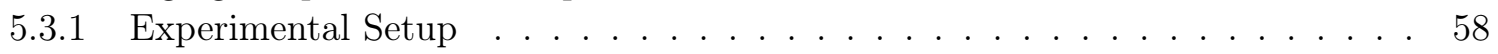

$5.4 \quad$ Electrochemical Impedance Spectroscopy of PEDOT:PSS/PVA EC . . . . . . . . . . 62

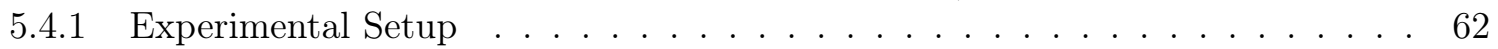

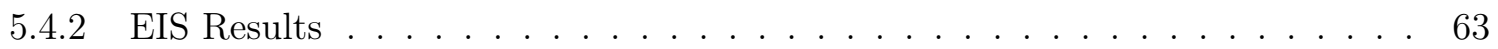

$5.4 .3 \quad$ Equivalent Circuit Modelling . . . . . . . . . . . . . . . . . . . . 64

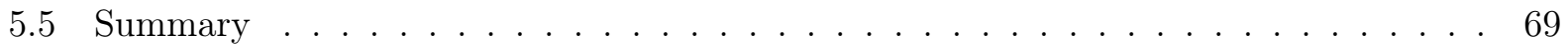

6 Electrospun PEDOT:PSS/PEO EC

$\begin{array}{ll}\text { Performance Analysis } & \mathbf{7 1}\end{array}$

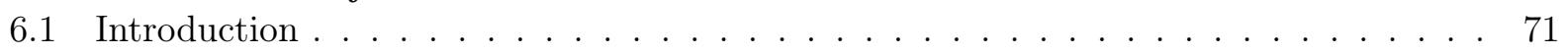

6.2 Cyclic Voltammetry: PEDOT:PSS/PEO . . . . . . . . . . . . . . . . 72

6.3 DC Charging: PEDOT:PSS $/ \mathrm{PEO} \ldots \ldots \ldots \ldots \ldots$ 
6.4 Electrochemical Impedance Spectroscopy of PEDOT:PSS/PEO EC . . . . . . . . . . 75

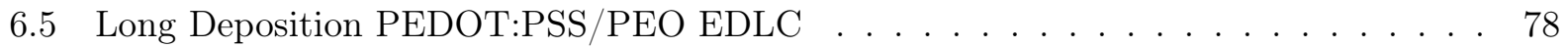

6.5 .1 Introduction . . . . . . . . . . . . . . . . . . 78

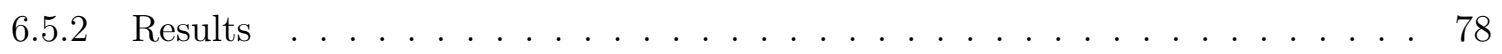

6.6 Conclusion . . . . . . . . . . . . . . . . . . . . . . . 82

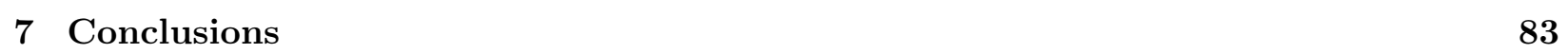

7.1 Summary of Results . . . . . . . . . . . . . . . . . . . . . . . 83

7.1 .1 Comparison to State-of-the-art Literature . . . . . . . . . . . . . . . . . 84

7.2 Contributions to the Field . . . . . . . . . . . . . . . . . . . . . . 86

7.3 Future Work and Improvements $\ldots \ldots \ldots \ldots$

\begin{tabular}{lr}
\hline References & 89
\end{tabular} 


\section{List of Tables}

1.1 Summary of some state-of-the-art materials and their metrics. . . . . . . . . . . . . 7

2.1 Equivalent circuit model components . . . . . . . . . . . . . . . . . . 31

3.1 Summary of PEO and PVA ES solution tuning . . . . . . . . . . . . . . . . 40

3.2 Proporational Entanglement concentrations of PEO and PVA . . . . . . . . . . . . 41

3.3 Viable ES PEO and PVE concentrations . . . . . . . . . . . . . . . . . . . . 41

3.4 Electrospinning apparatus parameters for PEDOT:PSS/PVA/PEO solution for con-

ductive nanofibres. . . . . . . . . . . . . . . . . . . . . . . . 42

3.5 ES PEDOT:PSS/PEO/DMSO solution tuning results $\ldots \ldots \ldots$. . . . . . . . . . 45

3.6 ES fibre diameter and distribution results $\ldots \ldots \ldots \ldots$. . . . . . . . . . . . . 47

3.7 Summary of final ES apparatus parameters . . . . . . . . . . . . . . . . . . . . 47

3.8 Post-processing steps $\ldots \ldots \ldots \ldots \ldots \ldots \ldots$

$5.1 \quad$ PEDOT:PSS/PVA/PEO CV results $\ldots \ldots \ldots \ldots \ldots \ldots$

5.2 PEDOT:PSS/PVA/PEO EDLC performance metrics . . . . . . . . . . . . . . . 60

5.3 ECM parameter overview $\ldots \ldots \ldots \ldots \ldots$

$5.4 \quad$ ECM fit parameters for control and PEDOT:PSS/PVA/PEO EC $\ldots \ldots \ldots$. . . . . 69

$6.1 \quad$ DC charging results for PEDOT:PSS/PEO $1.1 \mathrm{~V} \ldots \ldots \ldots \ldots$

$6.2 \quad$ ECM fit parameters for ES PEDOT:PSS/PEO $\ldots \ldots \ldots \ldots$. . . . . . . . . . . . 77

$6.3 \quad$ Thick electrode PEDOT:PSS/PVA DC charging results at $V_{S}=1.1 \mathrm{~V} \ldots \ldots$

$6.4 \quad$ ECM Fit parameters for the thick deposition of PEDOT:PSS/PEO fibres . . . . . 81

7.1 Summary of final ES apparatus parameters $\ldots \ldots \ldots$. . . . . . . . . . . . 83

7.2 Summary of EC performance for all stages $\ldots \ldots \ldots \ldots$. . . . . . . . . . 84

7.3 Comparison to state-of-the-art literature $\ldots \ldots \ldots \ldots$. . . . . . . . . . . 85 


\section{List of Figures}

1.1 ELDC test structure illustration $\ldots \ldots \ldots \ldots \ldots \ldots \ldots \ldots$

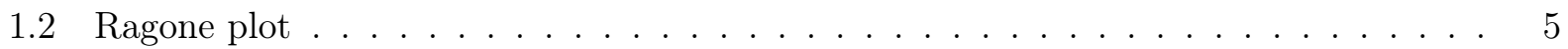

1.3 Families of electrolytic capacitors $\ldots \ldots \ldots \ldots \ldots$

2.1 PEO and PVA monomers . . . . . . . . . . . . . . . . . . . . . 11

2.2 Illustration of the PEDOT:PSS copolymer. . . . . . . . . . . . . . . . . 14

2.3 PEDOT:PSS Conformation Diagram . . . . . . . . . . . . . . . . . . . 15

2.4 Electrospinning apparatus diagram $\ldots \ldots \ldots \ldots \ldots$. . . . . . . . . . . . . 17

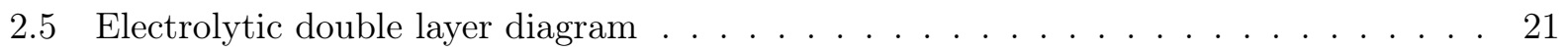

2.6 Basic equivalent Circuit model of EDLC $\ldots \ldots \ldots \ldots \ldots$

$2.7 \quad$ EIS data representation examples . . . . . . . . . . . . . . . . . . . . . . . . . . 30

2.8 Randles Circuit . . . . . . . . . . . . . . . . . . . . . . . . . . . . . . . . . . . . . 31

2.9 EDL diagram with equivalent circuit model $\ldots \ldots \ldots$. . . . . . . . . . . . 32

2.10 Ideal electrolytic capacitor ECM model with adsorption and interface resistance. . . 34

2.11 State-of-the-art model presented for highly porous EDLC electrodes shown first by

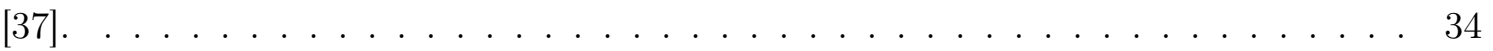

3.1 Photo of ES apparatus $\ldots \ldots \ldots \ldots \ldots \ldots$

$3.2 \quad$ Electrospinning method flow chart $\ldots \ldots \ldots \ldots$

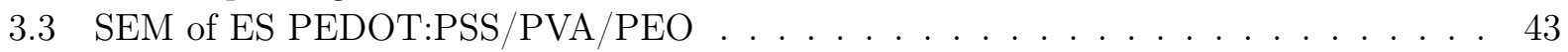

$3.4 \quad$ SEM image of DMSO treated PEDOT:PSS/PVA/PEO fibre . . . . . . . . . . . . . 43

3.5 ES PEDOT:PSS/PEO SEM Results $\ldots \ldots \ldots$. . . . . . . . . . . . . . 46

$4.1 \quad$ ELDC test structure illustration $\ldots \ldots \ldots \ldots \ldots \ldots$

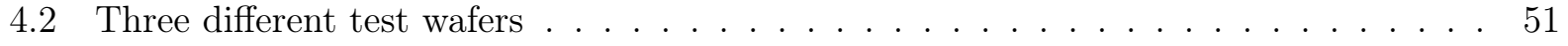

4.3 Photos of the wafers before and after ES collection. . . . . . . . . . . . . . . 51

4.4 Close up photo of a test structure after ES fibre collection . . . . . . . . . . . . . . . 52

$4.5 \quad$ Final processing steps. . . . . . . . . . . . . . . . . . . . 53

$5.1 \quad$ CV control results $\ldots \ldots \ldots \ldots \ldots \ldots \ldots$

5.2 CV of ES PEDOT:PSS/PVA/PEO and control . . . . . . . . . . . . . . . . 56

$5.3 \quad$ CV voltage window investigation $\ldots \ldots \ldots \ldots \ldots \ldots$

5.4 Charging period vs capacitance of PEDOT:PSS/PVA/PEO EDLC $\ldots \ldots \ldots . . .58$

$5.5 \quad 1.1 \mathrm{~V} \mathrm{DC}$ charging PEDOT:PSS/PVA $/ \mathrm{PEO} \ldots \ldots \ldots \ldots \ldots$

5.6 Galvanostatic cycling of PEDOT:PSS/PVA $/ \mathrm{PEO} \ldots \ldots \ldots \ldots$

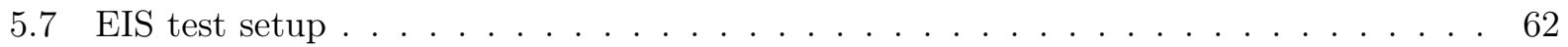

5.8 Nyquist plots for PEDOT:PSS/PVA/PEO and control EIS . . . . . . . . . . . . . 63

5.9 Visual representation of the spread of solutions for the control fit for 200 different simulations through GA and LMA all which met the residual criterion of the result.

The $\mathrm{x}$ axis is the value/mean to show the \% deviation of the data fitting result. . . . 67 5.10 EIS of control, left, and PEDOT:PSS/PVA/PEO, right, with ECM data fitting results. 68 
6.1 Voltage window and sweep rate CV of PEDOT:PSS/PEO . . . . . . . . . . . 72

6.2 Sweep rate vs areal capacitance of three different sizes of PEDOT:PSS/PEO ECs . . 73

$6.3 \quad 1.1 \mathrm{~V}$ DC charging of PEDOT:PSS/PEO EDLC $\ldots \ldots \ldots \ldots . \ldots 74$

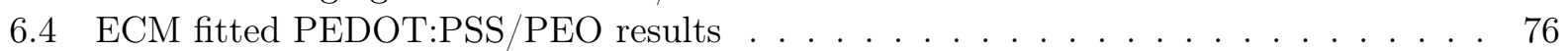

6.5 Thick ES deposition on wafer $\ldots \ldots \ldots \ldots$. . . . . . . . . . . . . . . . . 78

6.6 Summary of final PEDOT:PSS $/$ PEO device results. . . . . . . . . . . . . . . . . . 80 


\section{Chapter 1}

\section{Motivation and Background}

\subsection{Thesis Objectives}

The purpose of this thesis is to investigate electrospun (ES) poly(3,4-ethylenedioxythiophene) :poly(styrenesulfonate) (PEDOT:PSS) as electrodes in electrolytic double layer capacitor (EDLCs) structures as microscale energy storage. The proposed electrodes aim toward autonomous sensor applications and is compatible with conventional silicon integrated chip (IC) processing.

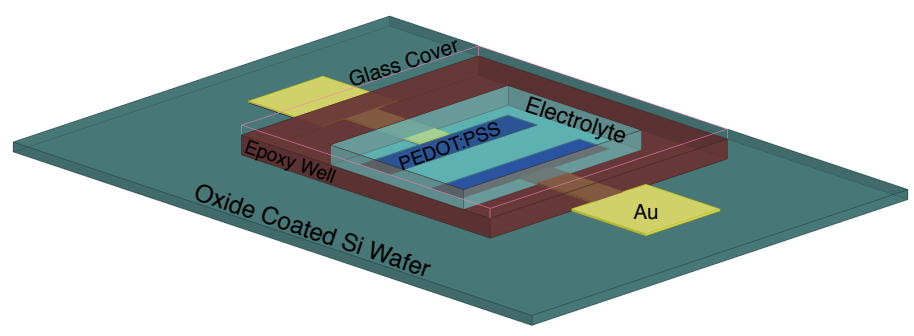

Figure 1.1: Illustration of the EDLC test structure. The structure consists of patterned gold contacts with ES PEDOT:PSS electrodes sealed in a well of electrolyte.

The objectives of the work was achieved through 4 stages: first an electrospinning process to deposit ES PEDOT:PSS fibre electrodes compatible with both silicon and flexible substrates is developed; second the EDLC structures are studied using conventional electrochemical techniques; third complex non-linear least squares (CNLS) fitting of experimental data to an equivalent circuit model of the porous electrodes is used to provide quantitative insight of the performance; and finally the incorporation of dimethyl sulfoxide (DMSO), a standard PEDOT:PSS post-processing solvent, into the process is explored and the energy storage performance metrics are compared against state-ofthe-art supercapacitors. 


\subsection{Thesis Overview}

A brief background to the requirements for microelectronic energy storage devices for autonomous sensors, the design considerations of EDLC devices, and the electrospinning of electrodes is presented in Chapter 1.4. Here the challenges facing thickness scalability and processing restrictions are emphasized while presenting leading microscale supercapacitor technologies in Table 1.1. The chapter concludes with an outline of the motivation and contributions of the work.

Chapter 2 provides an overview of the required theory surrounding experiments conducted within this thesis. The basic structure and properties of polymers and specifics of the conducting polymer PEDOT:PSS are presented. The electrospinning process is then introduced, presenting the control parameters and process requirements of the apparatus and polymer solutions in order to achieve a stable deposition. This is followed by an outline of the function and basic theory about electrolytic double layer capacitors. The motivation and method of equivalent circuit modeling (ECM) of electrochemical systems is described followed by a presentation of EDLC models. With the function and primary mechanisms outlined, the theory and experimental methods surrounding cyclic voltammetery (CV), electrochemical impedance spectroscopy (EIS), and charge-discharge (CDC) analysis of the EDLCs are derived. Finally, a review of the important metrics used within the electrochemical energy storage field are shown.

The development of the conductive ES fibres and electrospinning solution is presented chronologically in Chapter 3. As a starting point; aqueous test solutions containing poly(ethlyene oxide) (PEO) and poly(vinyl alcohol) (PVA) were used to find the operating parameters during construction of the electrospinning apparatus. PEDOT:PSS was then incorporated and the composition ratio was tested until a stable electrospinning was observed, this process was iterated until a conductive PEDOT:PSS ES fibre was obtained. The fabrication methods are followed by an investigation through scanning electron microscope (SEM) and optical microscopy of the resultant ES fibre electrodes.

The remainder of this thesis covers the experimental apparati, methods, analysis and conclusions 
of the different analysis techniques and the extracted metrics and model parameters. Chapter 4 introduces the structures of the test device, the collection substrate, then the electrolyte and construction process of a device. Chapter 5 covers the method, results, data and extracted parameters from CV, EIS, and CDC experiments of ES PEDOT:PSS/PVA/PEO electrodes. In addition, the EIS results are fitted to a ECM for porous electrodes through a genetic algorithm (GA) and complex non-linear least squares (CNLS) data fitting, providing insight to the behavior of the electrode. The results are then used to improve the electrode and in Chapter 6 ES PEDOT:PSS/PEO electrodes are studied as before with the addition of life cycle testing. Finally, Chapter 7 concludes the thesis by summarizing the results, comparing to state-of-the-art EC electrodes, and suggesting future work and improvements.

\subsection{Background}

The three following subsections will provide a brief overview of the research surroundings. First, the requirements of microscale energy storage for autonomous sensors; followed by the design requirements of EDLC electrodes, the materials, and fabrication; and finally, the introduction of electrospinning, the electrode fabrication method used within this thesis, and current research trends regarding ES high surface area electrodes for supercapacitor applications (SC).

\subsubsection{Microscale Energy Storage}

In a data driven economy, the demand for small, independent, data acquisition has led to the reduction in size, power consumption, and cost of sensor focused microelectronics. The search for autonomous energy for these circuits is growing and diversifying. An autonomous energy solution is two-fold: energy harvesting and energy storage. Microelectronic transducers can harvest energy from vibrational, solar, thermal gradient, evaporation, and electromagnetic energy. Unfortunately,

in practice the energy harvesting sources are infrequent and sporadic, requiring a complimentary energy storage system to mediate and supply consistent power. To contextualize energy systems for autonomous sensors, a set of important general metrics are used to describe an ideal autonomous energy storage system [1], [2]: 
- High Cycle-ability: Avoiding high cost or impractical replacement of the energy storage component or the entire autonomous sensors.

- Moderate Energy Density: Infrequent energy availability requires sufficient energy stored to provide operation through intermittent charging.

- High Power Density: The high power bursts from energy harvesting technology would be optimally paired with an energy storage system capable to fast-charge off infrequent bursts.

- Environmental Compatibility: Lightweight, durable to physical fatigue, varying thermal and biocompatibility requirements based on specific application.

- Integrated Chip (IC) Fabrication compatible: All materials, components and additional system components must use existing IC processing techniques or post-process compatible.

Conventionally, localized electrical energy storage has been dominated by modern chemical battery technologies for devices such as cell phones and wearable medical devices. A battery's high energy density and simplicity has led to its commercial success. However, batteries are not the only electrical energy storage system investigated for microelectronics as fuel cell and capacitors are additional families of well-studied energy storage devices [1], [3], [4]. The Ragone plot seen in Figure 1.2 highlights the relationship of these different energy storage systems [5]. The ideal energy storage technology would exist in the top right corner, having no compromise between the amount of energy stored and the rate it can be delivered. 


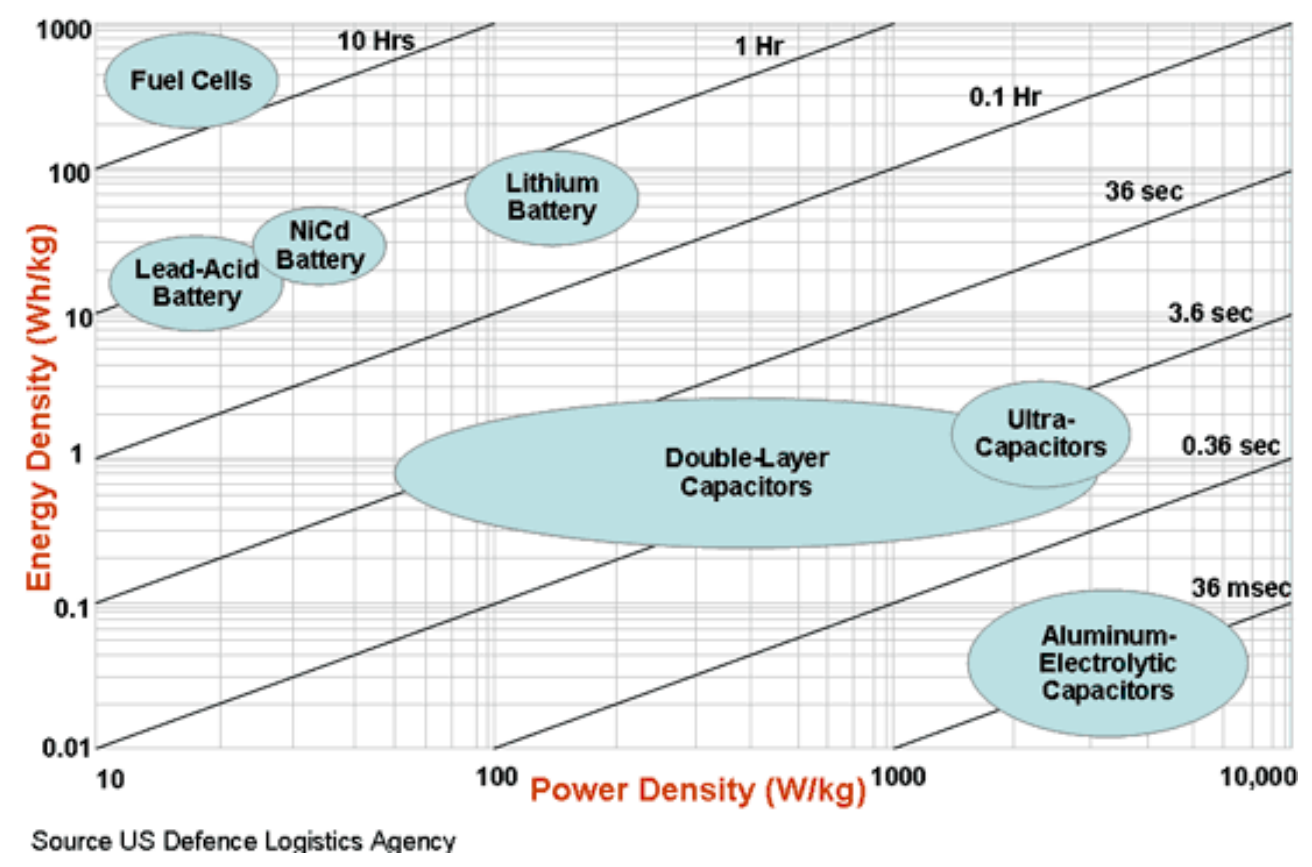

Figure 1.2: Ragone plot showing common commercialized energy storage technologies 5 .

Fuel cells and batteries possess the highest achievable energy densities $\left(\sim 10^{1}-10^{3} \mathrm{Wh} / \mathrm{kg}\right)$. However, surface redox reactions governed by diffusive and faradaic charge-transfer processes constrain charge/discharge capabilities $\left(\sim 10^{1}-10^{2} \mathrm{~W} / \mathrm{kg}\right)$, and limit cycle-ability $\left(\sim 10^{3}\right.$ cycles $)[5],[6]$. Conventional parallel-plate capacitors are the complimentary extreme: high cycle-ability $\left(>>10^{9}\right.$ cycles), high power densities $\left(\sim 10^{4}-10^{6} \mathrm{~W} / \mathrm{kg}\right)$ due to non-faradaic electrostatic storage with low energy density $\left(\sim 10^{-2}-10^{-1} \mathrm{Wh} / \mathrm{kg}\right)$ due to surface area, and dielectric breakdown restrictions. Bridging these two extremes are electrolytic capacitors. Illustrated in Figure 1.3 , electrolytic capacitors exist as a spectrum of devices, storing energy primarily through energy dense, low power faradaic redox reactions known as pseudocapacitance, termed pseudocapacitors, or a high power, lower energy non-faradaic electrolytic double layer (EDL), termed electrolytic double layer capacitors (EDLCs). These devices are generally termed supercapacitors (SCs) 11 (EDLC and SC will be used interchangably throughout this body of work). Practically, all SCs exhibit both faradaic and non-faraaic phenomenon but are classified by their dominate energy storage mechanism. A diverse selection of electrode material and electrolyte has led to wide ranges of achievable energy $\left(10^{0}-10^{1} \mathrm{Wh} / \mathrm{kg}\right)$ and power densities $\left(\sim 10^{1}-10^{3} \mathrm{~W} / \mathrm{kg}\right)$ while maintaining practical lifetimes

\footnotetext{
${ }^{1}$ These structures are referred by many names relatively indiscriminately: ultracapacitors, electrolytic capacitors, electrochemical capacitors (EC), double layer capacitors and EDLC. Throughout this work EDLC and EC will be used for the devices presented in this work and SC as the whole classification of high energy density capacitors.
} 
$>10^{4}$ cycles [5], [7]. The wide range of metrics accessible for EDLCs with non-toxic, biocompatible, and impact resistant materials that are not available to batteries allow EDLCs to be candidates for an autonomous sensor energy storage system.

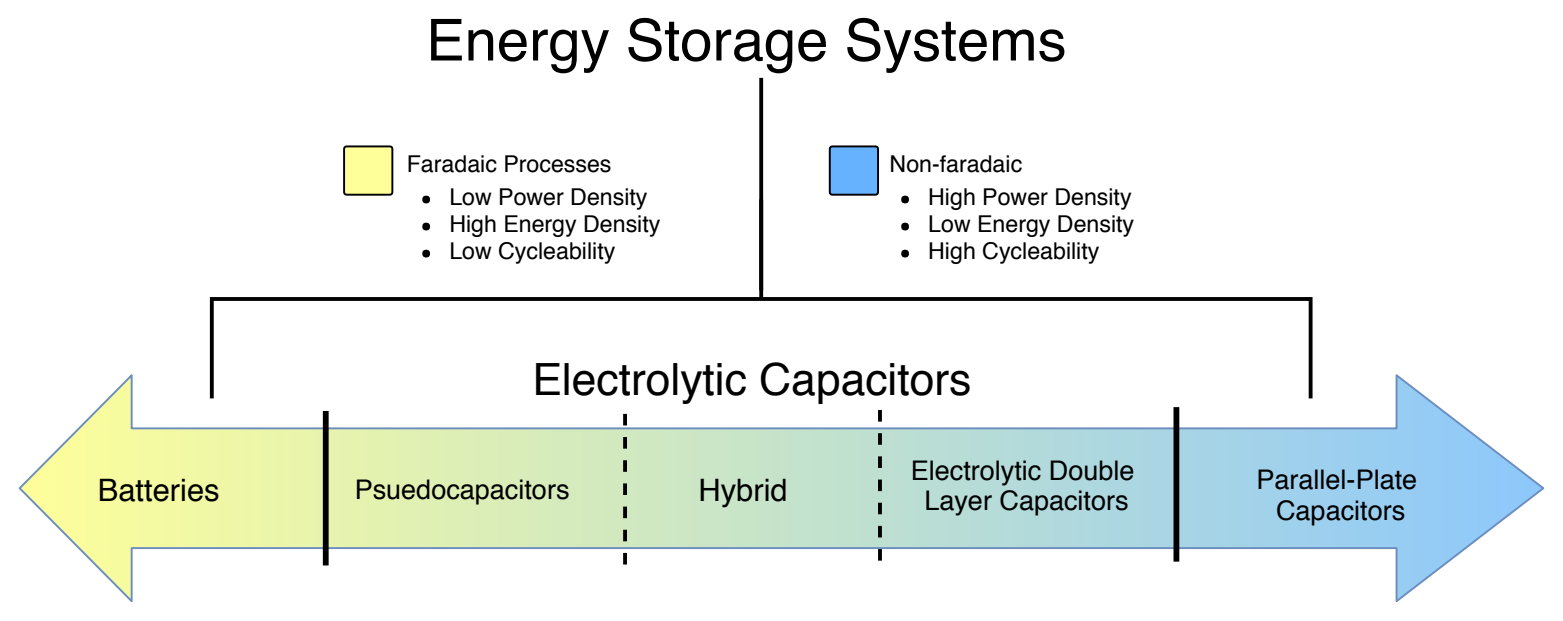

Figure 1.3: Common classifications of electrolytic capacitors and their dominate charge storage mechanism.

\subsubsection{EDLCs: Challenges and the State of the Field}

In order for EDLCs to become an integral component of an autonomous sensor, researchers must overcome a set of challenges: restricting area $2^{2}$ energy density, scalability and cost of electrode fabrication processes, and high internal resistances [1], [7]. An important step in increasing efficiency in EDLC design is to widen the scope and understanding of electrode materials and structures. Various electrode structures using different materials have been explored, as summarized in Table 1.1 . Additionally, on the leading edge the use of complex processing, exotic materials and relying heavily on pseudocapacitance, extremely high energy dense electrodes do exist. Such as a $\mathrm{RuO}_{2} /$ porous $\mathrm{Au}$ at $80 \mu \mathrm{m}$ thick presents an exploratory areal capacitance orders of magnitude higher than other leading materials $\left(\approx 3500 \mathrm{mF} \mathrm{cm}^{-2}\right)$ but the material selection and processing techniques present large potential limitations [1], [8. Improving energy storage through the electrode requires development of ultra high surface area electrodes that are IC process compatible, highly conductive, do not restrict ion flow, and allow for electrolyte wetting of the electrode surface [1], [7], [9]. The relationship between the materials processability and EDLC electrode requirements is extremely limiting for autonomous sensor energy storage systems, as promising electrodes like vacuum filtra-

\footnotetext{
${ }^{2}$ Areal: surface area normalized density of a metric.
} 
tion derived activated carbon (AC) films are impractical for on-wafer deposition [10]. In addition to IC process integration, promising nanostructures are demonstrating limited electrode thickness scaling, while maintaining footprint and function, capping the total energy stored by the device. These limitations have caused researchers to investigate non-conventional IC processing techniques for depositing materials such as: electrophoretic deposition, screen printing, ink jet printing, cast coating, and electrospinning [1], [11]. These fabrication methods for EDLC electrodes approach thicker, 3D-electrodes $(>10 \mu \mathrm{m})$ at the cost of specific surface area to develop higher areal energy storage. Furthermore, a more diverse selection of materials and structures can be used, making a host of carbon materials and conducting polymers accessible for IC processing.

Table 1.1: The materials and performance metrics of thin-film microscale electrochemical capacitors. Where $\mathrm{C}_{1000} / \mathrm{C}_{\text {Initial }}$ is a brief measure of the degradation of the device after 1000 cycles.

\begin{tabular}{lccccc} 
Material & $\begin{array}{c}\text { Capacitance } \\
{\left[\mathrm{mF} \mathrm{cm}^{-2}\right]}\end{array}$ & $\begin{array}{c}\text { Potential } \\
\text { Window }[\mathrm{V}]\end{array}$ & $\begin{array}{c}\text { Energy } \\
{\left[\mathrm{mJ} \mathrm{cm}^{-2}\right]}\end{array}$ & $\begin{array}{c}\mathrm{C}_{1000} \\
\mathrm{C}_{\text {Initial }}\end{array}$ & Ref \\
\hline \hline Graphene & 16 & 1 & 2 & $\sim 98$ & 12 \\
\hline Graphene & 6 & 1 & - & - & 13 \\
\hline PEDOT & 9 & 0.8 & 3 & $\sim 90$ & 14 \\
\hline CNT & $<1$ & 0.9 & $<1$ & $\sim 100$ & 15 \\
\hline Graphene/PANI & 326 & 1 & 41 & $\sim 92$ & 16 \\
\hline Graphite/Pt & 5.4 & 1 & 2.7 & - & 17 \\
\hline rGO & 0.4 & 0.8 & 0.144 & - & 18 \\
\hline
\end{tabular}

\subsubsection{Electrospun Electrodes}

Electrospinning is a technique which has attracted attention from diverse fields such as drug-delivery, tissue engineering, sensing materials, and solar cells [11. In short, electrospinning is a low cost method to deposit viscous polymer solutions as micro to nanoscale fibre mats. The ES material can incorporate additional polymeric and inorganic components to tune or enhance function. ES materials are ultrahigh surface area and can be deposited onto any grounded substrate, liquid, or simply be freestanding. The high versatility of electrospinning is sobered by the poor and primarily qualitative understanding of the process. 
ES electrodes are a large subset of electrospinning work but have not been widely used for microscale energy storage research. The comparatively large deposition abilities of electrospinning has created a focus on macroscale electrodes in devices such as wearable electronics, conductive fabrics, and filter membranes [19], [20]. In order to make high conductivity electrodes, the electrospinning community has been developing methods to deposit metal oxide, metal nitrides, organic polymers, and nanocomposites. Metal containing derivatives are often the most conductive options, but are limited by difficulties in the electrospinning process, contain or use hazardous materials, and are often mechanically fragile. Polymeric ES electrodes face the fewest ES processing hurdles and offer durable conductive electrodes. Of these materials, a popular trend in creating highly conductive ES electrodes is the high temperature $\left(700-1400^{\circ} \mathrm{C}\right)$ carbonization of ES polymers like poly(acrylonitrile) (PAN), or poly(amide-imide) (PAI) through pyrolysis $3[21-[23$. Carbonized electrodes can provide leading conductivity and consistency in ES electrodes but the fragile electrodes present an expensive burden to the thermal processing budget of a late stage IC. The most consistent electrospinning is achieved with purely polymeric solutions, where the addition of conducting polymers allows for the creation of conductive nanofibres 24]. Electrospun PEDOT:PSS has been achieved for the creation of conductive films [20], [24, [25]. Some of the applications of ES PEDOT have seen: a transparent electrode for solar cells with a minimum resistance of $12 \mathrm{k} \Omega$ 4 [25]; and a strain sensor with a peak conductivity of $1.7 \times 10^{-5} \mathrm{~S} \mathrm{~cm}^{-1}$ 24]. An ES electrode have minimal imposition on the IC processing; finding an electrospinning strategy with a selection of materials, additives, and compatible post-processing of electrodes is paramount for the process to be considered within the application.

\subsection{Thesis Contributions}

The research presented within this thesis utilizes the topics described in the aforementioned background to expand on existing ES electrode research. The work accomplished establishing a concise electrospinning method to deposit PEDOT:PSS electrodes which offer similar energy storage metrics to significantly more mature technologies, while exploring a wide variety of analysis techniques.

\footnotetext{
${ }^{3}$ Pyrolysis: The thermal decomposition of a material. This is the process responsible for the creation of charcoal.

${ }^{4}$ The $\Omega$ /sq or $\Omega$ per length, area, or volume is not explicitly stated
} 
The work begins by presenting a generic troubleshooting method for electrospinning which results in a simple ES compatible conducting polymer solution using a highly commercialized, non-toxic polymer constituents, PEO and PEDOT:PSS. The resultant ES process provides a consistent deposition of conductive ES fibres. Research for on-silicon ES for supercapacitors is sparse and this work aimed to explore the viability of the alternative fabrication method for microsupercapacitors. This thesis also presents independent confirmation of PEO/PVA/PEDOT:PSS ES solutions as a valuable independent replication study, while also building on the results to incorporate UHMW PEO as a stable carrier polymer. With SEM images captured of different and novel fibres, the work also explores the use of multiple techniques to conduct analysis while testing leading equivalent circuit models and data fitting methods. These electrodes aim to demonstrate the sparse porosity, with respect to leading microscale EDLC electrodes, of ES mats could reduce thickness scaling problems seen in leading microscale EDLC electrodes. The work presented in chapters 3 and 5 has resulted in a talk given at the Materials Research Society's MRS 2018 conference:

- C. Fernandes, L. MacEachern, and S. McGarry, "Electrospinning supercapacitors for microelectronic energy storage", in Materials Research Society 2018. MRS, 2018. [Online]. Available: https://mrsspring2018.zerista.com/event/member/466308.

Followed by the current compilation of a paper targeted for ACS Materials \& Interfaces for a publication encompassing the electronspinning of the final device and results of chapter 3.5 and 6 . 


\section{Chapter 2}

\section{Background}

\subsection{Principles of Polymers}

The invention of the first synthetic polymer in 1907 allowed for a world of new materials. These chain-like molecules soon dominated a wide variety of applications due to their customizable physical and chemical properties, which arise from complex intermolecular and intramolecular effects. This section will outline some of the introductory principles of polymer science within the scope of this work. First, the structures of polymers, critical properties and behaviors in solution. Secondly, properties of PEDOT:PSS, a conducting polymer (CP), will be introduced and described.

\subsubsection{Polymer Structure}

Polymers are long chain molecules of repeated unit molecules known as monomers. The properties of a polymer material are primarily dependent on two aspects: first, the structure and chemical properties on the monomer unit; second, the number of monomer units bonded together (the length of the polymer chain), also referred to as the degree of polymerization (can often be as high as $\left.10^{6}\right)$. For example, simple monomer units such as ethlyene oxide and vinyl alcohol, seen in figure 2.1. consist of a carbon backbone with an oxygen substituting every third carbon. When bonded together end-to-end these form poly(ethlyene oxide) and poly(vinyl alcohol), respectively. These simple structure allows for polymerization of extremely long chains. For poly(ethlyene oxide), the lack of both a localized positive region and a rigid backbone makes this polymer soft and smooth, leaving it for use in makeup, food additives, and pharmaceuticals [26]. 

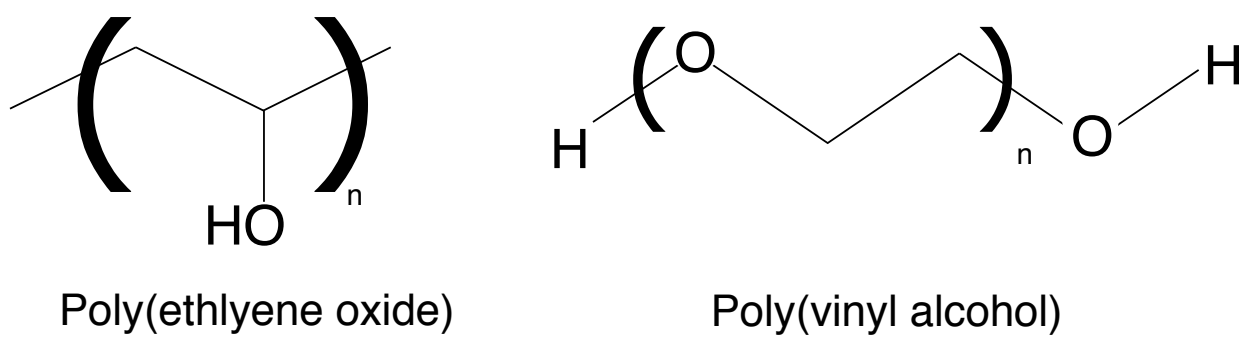

Poly(vinyl alcohol)

Figure 2.1: The monomer units of two common polymers: poly(ethlyene oxide) and poly(vinyl alcohol).

In order to simplify these complex polymer systems, they can be broken down into short-range interactions: bond stiffness and angles of rotation; and long-range interactions: intermolecular forces (hydrogen bonding, polarity effects). First, we begin with a single linear polymer chain dissolved in solution. A free floating polymer will bend or curl, with these shapes called the polymer's conformation 26.

For different polymers, their conformation will be dependent on the number and type of monomer units. The thermodynamically probable conformations can be thought of as a sphere gyrating about a center of mass. The mean square distance between each monomer unit and the conformation's center of mass provides the analytically useful radius of gyration, $R_{g}$, of this sphere. $R_{g}$ of a simple uniform polymer chain is calculated through 26$]$ :

$$
R_{g}^{2}=\left\langle\frac{1}{(N+1)^{2}} \sum_{i, j=0}^{N}\left(r_{i}-r_{j}\right)^{2}\right\rangle \quad \text { (Valid for any conformation) }
$$

Where $\mathrm{N}$ is the degree of polymerization and $r_{i}-r_{j}$ is the distance between any two monomers. Finding the exact position of every monomer unit in solution is impractical, therefore polymer scientists make use of $\nu$, the Flory exponent. The Flory exponent is dependent on the distribution of the polymer, and is often treated as 1 for ideal polymers and $\approx \frac{3}{5}$ for real chains. As complex long-range interactions and the solvent's ability to fully solvate the polymer molecules are taken into account, the Flory exponent's value will vary but is bounded at the theoretical maximum of 1 . $R_{\mathrm{g}}^{2}$ 
can now be expressed as a function of the size and number of the monomers, $\mathrm{b}$ and $\mathrm{N}$ respectively:

$$
R_{g}=b N^{\nu}
$$

By extending this, we can look at a crude approximation of the monomer volume fraction as the ratio of absolute volume of a polymer, $\mathrm{Nb}^{3}$, over the radius of gyration:

$$
\frac{N b^{3}}{R_{g}^{3}}=\frac{N b^{3}}{\left(N^{\nu} b^{3}\right)^{3}} \approx N^{1-3 \nu}
$$

The volume fraction is inversely proportional to the degree of polymerization as $3 \nu \geq 1$. As polymer concentration increases, these spheres begin to entangle with one another and can overlap due to a lack of free space within the solution, denoted by $c_{\text {entanglement }}$. This can be qualitatively thought as any single point within a solution is connected to the rest of the solution through the entanglement points thus pulling on one polymer is pulling on all the solvent molecules of the long chain and all chains they are entangled as well, giving rise to a sharp increase in viscosity [26]. Since the radius of gyration is difficult to determine experimentally, Equation 2.2, the Mark-Houwink Equation, is an experimental relationship between the intrinsic viscosity, $[\eta]$, and molecular weight, $\mathrm{M}$, aiding for approximate values of intrinsic viscosity when the system consists of a simple polymer and a solvent.

$$
[\eta]=\kappa M^{a}
$$

With $\kappa$ and a being the Mark-Houwink constants determined experimentally for different polymer/solvent combination. With further increasing concentration, a solution becomes semidilute, $\mathrm{c}^{*}$, beginning at $c * \geq 10 \times c_{\text {entanglement }}$ where that can be related back to Equation 2.2 by $[26$ :

$$
c^{*}[\eta]=1
$$


Combining equations 2.2 and 2.1.1 provides an approximate entanglement concentration for different polymers and different molecular weights:

$$
c_{\text {entanglement }}=\frac{10}{\kappa M^{a}}
$$

Equation 2.3 demonstrates that entanglement can occur at extremely low concentrations when the molecular weight of a polymer is high as $10^{6}$.

\subsection{PEDOT:PSS : A Conducting Polymer}

\subsubsection{Introduction}

As the understanding of polymer chemistry advanced, more complex monomers and polymer systems allowed for the creation of materials with a wide variety of traits and characteristics. By the late 1970s, a Nobel Prize winning ${ }^{1}$ research demonstrated that some polymers could have their electrical resistivity reduced into a conductive state. Since then, work on intrinsically conducting polymers (CPs) has grown and diversified [27]. Three conducting polymers of major interest are: polypyrrole (PPy), poly(aniline) (PANI), and poly(thiophene) (PTh). These polymers dominate the vast majority of research as they all have good conductivity and environmental stability. However, these polymers suffer from either processability, mechanical fragility, or have limited biocompadibility due to toxic byproducts during degradation. An incredibly interesting derivative of PTh is poly(3,4-ethylenedioxythiophene) (PEDOT). Compared to PTh, PEDOT possesses a dioxyalkylene group, which improves conductivity and enhances electrical, thermal, and chemical stability [28], [29]. PEDOT alone suffers from poor processability. However, it is commonly used within a complex with water soluble PSS. The added solubility allows PEDOT:PSS to be easily incorporated into inks and solutions to be ink-jet printed, spin cast, and screen printed. Today PEDOT:PSS has become widely adopted in organic field-effect transistor (OFET), electrochromic, bioelectronic, carrier transport, and anti-static applications 28.

\footnotetext{
${ }^{1}$ Awarded in 2000 to Alan J Heeger, Alan G MacDiarmid, and Hideki Shirakawa
} 


\subsubsection{Structure of PEDOT:PSS}

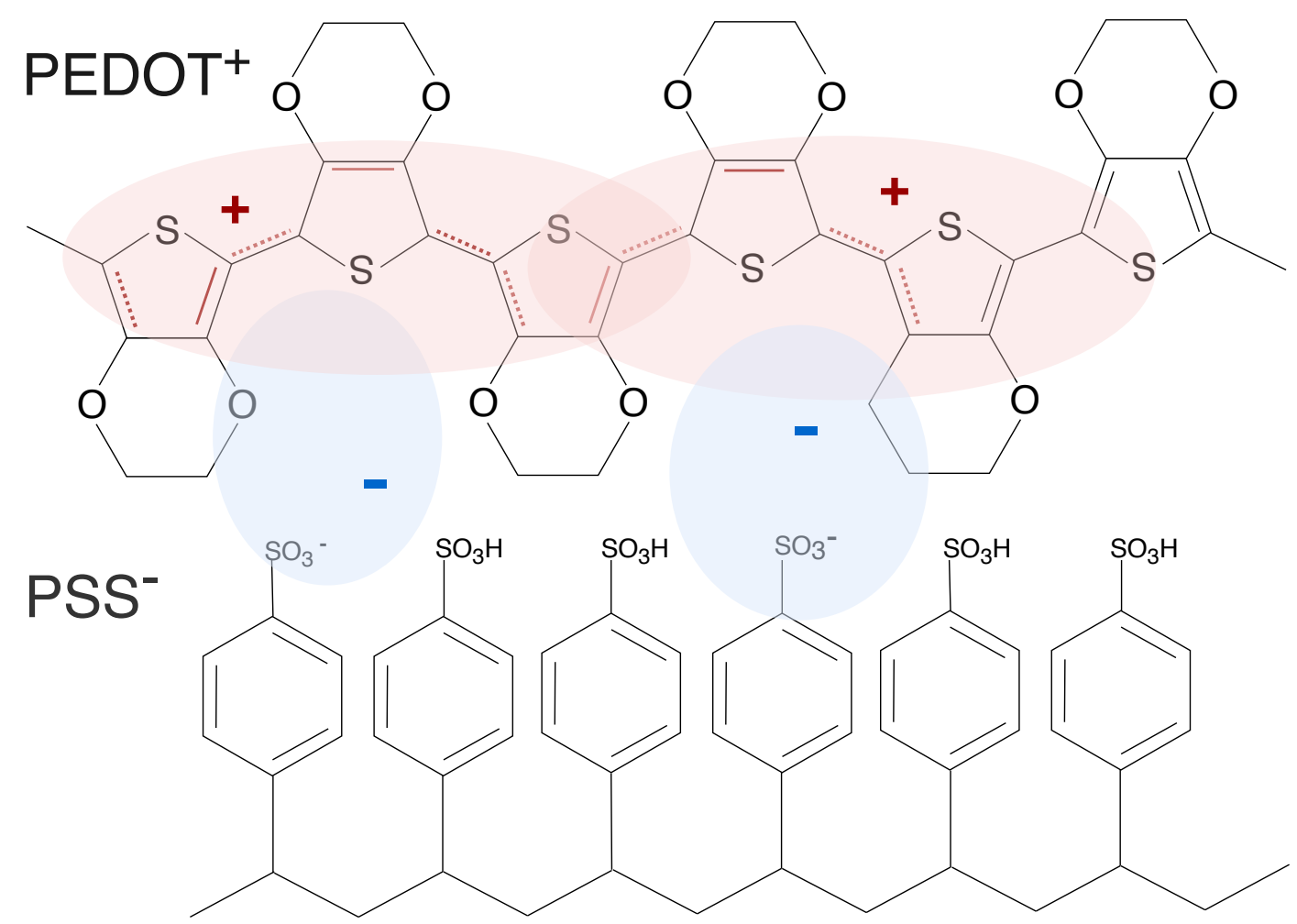

Figure 2.2: The monomer units of PEDOT:PSS illustrating the creation of a hole conducting $\pi$-conjugated backbone, shown in red, due to PSS's acceptance of PEDOT's delocalized $\pi$-electron.

PEDOT:PSS is a clever complex making the most of each component's properties. PEDOT (and other conducting polymers) have alternating single and double bonds along the carbon (thiophene rings) backbone. A conjugated backbone allows for the delocalization of an electron and movement of a hole charge species throughout this backbone. This electron is the $\pi$-electron $2^{2}[28$. The Bayer AG research laboratories paired the loosely bound $\pi$-electron on the ethylenedioxythiophene (EDOT) monomer with the oxidative properties of PSS. The PSS accepts the PEDOT $\pi$-electron allowing for the movement of charge throughout the PEDOT backbone. In the conducting polymer field, PSS would be considered the dopant to PEDOT, as it creates mobile charge (new electronic states) $3^{3}$ This doping allows for hole-transport through the conjugate carbon backbone, which is the accepted mechanism for PEDOT:PSS's conductivity. Figure 2.2 illustrates the hole mobility

\footnotetext{
${ }^{2}$ The $\pi$-electron is named after the one of the double bond components as this atomic orbital overlaps down the conjugated backbone allowing for conductivity 28

${ }^{3}$ More pedantically this is an oxidation reaction of the PEDOT and not doping in the semiconductor sense but both do allow for the creation of mobile charge by generating additional electronic states.
} 
through the conjugated backbone of PEDOT shown as the dashed red bonds and the localized reduction of PSS due to acceptance of a $\pi$-electron [27], [28]. PSS's most important contribution to the complex is water solubility allowing for use in a wider variety of deposition methods 4

\subsubsection{Solvent Conductivity Effects}

The details surrounding the specifics of the charge motion mechanisms in PEDOT:PSS is not well understood [28]. Despite theoretical gaps it is known that a temperature curing post deposition and the addition of polar solvents to a PEDOT:PSS dispersion can drastically increase the conductivity of the subsequent deposition, termed conductivity enhancement agent (CEA) (also called secondary dopant:5 [28], 30], 31]. The CEA does not remain in the dried film after deposition unlike a CP dopant (such as PSS). Thin film cured PEDOT:PSS demonstrates that the constituents arrange into continuous regions of PEDOT and PSS allowing for conductivity purely through the PEDOT, this is illustrated by Figure 2.3. The quality of this conformation leads to the observed conductivity of the material. Polar solvents act as CEAs for PEDOT:PSS by causing an improvement in this organization of the PEDOT:PSS's once dried, often in elevated temperatures [30. The resulting bulk conformation change in PEDOT:PSS films is suggested to lead to better bulk conduction through the film.

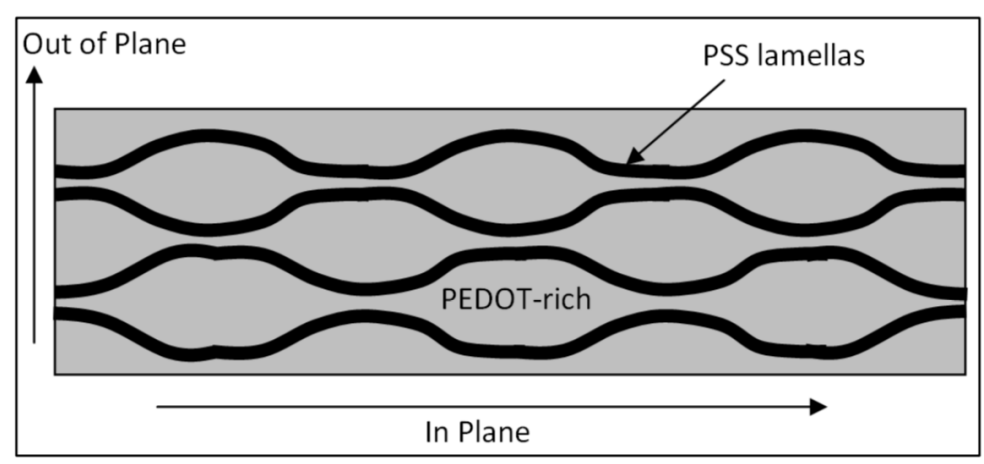

Figure 2.3: PEDOT:PSS idealized thin film conformation diagram. Illustrating the conduction regions of PEDOT layers forming within a PSS bulk 32$]$.

\footnotetext{
${ }^{4}$ PEDOT:PSS maintains PSS's water solubility but PEDOT:PSS inks contain a host of added solvents, surfactants, and/or stabilizers in order to prevent clumping and non-uniform dispersion.

${ }^{5}$ Once again the term dopant is used but these agents do not create electronic states, nor remain in the material.
} 
A few CEAs often used on PEDOT:PSS are tetrahydrofuran (THF), dimethylformamide (DMF), and dimethyl sulfoxide (DMSO). All of these are highly polar solvents with a range of other solvent properties such as vapor pressure, proticity, and relative permittivity $\left(\epsilon_{r}\right)$. Of these, DMSO has been shown to invoke the largest conductivity increase in PEDOT:PSS [30], [31].

\subsection{Electrospinning}

\subsubsection{Introduction}

Electrospinning has demonstrated an ability to be an inexpensive method to deposit micro to nanoscale fibres onto a variety of conductive substrates or as a freestanding film. Despite the early observation of the process in the early 1900's, electrospinning's research and development within the field of electronics is modern [11], [33. The deposition is a non-woven mat of fibres resembling a fabric. This section will outline control parameters of electrospinning.

\subsubsection{The Principles of Electrospinning}

To obtain the electrospinning phenomenon, a simple apparatus uses: a blunt-tip needle filled with the solution for deposition, a collection substrate, and a high applied potential difference between the solution and the collection substrate. Figure 2.4 illustrates this example apparatus and the distinct regions (A through $\mathrm{D}$ ) of the ES process as the polymer solution is expelled from the needle tip and is stretched and dried into a microscale or nanoscale fibre on its journey to the collector. 


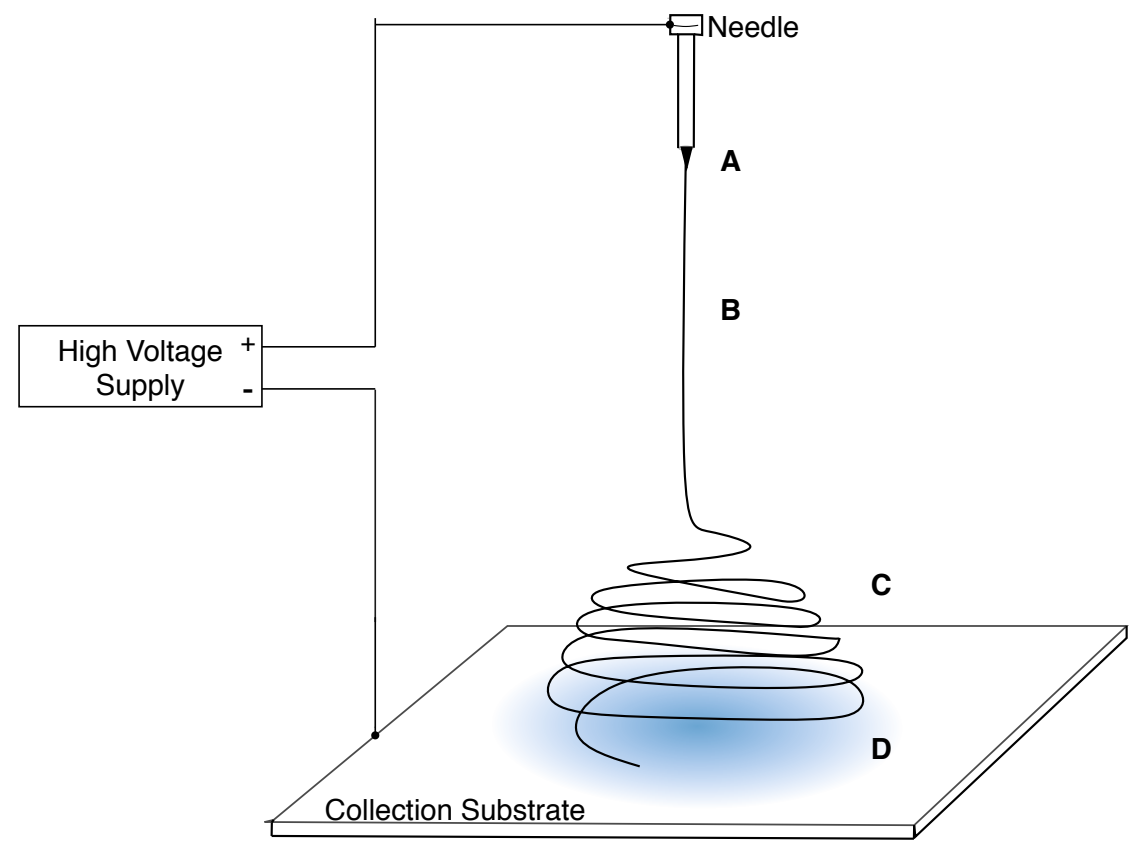

Figure 2.4: Illustration of a simple ES apparatus using a high voltage differential between a solution filled needle and a collection substrate

Region A: Taylor Cone: Electrospinning relies on the high voltage formation of the Taylor Cone. First characterized by Sir Geoffry Taylor in $1964[34]$. The Taylor Cone is the charged solution building of charge and repelling itself forming a conical structure. With sufficiently applied voltage, the local high field point at the tip of the Taylor Cone allows the polymer solution to overcome the surface tension and eject from the droplet in a continuous stream of a diameter significantly smaller than that of the needle [35].

Region B: Stable Region: With a steady stream of solution being ejected from the high field point. The solvent begins to quickly evaporate as this relatively thin stream is rapidly accelerated away from the needle. The evaporation causes the stream to continue to thin causing an increase in surface charge density. A leading theory suggests that at a critical point, this increasing surface charge causes the stream to become unsteady and begin to rotate and whip [33].

Region C: Whipping Phenomenon: This is a not well understood region of operation as the fibres begin to move incredibly fast while continuing to stretch and thin even further, while also not remaining very localized. Imaging, observation and quantitative description of the whipping phenomenon remains incomplete [33], [35]. This whipping is suggested to stretch and thin the fibre but can also cause the fibre to fracture under the intense Coulomb force [35]. 
Region D: Collection: The whipping phenomenon continues until the fibre reaches the collection plate to deposit the now densely accumulated charge. The resultant deposition is an often uninterrupted single fibre orders of magnitude thinner than the needle it is ejected from. These fibres rapidly trace over the collector and have shown to be able to build freestanding webs as material builds up [33].

\subsubsection{Electrospinning Parameters}

The electrospinning process exists near electrodropleting, another processes driven by high voltage solutions [33], [35]. Where the type, molecular weight, and concentration of the constituents within the solution and the applied voltage, spinning distance, humidity and temperature influence whether electrospinning or electrodropleting will occur. When these variables are correctly balanced, electrospinning is obtained. Due to the poorly understood nature of electrospinning, much of the existing literature is a qualitative guide to obtaining electrospinning. The different influences on electrospinning can be collected into two distinct categories: apparatus parameters and solution parameters. The following sections will present short summaries of widely accepted parameters and their accepted influence on the electrospinning process.

\section{Apparatus Parameters}

Spinning Distance: The distance between the needle and the collector dictates the electric field strength (At a specific applied voltage) acting on the solution and the space in which the fibre has to dry and electrospin before being collected. A short distance can allow spinning at lower voltages but may not allow for enough time for the drying to occur. This parameter is closely related to the humidity, applied voltage, and viscosity.

Humidity: The humidity in the chamber will influence drying rate of the fibre. A slower drying time has demonstrated increase uniformity of fibres.

Applied Voltage: A minimum voltage is required to overcome the surface tension of the solution to achieve electrospinning. The applied voltage is also limited by electrospraying, a process when the electric field strength rips the solution's charged jet stream into microdropplets. 


\section{Solution Parameters}

Viscosity: The viscosity of the fluid is closely related to the fluids ability to form the Taylor cone during high voltage charging. The viscosity will influence the required voltage and spinning distance for electrospinning. The deformation force on the solution will also influence the solutions thinning during whipping.

Molecular Weight: The molecular weight of the polymer constituents will dictate entanglement in the solution. This will govern the surface tension and the viscosity. Both of these are critical parameters tied to the applied voltage and spinning distance. A high molecular weight will allow for spinning at lower solids concentrations but will require a longer spinning distance, lower humidity, or high vapour pressure solvents to allow the fibre to dry before reaching the collector.

Solvent and Additives: Solvents and additives allow for different radii of gyration of polymers influencing the entanglement and in turn the molecular weight. Solvents will have a varying surface tension, vapour pressure and dielectric constant influencing the drying rate, the solutions interaction with the applied voltage, and the morphology of the fibre.

\subsection{Electrolytic Capacitors}

\subsubsection{Introduction}

The simplest capacitor is a parallel-plate consisting of two electrodes separated by a dielectric separator. An electrolytic capacitor is simply replacing the dielectric with an electrolyte. The applied potential now perturbs the contained electrolyte storing the energy in the electrolyte ions gathered at the surface of the electrode, or in redox reactions occurring within the interface. Unlike conventional parallel-plate capacitors where the high mobility of charge carriers allows for apparent fast motion of charge, electrolytic capacitors are kinetically mediated by diffusion, adsorption, and potential dependent reduction-oxidation reactions [36], [37]. The dominate effects present in an electrolytic capacitor are separated into the electrode-electrolyte region and effects within the bulk solution between the electrodes. 


\subsubsection{Electrode-Electrolyte Processes}

\section{The Electrolytic Double Layer}

When an electrolyte is in contact with a conductor, the free moving ions can be balanced by the free moving charge within the conductor, creating the electrolytic double layer (EDL). With an applied potential, the moving ionic charges balance the electrode potentials. This build up of ions, the EDL, was described by Hermann von Helmholtz in 1853 analogously to a parallel-plate capacitor termed the Helmholtz capacitance $C_{H}$ by 38 :

$$
C_{H}=A \frac{\epsilon}{d}
$$

Where $\mathrm{A}$ is the area of the electrode/electrolyte interface, $\mathrm{d}$ is the approximate radius of an ion and/or solvent molecules collecting at the interface, and $\epsilon=\epsilon_{0} \epsilon_{r}$ is the permitivitty of the solvated ion complex. Here we see the key mechanism that gives rise to EDLC's high capacitance as now the nanostructuring or porosity of an electrode can increase $\mathrm{C}$ without needing more footprint and the atomic scale of the separation distance between the electrode and ion. Unfortunately, this rigid parallel layer is an oversimplification as not all charge can approach that close Figure 2.5 is an effective description presented by Graham that combines three theories and views the EDL in 3 regions: Inner Helmholtz Plane (IHP), Outer Helmholtz Plane (OHP), and the Diffusion layer [37- 40 .

Figure 2.5 illustrates Helmholtz' original description of the EDL. By splitting the Helmholtz layer into two distinct layers. These two layers make up the Stern model which accounts for the ions to be specifically adsorbed onto the electrode interface termed the inner Helmholtz plane, and the electrostatically held outer Helmholtz plane $[37]-[39]$. These adsorbed ions can partake in faradaic processes such as redox reactions, adding to capacitance but are slow in comparison to electrostatic EDL formation. The Stern model assumes that the formation of the layers are potential independent but are limited by the volume of the ions. 


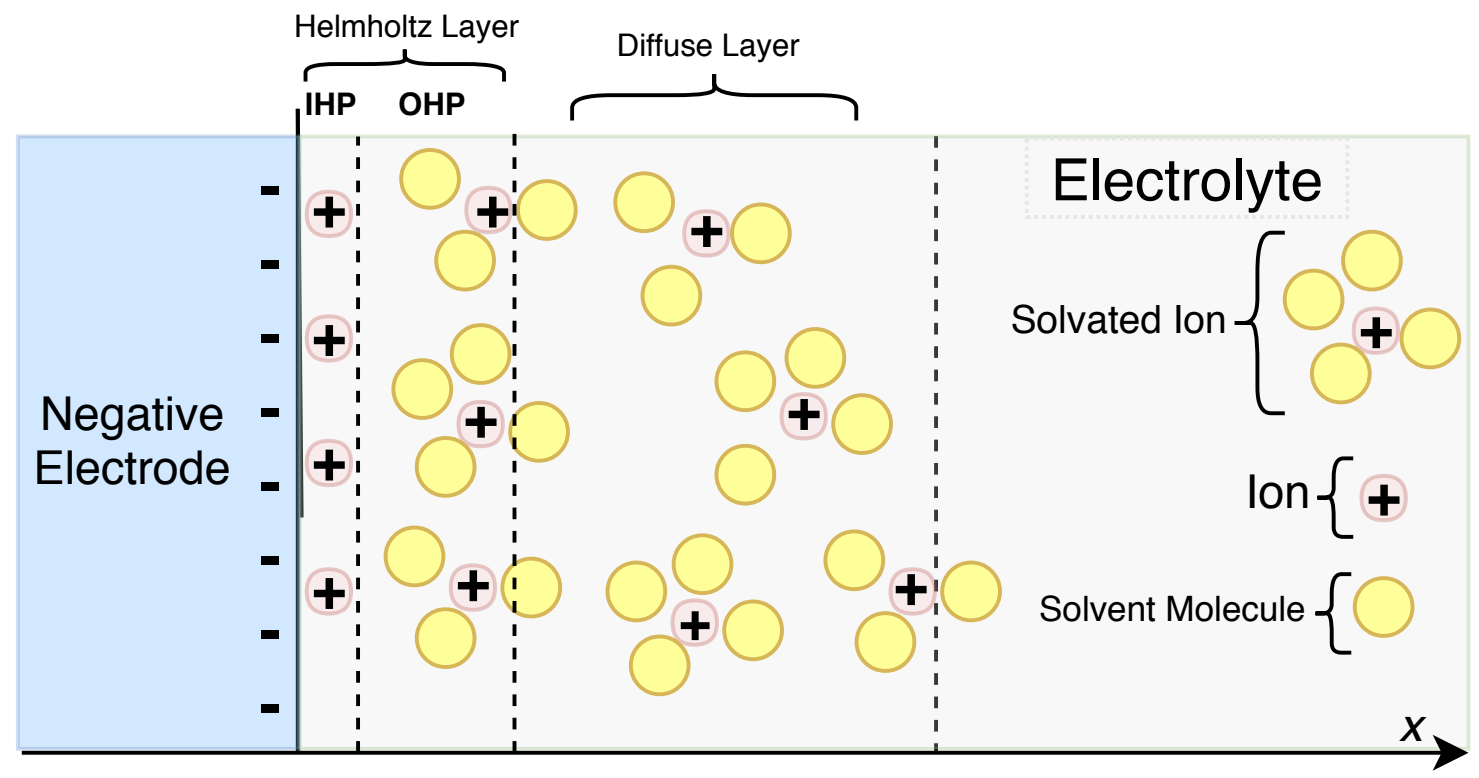

Figure 2.5: The different layers of the EDL, where the IHP is directly at the electrode interface forcing other ions away. The solvent molecules here are arranged and sized arbitrarily but often solvent molecules, specifically organic solvents, are larger than that of the ions.

The final component of the EDL is the diffuse layer existing after the OHP. First proposed as an independent description of the electrolytic double layer, Guoy-Chapman treated this layer as a kinetically limited diffusion process described by a Boltzmann distribution. This allowed for the charge to be more distributed, creating a thicker EDL due to the characteristic long Boltzmann tail giving rise to a capacitance described by [37, [39]:

$$
q_{\mathrm{diff}}=\sqrt{\frac{2 k T n_{0} \epsilon_{0} \epsilon_{r}}{\pi}} \sinh \frac{e_{0} V}{2 k T}
$$

Where $k$ is Boltzmann's constant and $T$ is temperature. Graham built on both Helmholtz and Guoy-Chapman by describing the diffuse layer as an additional region to the Stern layers. This required a correction to Equation 2.5 to account for shielded and more distant potential seen by the ions within the diffuse layer 38, 39]:

$$
q_{\text {diff }}=\int_{0}^{\infty} \frac{\epsilon_{r} \epsilon_{0}}{\lambda_{\text {DEBYE }}} \cosh \frac{z_{i} e_{0} \phi_{D}}{K_{B} T} d \phi_{D}
$$

Where the thickness of the diffuse layer known as the Debye length, labelled $\lambda_{\text {DEBYE}}$. The Debye 
length is a measure of length which accounts for electrostatic screening. This value is related to the length it takes for ions in the solution to collect and screen an electrostatic potential.

The capacitive contributions from the diffuse layer are often dwarfed by the Stern layer as a Helmholtz layer can be on the order of 1-2 nm, whereas the diffuse layer can be up to $1000 \mathrm{~nm}$ [38]. In a pure EDLC, the IHP and OHP are the dominant capacitive contributions. In an electrochemical device with non-inert electrodes, such as organic polymer or metal oxide electrodes, the dominate energy storage can come from chemical reactions as well as the EDL, these are the capacitive effects within pseudocapacitors.

\section{Pseudocapacitance: Electrochemical Charge-Transfer}

Electrochemical charge-transfer processes are slow $\left(10^{0}-10^{-6} \mathrm{~Hz}\right)$ Faradaic processes in which electric charge moves between the electrode and electrolyte. This variety of chemical reactions are mechanisms in pseudocapacitance which dominate charge storage in pseudocapacitors and chemical batteries, where an in-depth description can be found here [38. The crude description can be given as a DC potential dependent resistance, $\mathrm{R}_{\mathrm{CT}}$ and capacitive energy storage, $C_{O X}$ and $C_{R E D}$. The latter are the capacitive energy storage of the reduction and oxidation reactions. Not all these electrochemical reactions are reversible and lead to a reduction in performance. The reactions occurring are dependent on the magnitude of the applied potential, electrode/electrolyte material, temperature, and more [39]. These reactions are the primary cause for the aging, loss of storage and slow charging rates of certain energy storage devices.

\subsubsection{Electrolyte Processes}

\section{Diffusion Impedance}

Diffusion processes are governed by mass-transport occurring in the $10^{2}-10^{-1} \mathrm{~Hz}$ frequency range. In electrochemical capacitors, the ion motion in the bulk electrolyte is due to concentration gradients created by the adsorption and consumption of charge at the interface region in an electrochemical

\footnotetext{
${ }^{6}$ This is dependent on the the availability of electrolyte ions. As electrolyte concentration increases the diffuse layer decreases in thickness.
} 
system (ECS) [37], [38]. A concentration driven potential gradient is used in conjunction with the impedance created by electroactive ion current flow through the bulk, termed the Warburg impedance. This impedance is AC and DC dependent and comes in two main flavors: semi-infinite or finite depending on the availability of electrolyte ions and the thickness of the bulk region, compared to the thickness of the diffusion layer, $\delta$, in the interface region [38]:

$$
\begin{gathered}
\text { Semi - infinite : } \quad Z_{W}=\frac{1}{Q_{W} \sqrt{j \omega}} \cosh \left[\frac{\delta}{\sqrt{D}} \sqrt{j \omega}\right] \\
\text { Finite : } \quad Z_{W}=\frac{1}{Q_{W} \sqrt{j \omega}} \operatorname{coth}\left[\frac{\delta}{\sqrt{D}} \sqrt{j \omega}\right]
\end{gathered}
$$

Where $Q_{W}$ is given by:

$$
Q_{W}=\frac{4 R_{G} T}{z^{2} F^{2} A C * \sqrt{2 D}}
$$

This relationship is extremely complex relying on: $R_{G}$, the ideal gas constant; $T$, temperature; $F$, the Faraday constant; $z$, transferred electron charge from diffusion species; $D$, diffusion coefficient; $A$, surface area; and $C$, concentration. Despite its complexity the Warburg impedance is extremely valuable for qualitative analysis within an ECS as it is associated with a slanted flat region 7 in which both real and imaginary impedance increase in unison. This allows for the easy identification of the Warburg impedance dominating over other phenomenon at specific frequencies.

\section{Bulk Electrolyte Impedance}

Within a device, often the thickness of an EDL is much less than the space between electrodes, the bulk media or electrolyte. This bulk media consists of mobile ions and insulting (solvent) background but to investigate the resistance caused by the bulk it is useful to treat the electrolyte whole as a homogeneous solution [39]. During operation or testing, the ion migration is driven by the applied electric field and the electrolyte can conduct charge between the electrodes. Migration-

\footnotetext{
${ }^{7}$ When the Warburg impedance dominates the $\sqrt{j \omega}$ will equivalently distribute impedance into both domains.
} 
driven ionic conduction can be represented as a function of the bulk concentration of ions, $C^{*}$, and their respective mobility and charge [38].

$$
R_{B u l k}=\frac{d_{B u l k}}{A T} \frac{1}{\sum_{i}^{N} z_{i} \mu_{i} C_{i}^{*}}
$$

Where $d_{B u l k}$ is the thickness of the bulk, $A$ is the cross section of the current flow path, and $T$ is temperature. Grouping the bulk homogeneous resistivity as $\rho . R_{B u l k}$ can be simplified into:

$$
R_{\text {Bulk }}=\rho \frac{d_{b u l k}}{\mathrm{~A}}
$$

The solvent and ion constituents of an electrolyte act as suspended polarized particles when exposed to an AC current characteristics of dielectric materials. Cole and Cole described a frequency dependent dielectric constant to account for frequency dispersion in dielectric media [38]. These mid-to-low frequency dependent phenomenon cause small polarization capacitance which induces a shifting capacitance at the interface, the local polarization, eliciting a larger voltage drop through the bulk. This can cause better isolation between electrodes as this raises the resistance seen by an applied DC voltage.

\subsubsection{Summary}

An electrochemical capacitor is an extremely complex device where a broad overview of the present dominant phenomenon helps understand the design choices to be made when operating. The main factors contributing to the energy storage within the device are the creation of the EDL and chargetransfer redox reactions at the electrode/electrolyte interface. The EDL is a fast forming reversible process and is dependent on electrolyte ion size, electric field strength, and surface area. Redox reactions are slower, can be non-reversible, and are potential mediated. Thus the selection of the electrode material, operation regions and the electrolyte will all impact the device parameters. 


\subsection{Electrochemical Capacitor Investigation Techniques}

\subsubsection{Introduction}

The study of electrochemical capacitors requires a range of techniques to discern the specific mechanisms occurring during operation. In order to understand the function of an electrochemical capacitor, three major techniques are used. Each technique looks at specific aspects of the device in different depths:

- Charge-Discharge (CDC): This provides top level device functionality characterization: how much energy can be stored by the device? Conducted by applying a DC voltage step voltage or current, and measuring the time domain current or voltage responses respectively. The limit in energy storage of the device can be distilled [40]. DC charging investigation does not easily provide insight to the specific mechanisms occurring within a device.

- Cyclic Voltammetry (CV): Provides low frequency insight to voltage dependent processes. For CV, a voltage ramp is cycled multiple times as the current response is measured. As mentioned before, electrochemical capacitors can store energy within the electrolytic double layer or within charge stored within potential dependent redox reactions 40, 41]. CV has characteristic curves and shapes which differentiate between non-faradaic, and faradaic electrochemical energy storage.

- Electrochemical Impedance Spectroscopy (EIS): The most phenomenologically in-depth analysis conducted within this body of work. A small $\mathrm{AC}$ voltage is applied to the device and the frequency is swept through an extremely wide spectrum as the complex impedance is measured [38], [39]. EIS can then be used to qualitatively and quantitatively characterize the different capacitive and resistive mechanisms outlined Section 2.4 .

The methods and governing equations for analysis will be outlined in the sections below. 


\subsubsection{Charging}

Two primary methods for DC testing of a device are galvanostatic or potentiostatic testing, where DC current or voltage is applied to a device under test (DUT) measuring the time-dependent voltage or current response, respectively. From here, for an ideal capacitor, C, with a resistor, R, in series we begin with a universal time-constant equation 40]:

$$
V_{f}=V_{S}\left(1-e^{-\frac{t}{R C}}\right)
$$

Where $\mathrm{RC}=\tau$ is the time-constant, $\mathrm{V}_{\mathrm{f}}$ is the final voltage after $\mathrm{t}$ seconds. Equation 2.11 governs both the current and voltage charging and discharging and can be related by Ohm's Law. Another approach to capacitance, when pseudocapacitance and other phenomenon cause deviation from the time-constant model can be seen in Equation 2.12

$$
C=\frac{I_{D C}}{\frac{d V}{d t}}
$$

Giving the energy stored as:

$$
E=\frac{C V^{2}}{2}
$$

Here $I_{D C}$ is an applied DC step current and $\frac{d V}{d t}$ is the slope of the measured voltage characteristic. Unfortunately, in electrochemical devices $\frac{d V}{d t}$ is non-constant and these simple electrostatic equations fail to capture the complexity of EDLC. More complex models are available but this body of work aims to investigate the complexity through the later techniques within the chapter.

An applied DC voltage while measuring the current flowing into the DUT provides a raw look at the charge being stored by integrating the current-time curve produced, giving the total charged stored by:

$$
q=\int_{t=0}^{t_{\max }} I(t) d t
$$


Since we understand that an electrochemical capacitor will not be electrostatically governed like a parallel-plate capacitor, a numerical integration can avoid over simplification giving the energy stored to be:

$$
E=\frac{V}{2} \int_{t=0}^{t_{\max }} I(t) d t
$$

\subsubsection{Cyclic Voltammetry}

CV analysis requires the linear cycling of an applied voltage to a device while the resultant current is measured. The CV results of an ideal electrostatic parallel-plate capacitor produces a nearly rectangular shape whereas charge-transfer dependent processes will produce mounds or hills that signify redox-reactions occurring at that applied voltage. This behavior is motivation of the CV analysis as it provides simple visual separation between voltage dependent electrochemical capacitor phenomenon. For an ideal capacitor the non-faradaic CV curve is governed by [40]:

$$
C=\frac{1}{\Delta V \frac{d V}{d t}} \int_{V=0}^{V_{\max }} I d V
$$

Where $\Delta V=V_{\text {Max }}-V_{0}$ is the range of the linear voltage cycle, and $\frac{d V}{d t}$ is the linear sweep rate. Under ideal assumptions the energy stored can be found by:

$$
E=\frac{\Delta V^{2}}{2 \frac{d V}{d t}} \int_{V=0}^{V_{\max }} I d V
$$

We can see from equations 2.16 and 2.17 that the sweep rate will have an impact on the values, this is readily seen in kinetically limited devices (like ECs) where a slow sweep rate will cause deviation from the rectangular shape. The current integral seen in the CV equations can always be found numerically, which can also characterize the energy stored by pseudocapacitive redox-reactions. 


\subsection{Electrochemical Impedance Spectroscopy}

\subsubsection{Introduction}

The most powerful technique used within the scope of this work is EIS. EIS is conducted sweeping the frequency of an $\mathrm{AC}$ voltage and measuring the impedance response of a load, allowing for temporal isolation of the different capacitive and resistive mechanisms within an electrochemical system. In a linear system, an applied $\mathrm{AC}$ voltage, $V_{\text {in }}$ to a system will produce a current response, $I_{\text {out }}$, with a phase shift, $\phi$ and magnitude relating to the complex impedance, $Z$, of the system, given by the relations in Equations 2.18 .

$$
\begin{gathered}
Z=\frac{V_{\text {in }}}{I_{\text {out }}} \\
V_{\text {in }}(t)=V_{0} \sin \omega t, \quad I_{\text {out }}=I_{0} \sin (\omega t+\phi)
\end{gathered}
$$

As the radial frequency, $\omega$, is varied from millihertz to megahertz the different mechanisms which occur at different rates result in either real (resistive) or imaginary (capacitive or energy storage) impedance. For example, the ideal real resistance seen within the interconnection wires of the device will be present at all frequencies, but the EDL is limited by the ion mobility at megahertz frequencies, allowing for the separation of the impedance contributions.

EIS is powerful as it can differentiate between the effects of electrochemical kinetics at electrodeelectrolyte interface and the bulk allowing for the separation of the time-dependent phenomenon like charge-transfer and mass-transfer mechanisms [39]. EIS is also used for understanding effects about electrode porosity, material and electrolyte choice. Differentiating between and analyzing different processes is a complex endeavour that requires the selection of, sometimes non-unique, circuit models followed by complex, often non-linear, data fitting to equations with numerous variables [38]. It is established as an effective qualitative analysis with quantitative insight which needs to be used 
in conjunction with other techniques to ensure validity. This section will outline the concepts of EIS, specific data representation conventions, followed by examples of equivalent circuit models for electrochemical capacitor devices, and a brief outline of the methods and problems faced by data fitting.

\subsubsection{EIS Data Representation}

To allow for quick qualitative recognition of the electrochemical processes present in a system, researchers use Bode and Nyquist plots to display the data. For example, representing a simple EDLC with the circuit shown Figure 2.6, one can conduct a frequency sweep and collect the impedance.

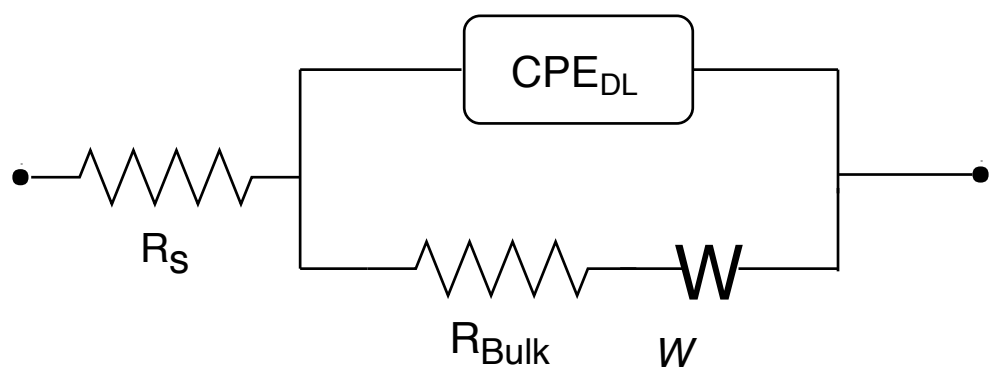

Figure 2.6: A simple equivalent circuit model which accounts for the non-ideal capacitance with a constant-phase element (CPE) and the diffusion impedance using a Warburg element (W).

Figure 2.7 shows the results of a simulated impedance sweep of the simple parallel circuit in Figure 2.6. The data is plotted with both conventions: plot A show a logarithmic frequency scale for the impedance vs. frequency, to proportionally represent the real and imaginary impedance across the large testing range. Plot B is the Nyquist plot representation of the impedance data. In EDLCs, a Nyquist plot is the $\mathrm{Z}_{\text {Real }}$ vs $-\mathrm{Z}_{\text {Imag }}{ }^{8}$ where the plot begins with the high frequency data giving low impedance. Nyquist plots allow for visual determination of different conventional electrochemical behaviours, and simple extraction of specific examples by breaking down the geometry and looking at what distinct shapes can be found. Looking at Figure 2.7B, from left to right, the nyquist plot demonstrates a parallel RC phenomenon through the semi-circular shape, followed by the mid-low frequency Warburg (W) component dominating displaying its characteristic $45^{\circ}$ linear region before an asymptote-like low frequency region. We see that the resistance of different phenomenons can be extracted.

\footnotetext{
${ }^{8}$ The negative of the imaginary component of the impedance is often used in the study of electrochemical capacitors as all the processes produce purely capacitive impedance.
} 

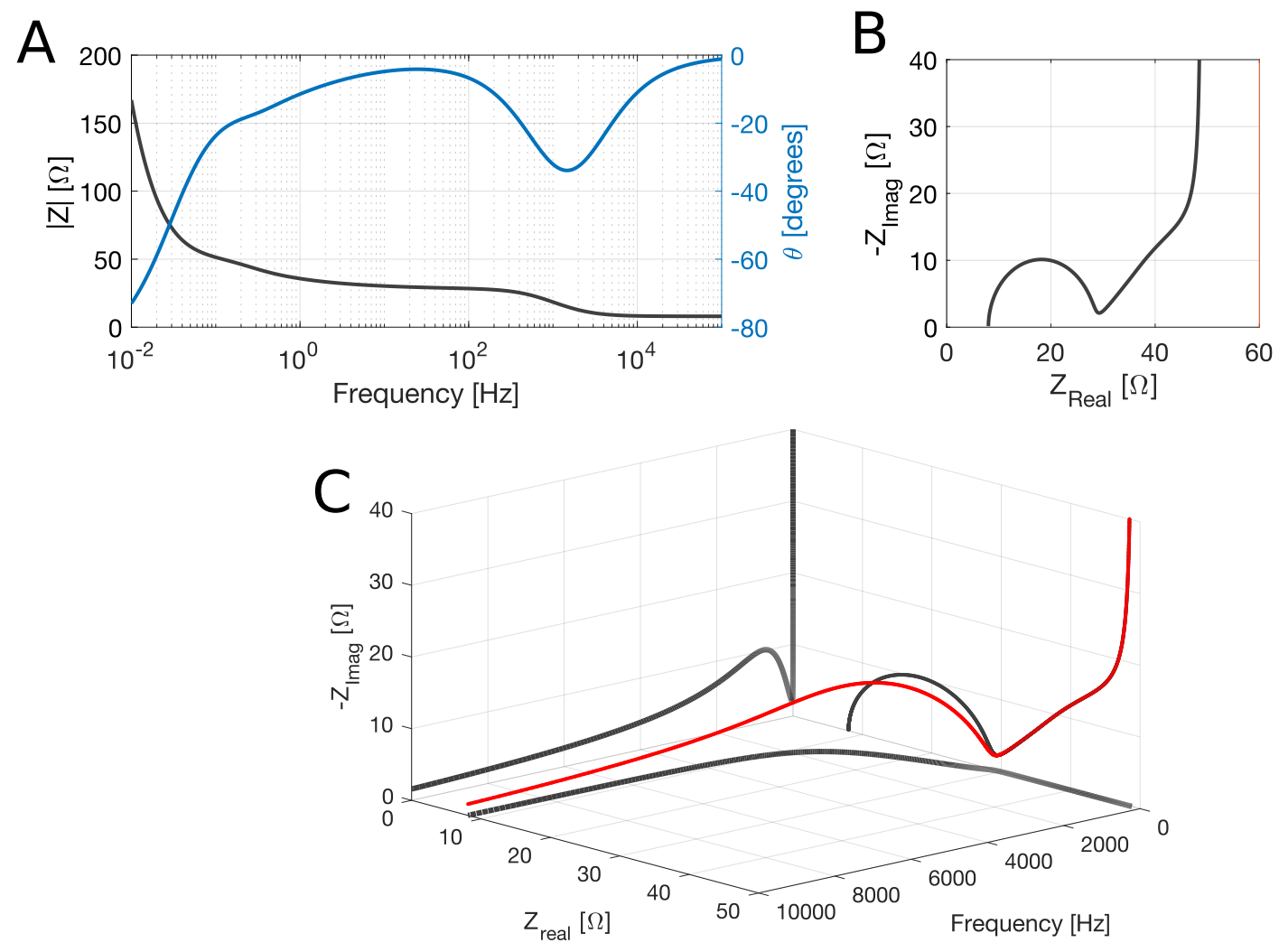

Figure 2.7: Data representation of EIS curves obtained through the simulation of Figure 2.6. A) The conventional representation of complex impedance data through magnitude and phase Bode plots. B) A Nyquist plot with a clear high frequency semicircle from the CPE followed by the $45^{\circ}$ Warburg diffusion dominance as the frequency decreases further. C) A 3D representation of the impedance data showing all aspects of the impedance data.

\subsubsection{Equivalent Circuit Modeling of Electrochemical Systems}

Packaging the mechanisms present in an electrochemical capacitor as discrete circuit components allows for quantitative interpretation of EIS results [38], [39]. Unfortunately ECM has two major difficulties: in simple electrochemical systems the EIS data can be non-unique thus multiple models could closely fit the same data [39]; secondly, detailed models can have $>7$ parameters to fit to complex EIS data making low error, high confidence parameter fitting difficult due to a large, high complexity solution space $[42]-[44]$. These challenges demonstrate the importance of correctly identifying the potential dominant processes through electrochemical theory then selecting or adapting known models to use. For electrolytic/electrode systems there exists 4 common component types. Table 2.1 shows their impedance equation. 
Table 2.1: Summary of common equivalent circuit model components and their corresponding impedances equations.

\begin{tabular}{ccc} 
Component & Symbol & Impedance \\
\hline \hline Resistor & $\mathrm{R}$ & $R$ \\
\hline Capacitor & $\mathrm{C}$ & $(j \omega C)^{-1}$ \\
\hline Inductor & $\mathrm{L}$ & $j \omega L$ \\
\hline Constant Phase Element & $\mathrm{CPE}$ & $1 /\left(Q_{\mathrm{CPE}}(j \omega)^{\alpha}\right)$ \\
\hline Warburg - Infinite Diffusion & $\mathrm{W}$ & $\left(Q_{W} \sqrt{j \omega}\right)^{-1} \tanh [\delta / \sqrt{D} \sqrt{j \omega}]$ \\
\hline Warburg - Finite Diffusion & $\mathrm{W}$ & $\left(Q_{W} \sqrt{j \omega}\right)^{-1} \operatorname{coth}[\delta / \sqrt{D} \sqrt{j \omega}]$ \\
\hline
\end{tabular}

Using these components the electrochemical phenomenon outlined in Section 2.4 can be represented by their impedance contributions. Combining the effects appropriately can allow for the recreation of the Nyquist plots and thus the fitting to experimental data.

\subsubsection{ECM Models}

By relating electrochemical mechanisms to components one can develop full models for different systems. A simple electrochemical model is the Randles Circuit seen in Figure 2.8 [39].

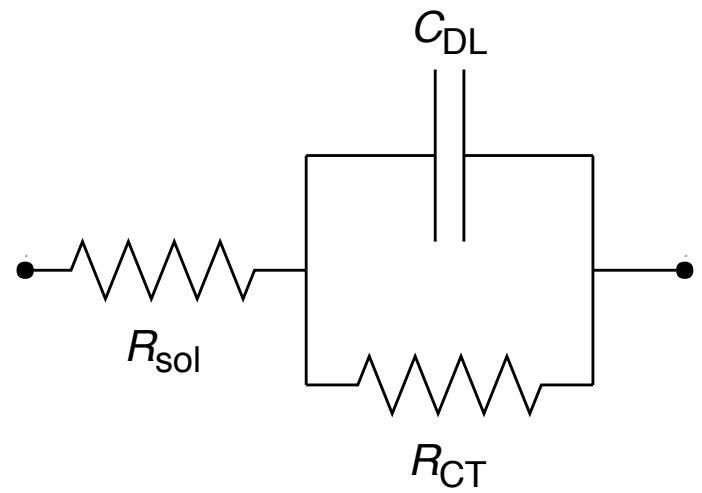

Figure 2.8: Randles Circuit

The model describes a well isolated conductive, ionic aqueous solution taking into account a series solution resistance, $R_{S o l}$, an ideal capacitor for the EDL, $C_{D L}$, in parallel with a charge-transfer resistance, $R_{C T}$. Parallel $\mathrm{RC}$ components gives rise to a semi-circular shapes seen in the Nyquist 
plot.

The Randles circuit provides a valuable base from which more advanced models usually begin. In order to describe an electrolytic capacitor, we can begin with an ideal EDLC with a non-planar electrode. In Figure 2.9 we see the Helmholtz layers and diffuse layer capacitance in series while we place the electrolytic impedance in parallel. The Warburg element is to account for the semi-infinite, or finite diffusion impedance due to a bulk separation and ion diffusion path to the electrode.

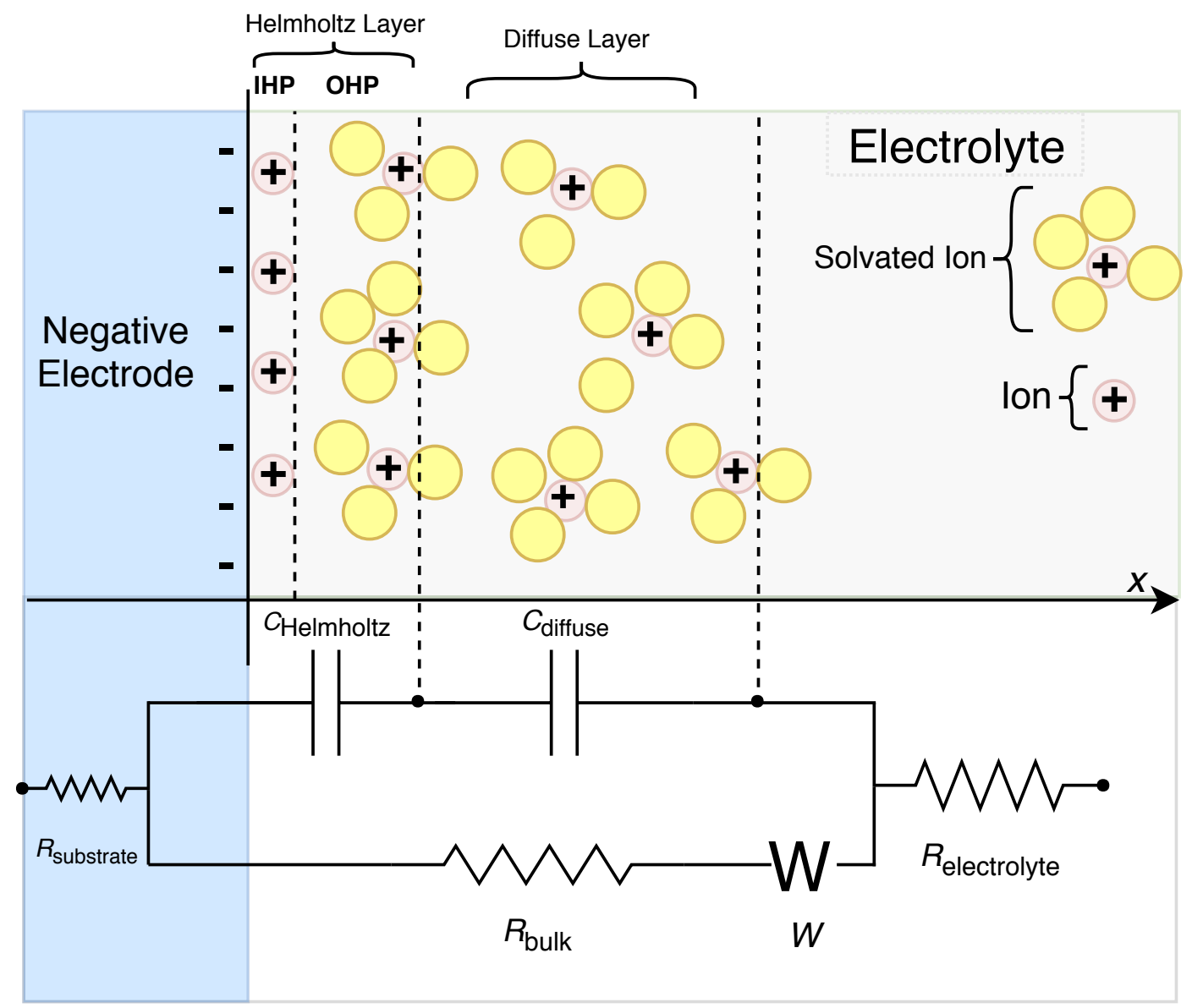

Figure 2.9: Approximately tying the equivalent circuit model to the physical regions of the surface of the EDL.

EDLCs use high surface area electrodes to maximize their energy storage, but these porous electrodes add mass-transfer latency throughout the system as the ion path is now a maze. To investigate a porous electrode through EIS the kinetics will present themselves in diffusion impedances. The Warburg element in series to the EDL and interface phenomenon can be used to describe the diffusion impedance behavior with the added latency. In addition, replacing capacitors with a constant-phase 
element (CPE), as their low frequency (LF) impedance behavior captures what has been seen in porous electrode experimental data, will improve the ECMs representation of the system [9], 45], [46]. The CPE's impedance is represented by, $1 /\left(Q_{w} j \omega\right)^{\alpha}$, where the exponent, $\alpha$, is 1 for an ideal capacitor but $\alpha$ 's deviation from 1 corresponds porous electrode effects, and ion intercalation into a material impacting the impedance [46], [47].

For EIS analysis the components must be separable by their frequency response, thus grouping all the substrate, interconnect, and the electrode resistance as the frequency independent series resistance of the device, $R_{S}$, greatly reduces the number of components. These few components accurately describes EDLCs in which the energy storage is purely through the EDL.

\subsubsection{Electrochemical Capacitor Models}

The models presented above are effective for pure EDLCs (strictly EDL capactance) but fail to account for any adsorption, intercalation, nor the presence of charge-transfer phenomenon. In recent years the EC models have evolved in the ideal form of Figure 2.10, where the $C_{H}$ is being used to describe the kinetic phenomenon and $C_{a d s}$ accounts for the inner Helmholtz layer in which ions undergo adsorption, intercalation and charge-transfer phenomenon 9 . This accounts for the pseudocapacitance exhibited by complex ECs like those with conducting polymer electrodes. [37.

The Warburg element here can be either semi-infinite or finite diffusion depending on the availability of ions and the diffusion length. The researchers, [37], extended the model to have a CPE for all capacitive components to improve the quality of the fit to their data. This is a common practice but does complicate the physical interpretation of the results in addition to the CPE creating additional parameters and adding non-linearity to the model. The CPE's interpreation itself is also debated and still becoming more established [46]. A valuable aspect of the CPE's function is the increased ability to fit the nyquist plot, enabling data fitting in complex systems. The further understanding

\footnotetext{
${ }^{9}$ The charge-transfer phenomenon are voltage mediated, thus at the low voltage EIS testing these effects are often not present. During CV and higher voltage testing (Or during operation) the adsorption layer ions can participate in redox reactions.
} 
has to be conducted with assumptions about the mechanisms present in the system, such as possible non-reflective boundary conditions of organic materials. The change in conductivity, morphology or other non-stable aspect of the device can be captured by a CPE [38], [39].

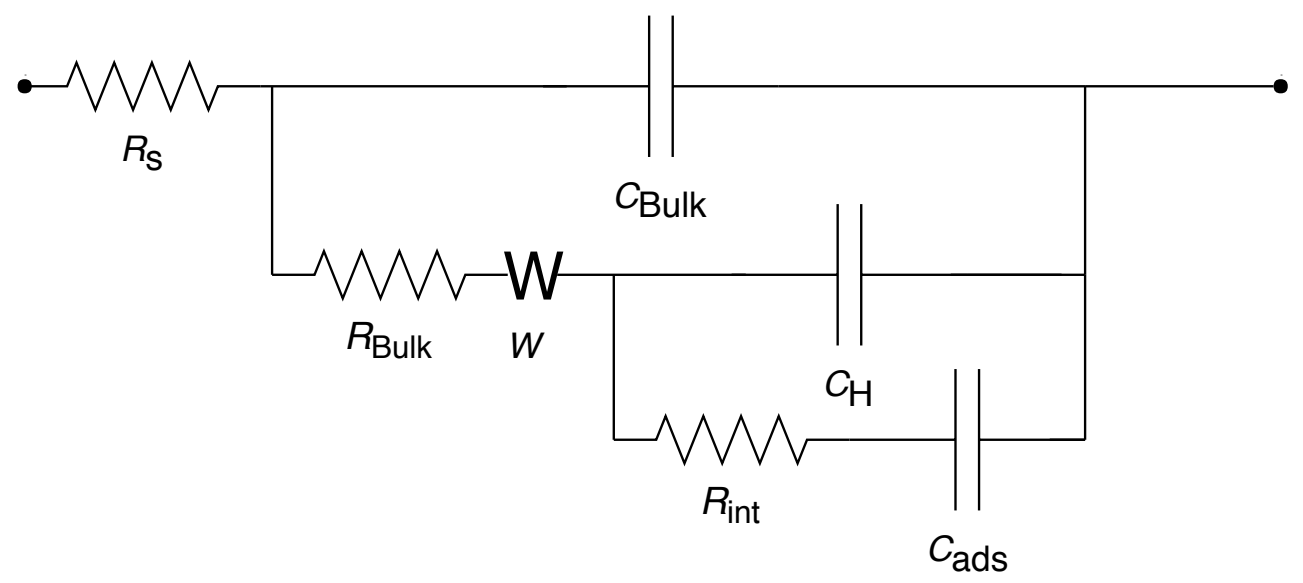

Figure 2.10: Ideal ECM to deal with the intricate interactions of the impedance contributions of the double layer and adsorption layers.

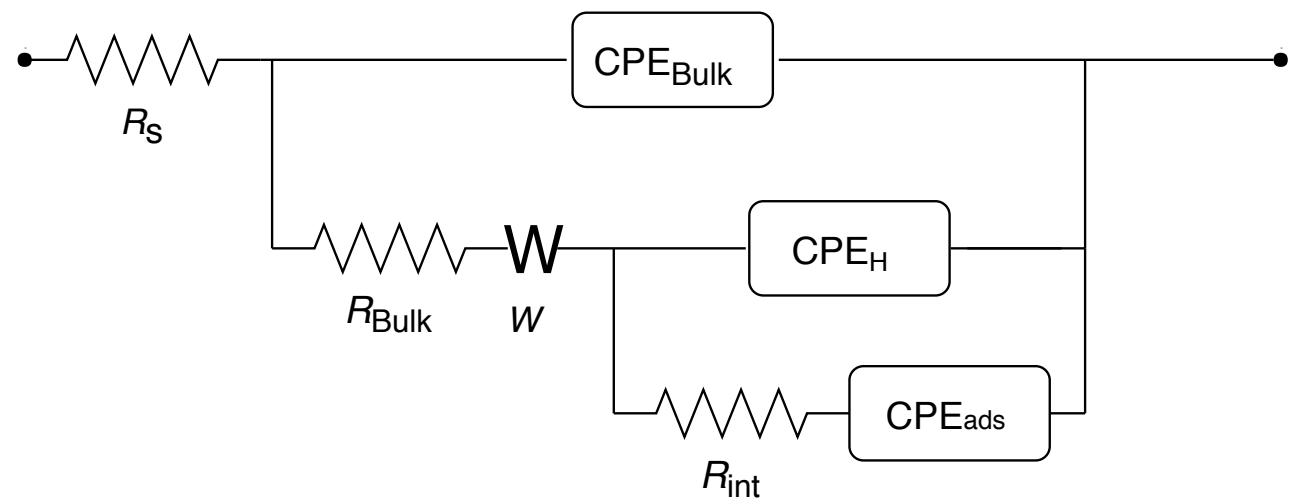

Figure 2.11: State-of-the-art model presented for highly porous EDLC electrodes shown first by 37.

\subsubsection{EIS data fitting}

With a model selected and experimental data found, the final stage to EIS is extracting the parameters. Depending on the complexity of the system some parameters can be extracted from the Bode and Nyquist plots by looking at specific shapes and intersections present, as highlighted in Figures 2.8 and 2.7. In order to more accurately tune these parameters and extract more nested parameters, the model's impedance equations can be fit to the data using a host of different algorithms. 
EIS data fitting often uses high variable, complex, nonlinear equations, all of which increase the difficulty of creating a unique, high confidence fit [42], [43]. Data fitting is a vast body of work. To approach this problem there are a host of available EIS data fitting softwares which often using either the Levenberg-Marquardt algorithm (LMA) or the Trust-Region Reflective algorithm (TRR). Unfortunately, these algorithms and software are highly sensitive to initial conditions and bounds, requiring specific extensive knowledge about the electrode or components to vastly restrict the bounds and solutions in order for the algorithms to find the correct minima. 


\section{Chapter 3}

\section{Electrospinning PEDOT:PSS Electrodes}

\subsection{Introduction}

In order to construct the electrolytic capacitors, the first step was to establish a working electrospinning process to deposit ultrahigh surface area PEDOT:PSS electrodes. The goal was to determine the required solution composition to electrospin PEDOT:PSS with PVA or PEO as the carrier polymer. Both of these polymers are inexpensive and available as high molecular weight linear chains which have demonstrated an ability to be easily electrospun. This was approached with the principle of finding the simplest effective solution. The carrier polymers (PEO and PVA) selected for easy removal, ability to be compliment the electrolyte system, and their ability to be electrospun without requiring a solvent. This chapter will recount the establishment of the electrospinning process chronologically. Beginning with PEO and PVA, a range of electrospinning parameters were determined for viable electrospinning with the constructed apparatus. Next, Baytron-P, a PEDOT:PSS ink was incorporated into the electrospinning solution in different proportions to PVA and PEO until a conductive fibre was found. The final step was to optimize and improve on leading PEDOT:PSS ES by establishing a process and solutions which contains the highest PEDOT:PSS to carrier polymer ratio with the incorporation of DMSO as a CEA. 


\subsection{Experimental Setup and Method}

\subsubsection{Electrospinning Apparatus}

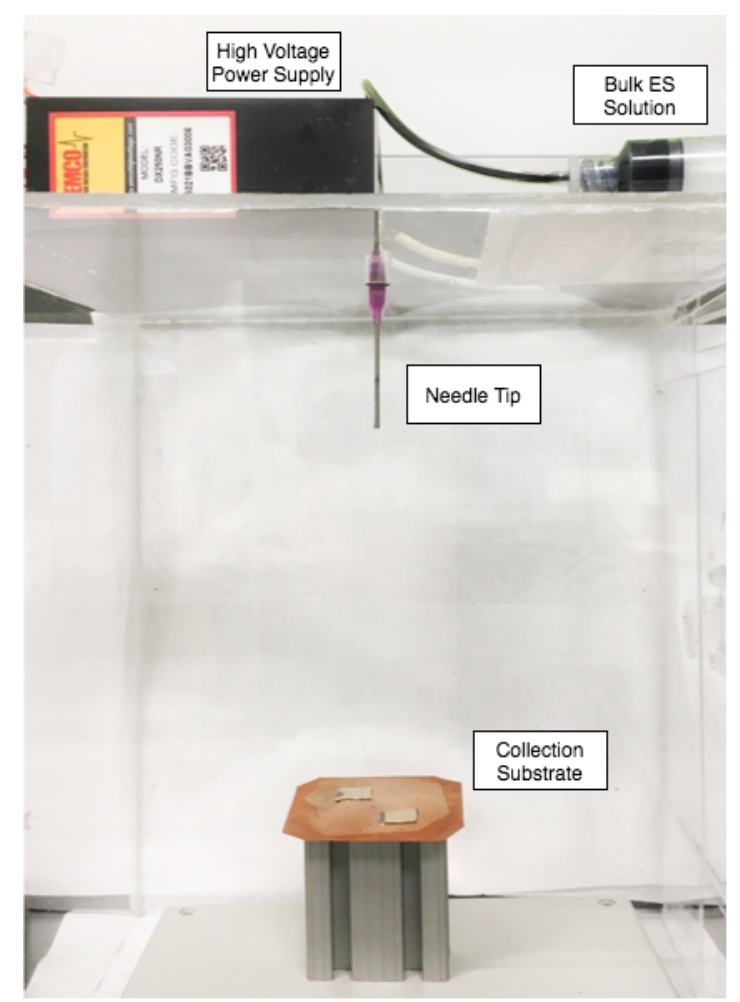

Figure 3.1: Electrospinning apparatus with the EMCO DX250 high voltage supply and a gravity fed $30 \mathrm{~mL}$ Luer Lock syringe connected to a blunt-tip needle by chemically resistant tubing. Here a grounded copper plate with small $\mathrm{Au}$ on Si samples are acting as the collector.

Using the principles of electrospinning outlined in Section 2.3, the apparatus seen in Figure 3.1 was used. The housing is a $28 \mathrm{~cm} \times 28 \mathrm{~cm} \times 40 \mathrm{~cm}$ plexiglass enclosure, using a gravimetrically fed disposable $30 \mathrm{~mL}$ Luer Lock syringe into Tygon PVC chemical resistant 1/16" tubing terminating into a stainless steel quick-turn barbed plug fixed to the top of the plexiglass container. Here a stainless steel blunt-tip $24 \mathrm{G}$ needle can be attached to the plug with all components purchased through McMaster-Carr. The needle and plug were electrified using an EMCO DX250 high voltage DC Converter for $10 \mathrm{kV}-25 \mathrm{kV}$, and the EMCO Q101 and Q101N for the positive and negative 0 $10 \mathrm{kV}$ supplies; Collection substrates were grounded on a small cooper back plate mounted to 80/20 extruded aluminum tubing. The collection electrodes used a silicon wafer with shadow masked 
patterned gold, more details regarding the silicon wafers can be found in Chapter 4 .

\subsubsection{Electrospinning Method}

Electrospinning is a balance of a host of different parameters that are not easy to measure during deposition. In order to determine the ideal spinning parameters for each solution the rigid experimental procedure shown in the flowchart seen in Figure 3.2 was followed. The method aims to be versatile and explore the simply adjusted variables first to avoid additives by exhaustive search through the dominant parameters.

When a solution found is to be compatible with electrospinning, a few qualitative factors of the fibres were recorded with each deposition: beading, stability of process, and uniformity. These factors are commonly associated with the potential of the solution to be ES [33].

\section{Conductivity Testing}

To quickly approximate the resistance of the PEDOT:PSS fibres, the successful solutions were deposited onto a EC test substrate, described in detail in Chapter 4, and the resistance was measured between both the EC device contacts for a fixed distance. The known dimensions of the contacts were used to very roughly approximate a sheet resistance for comparison against other devices within the work in $\Omega /$ sq using Equation 3.2 .2 .

$$
R_{\text {sheet }}=R_{\text {measured }} \frac{W}{L}
$$

This simplistic approach to finding the resistance was used to create a standard to compare against other fibres within this body of work. The resistance of the electrodes will change due to ion intercalation during operation, and the resistance of the ES electrodes will be investigated more thoroughly through EIS in Chapters 5 and 6. 


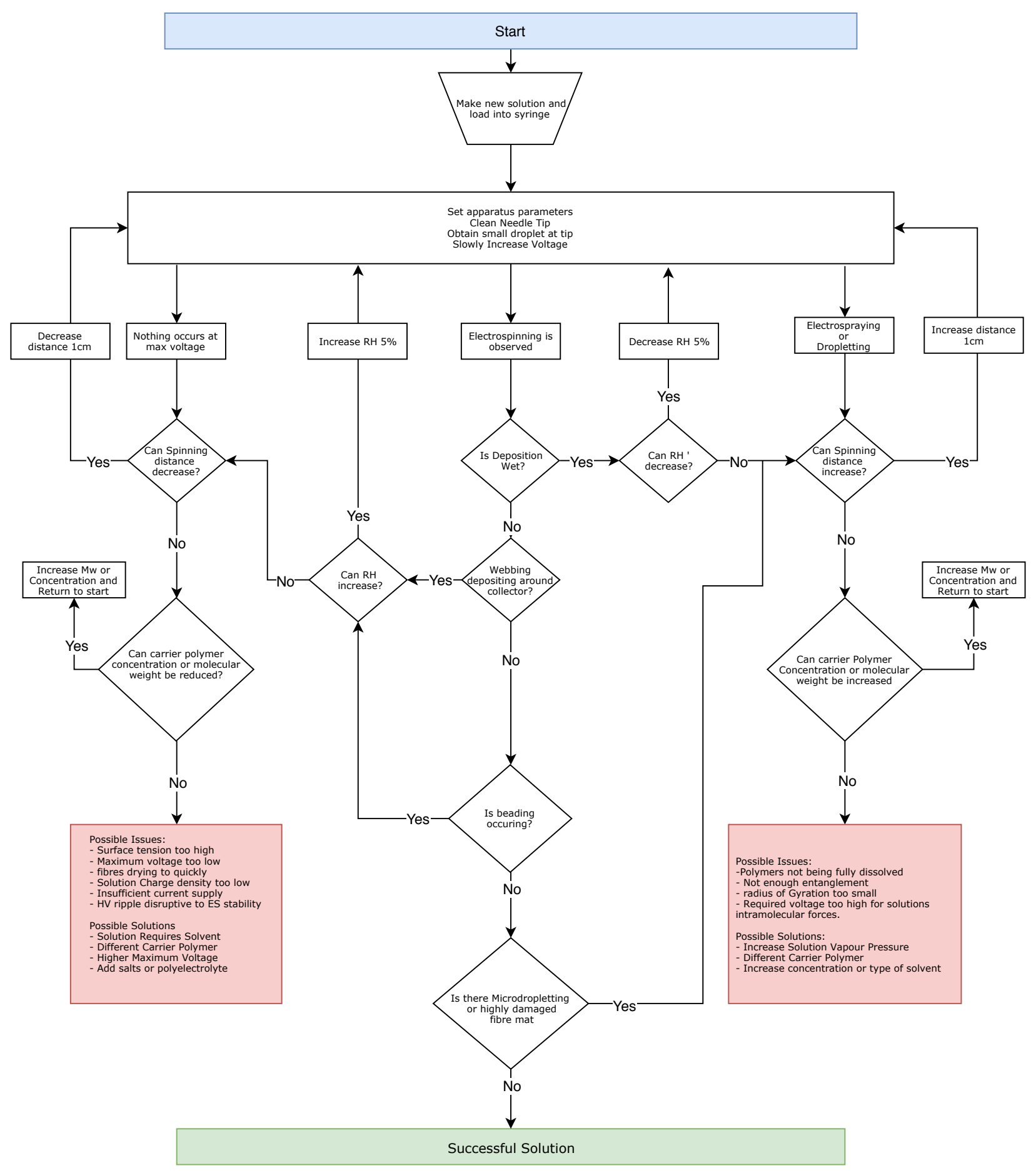

Figure 3.2: This deicison making process was used to troubleshoot ES solutions until electrospinning was consistently observed and was of the quality needed for this work. The flow chart omits the steps required to make the solution. 


\subsection{Initial Process Tuning}

Aqueous test solutions of PEO and PVA were used to verify the range of parameters for the electrospinning process. PEO and PVA were selected as their high molecular weight, non-toxicity, and molecular simplicity have shown wide use in the ES field. In addition, PEO and PVA have demonstrated compatibility with the desired electrolyte system and their solubility in water allows for easy removal from the PEDOT:PSS fibres.

To begin, some accepted literature concentrations were identified and were used to gauge a starting point for different molecular weights [48]. The solutions were prepared adding $M_{v}=5,000,000 \mathrm{PEO}$ powder and $M_{v}=40,000-70,000$ hydrated PVA powder sourced from Sigma Aldric added to DI water. Solutions with PEO weight concentrations of 1.25\%, 1.5\%, 1.75\%, and $2 \%$ and PVA weight concentrations of $4 \%, 5 \%, 6 \%$, and $7 \%$ by mass were made by weighing the respective powders and adding to a $20 \mathrm{~mL}$ glass bottle containing $5 \mathrm{~mL}$ of DI water. A magnetic stirring rod was added and the solution was mixed for a minimum of $48 \mathrm{~h}$ allowing the solution to be uniform and clear. The results of the tuning process are summarized below in Table 3.1

Table 3.1: Results of the tuning sweep of $M_{v}=5,000,000$ PEO/water, and $M_{v}=40,000-70,00087 \%-89 \%$ hydrolyzed PVA/water solutions to determine electrospinning apparatus parameters and carry polymer behaviors.

\begin{tabular}{ccccccc} 
Polymer & Added $(\%)$ & $\begin{array}{c}\text { ES } \\
\text { Observed }\end{array}$ & $\begin{array}{c}\text { Voltage } \\
\text { Range }(\mathrm{kV})\end{array}$ & $\begin{array}{c}\text { Distance } \\
(\mathrm{cm})\end{array}$ & $\begin{array}{c}\text { Humidity } \\
(\mathrm{RH})\end{array}$ & Details \\
\hline \hline PEO & 1.25 & No & N/A & N/A & N/A & Electrospraying \\
\hline PEO & 1.5 & Yes & $15-25$ & $20-50$ & $10-30$ & Wet, uniform \\
\hline PEO & 1.75 & Yes & $18-25$ & $20-50$ & $5-20$ & Wet, uniform \\
\hline PEO & 2 & Yes & $18-25$ & $20-50$ & $5-20$ & Wet, uniform \\
\hline PVA & 4 & Minor & N $/ \mathrm{A}$ & N/A & N/A & Electrospraying \\
\hline PVA & 5 & Yes & $8-15$ & $12-25$ & $10-30$ & Webbing, uniform \\
\hline PVA & 6 & Yes & $8-15$ & $12-25$ & $10-30$ & Webbinor dropletting \\
\hline PVA & 7 & No & N $/ \mathrm{A}$ & N $/ \mathrm{A}$ & N/A & Electrospraying \\
\hline
\end{tabular}

Using Equation 2.3 and applying the Mark-Houwink Equation seen in 2.2 one finds the approximate 
minimum entanglement concentration for PEO and PVA in water.

Table 3.2: Approximate values for entanglement concentrations from Mark-Houwink equation of PEO and PVA in water. Parameters sourced from Reference 49]

\begin{tabular}{cccc} 
Polymer & $\kappa(\mathrm{L} / \mathrm{g})$ & $\mathrm{a}$ & $\mathrm{c}_{\text {entanglement }}(\%)$ \\
\hline \hline PEO & 0.000125 & 0.78 & 0.0476 \\
\hline PVA & 0.00666 & 0.640 & 0.12 \\
\hline
\end{tabular}

Using $c_{\text {entanglement }}$ seen in Table 3.2 we find the successful ratio of the electrospinning concentration, $C_{E S}$, to the entanglement concentrations $c_{E S} / c_{\text {entanglement }}$ for the different solutions to provide an approximate understanding of the starting concentrations needed with the successful apparatus parameters used throughout this work, summarized in Table 3.3 .

Table 3.3: Approximate values for entanglement concentrations from Mark-Houwink Equation of PEO and PVA in water.

\begin{tabular}{ccc} 
Polymer & Min $c_{E S} / c_{\text {entanglement }}$ & Max $c_{E S} / c_{\text {entanglement }}$ \\
\hline \hline PEO & 31.52 & 42.02 \\
\hline PVA & 33.33 & 50 \\
\hline
\end{tabular}

Here we see that this very crude approximation does yield similar ranges (despite them being non-exhaustive). These ratios provide a range of ratios to be used to begin testing with different materials or with perturbations to the solution. These ratios also provide insight to the magnitude of intermolecular forces between polymers (entanglement) needed to sustain electrospinning with the given apparatus parameters.

\subsection{PEDOT:PSS Conductive Nanofibres}

\subsubsection{Solution Tuning}

To obtain conductive PEDOT:PSS fibres, the successful carrier polymer proportions found in Section 3.3 were used with Baytron P substituting the DI water. Baytron P is a PEDOT:PSS solution with a 1:2.5 PEDOT:PSS ratio and 1.3\% solids content by weight used in OLED and OFET applications. The PEO in Baytron P demonstrated a large amount of clumping and beading on the fibres as well 
as issues regarding fully dissolving within the ink1. As for the PVA, it did demonstrate an ability to disolve fully but an unstable electrospinning with frequent electrospraying was observed. This led to the combination of the two polymers. Following the findings from Table 3.3 , a PVA solution of $4 \%$, found to be on the edge of the electrospinning threshold, alone, had $0.15 \%, 0.25 \%$, and $0.5 \%$ of PEO added for a new batch of solutions. Following the method outlined in Section 3.2 viable PEDOT:PSS fibres were deposited with the best results being found being a solution of $4 \%$ PVA and $0.5 \%$ PEO dissolved in Baytron P. Finding that required higher baseline $c_{E S} / c_{\text {entanglement }}$ ratio of 43.84 was needed for the Baytron P substitution resulting in a solution with an approximate solid content of $5.74 \%$. These fibres did experience some damage from electrospraying but was minimal in most cases.

Table 3.4: Electrospinning apparatus parameters for PEDOT:PSS/PVA/PEO solution for conductive nanofibres.

\begin{tabular}{ccc} 
Parameter & Lower Bound & Upper Bound \\
\hline \hline Needle Voltage & $14 \mathrm{kV}$ & $16 \mathrm{kV}$ \\
\hline Collector Voltage & GND & GND \\
\hline Spinning Distance & $15 \mathrm{~cm}$ & $25 \mathrm{~cm}$ \\
\hline Humidity & $15 \% \mathrm{RH}$ & $20 \% \mathrm{RH}$ \\
\hline
\end{tabular}

The PEDOT:PSS/PVA/PEO fibres were found to have a resistance of $3.8 \mathrm{M} \Omega / \mathrm{sq}$ straight from deposition and had an average fibre diameter of $143 \mathrm{~nm}$ as seen in Figure 3.3. The SEM investivation of the fibres was conducted with a Tescan Vega-II XMU scanning electron microscope (SEM) with an accelerating voltage of $3 \mathrm{kV}-20 \mathrm{kV}$. The current density and voltage were kept at a minimum to reduce sample heating and minimize the microscopy's influence on the material. In order to improve the conductivity, post treatment techniques with CEA were investigated.

\footnotetext{
${ }^{1} \mathrm{PEO} /$ Baytron $\mathrm{P}$ was further investigated at this time with DMF, and Ethlyene as common ES solvents but was unsuccessful at producing an electrospinning viable solution with the apparatus parameters available.
} 


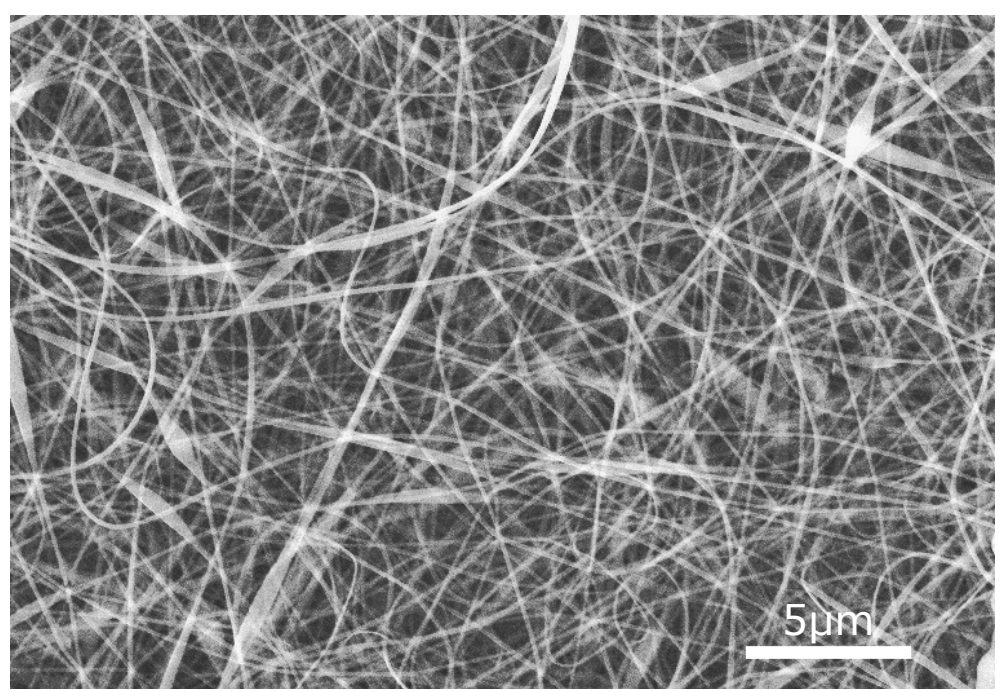

Figure 3.3: SEM of deposited ES PEDOT:PSS/PVA/PEO fibres collected onto Au on Silicon wafer.

\subsubsection{Post-Processing}

As a leading method to treating PEDOT:PSS, DMSO was used to treat the deposited mat followed by curing at $120^{\circ} \mathrm{C}$ for 20 minutes, as suggested in literature [30], [31]. The post processing treatment demonstrated improvements in conductivity if the fiber mat survived, as often the fibres had been washed away or heavily damaged in the process as Figure 3.4 shows. In addition, the fibres had a reduced average thickness, showing that some some material had been removed from each fibre.
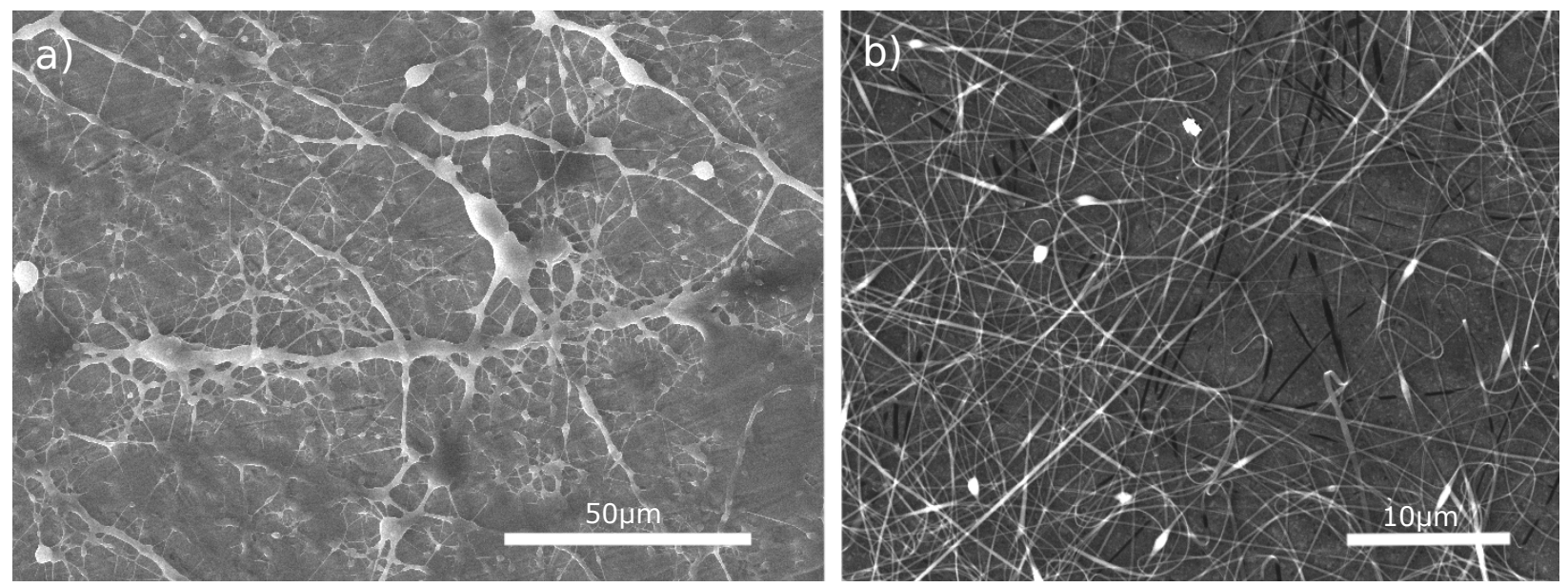

Figure 3.4: SEM image of the PEDOT:PSS/PVA/PEO fiber after different DMSO treatments. (a) Given longer DMSO exposure the fibre was observed to melt or dissolve the fibres forming a rough film on the surface of the electrode. (b) the wafer was dipped into a DMSO solution, stripping the fibres off of the mat.

The results of the PEDOT:PSS/PVA/PEO fibers incorporated in an electrolytic capacitor structure 
and were presented at MRS 2018 [50]. The EDLC performance results of these fibres are found in Chapter 5. It is found that a high contact resistance and a dispersed low density mat could be limiting to the functionality of the device. To improve the electrodes, higher conductivity ES fibres are needed.

\subsection{Optimization: PEDOT:PSS/PEO}

\subsubsection{Solution Tuning}

Two major improvements were sought out to improve on the limitations of PEDOT:PSS/PVA/PEO fibres. First, to increase the PEDOT:PSS content of the fibres to allow for more active material within the fibre and electrode. Second, to find a viable post processing treatment to increase the fibre mat density while increasing conductivity through both increased fibre to fibre connection and conformation of the PEDOT within the fibre. Parallel work by 25] demonstrated that simply PEDOT:PSS/PEO can be successfully electrospun. To build on their results by achieving a higher PEDOT:PSS to carrier ratio the following adjustments to the process were made:

- UHMW PEO $\left(M_{w}=5,000,000\right)$ : High molecular weight to require the smallest amount of carry polymer to achieve entanglement provided a high PEDOT:PSS to carrier polymer fibre composition.

- 4 - 5 day mixing times: Longer mixing times to ensure proper mixing of PEO and PEDOT:PSS.

- Mixing temperature increased to $35-40{ }^{\circ} \mathrm{C}$ : A slight increase in temperature to encourage mixing of the polymers.

- Positive 5 - $10 \mathrm{kV}$ on needle: Lower needle voltage to discourage dropletting and electrospraying.

- Negative 0 - $5 \mathrm{kV}$ on Collector: Small applied voltage to compensate for the reduced needle voltage and to electrostatically encourage deposition onto the collection substrate over the neutral, floating apparatus walls. 
- RH kept above $25 \%$ : Higher humidity discouraging beading for smoother depositions for increased conductivity.

- Spinning distance decreased to 10 - $15 \mathrm{~cm}$ : Shorter spinning distance compensating for the reduced needle voltage.

These adjustments were made to the apparatus parameters and method to build on the previous results and to encourage uniform, confined depositions onto the collection substrate discouraging a wide spread of fibers while obtaining conductive ES fibres.

The solutions were prepared adding $M_{v}=5,000,000$ UHMW PEO powder in $1.2 \%, 1.45 \%, 1.6 \%$ concentrations with DMSO concentrations of $5 \%$ and $10 \%$. The results of the tuning process are summarized below in Table 3.5 with the best results from being 1.4\% PEO with 5\% DMSO, with SEM images shown in Figure 3.5 .

Table 3.5: Summary of qualitative electrospinning results of Baytron $\mathrm{P} / \mathrm{PEO} / \mathrm{DMSO}$ in $1.2,1.4$, or $1.6 \%$ by wt PEO and $5 \%$ or $10 \%$ DMSO by wt solutions. Baytron P contains $1.3 \%$ by wt PEDOT:PSS solids. The $1.4 \%$ wt PEO with $5 \%$ DMSO demonstrated conduction with the lowest carrier polymer concentration investigated.

\begin{tabular}{ccccc}
$\begin{array}{c}\text { PEO } \\
(\% \mathrm{wt})\end{array}$ & $\begin{array}{c}\text { DMSO } \\
(\% \mathrm{wt})\end{array}$ & $\begin{array}{c}\text { Electrospinning } \\
\text { Observed }\end{array}$ & $\begin{array}{c}\text { Conduction } \\
\text { Observed }\end{array}$ & Qualitative Process Observations \\
\hline \hline 1.25 & 5 & Yes & No & Heavily damaged, dropletting, unstable \\
\hline 1.25 & 10 & No & No & Unstable, only electrospraying \\
\hline 1.45 & 5 & Yes & Yes & Highly uniform, stable, confined \\
\hline 1.45 & 10 & Yes & No & Inconsistent, electrospraying \\
\hline 1.65 & 5 & Yes & Yes & Uniform process with some dropletting \\
\hline 1.65 & 10 & Yes & Yes & Heavily damaged, inconsistent, dropletting \\
\hline
\end{tabular}

In order to create better than literature ES PEDOT:PSS fibers, post-treatment had to be investigated. Literature has demonstrated the ability for the fibres to be post-treated with DMSO without destruction but the details of their steps are not outlined. Initially a straight DMSO treatment was pursued with the PEDOT:PSS/PEO fibre. This resulted in the damaging or even complete dissolution of the nanofibre mat. The mat demonstrated improvement in conductivity but the closure of the pores makes this a non-ideal processing technique. 

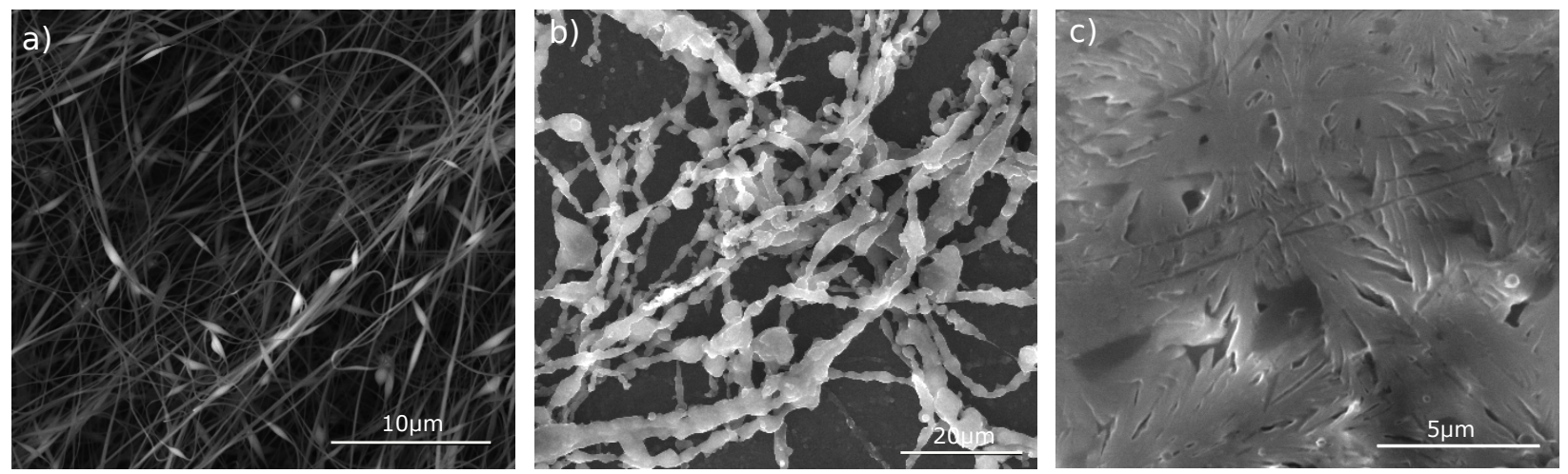

Figure 3.5: SEM images of ES PEDOT:PSS/PEO fibres at different states in processing: (a) the fibres directly after electrospinning collected onto a Au electrode on silicon; (b) is the fibres of Image a) after being post processed thermally, and with DMSO; (c) presents the fibres after $48 \mathrm{~h}$ of exposure to lithium perchlorate/proplyene carbonate electrolyte while undergoing lifecycle testing. During the investigation of samples after electrolyte exposure, high resolution imaging did result in melting of regions of the nanofibre mat. This resulted in the inability to image the mat in higher detail than what is seen in (c).

Acetone, a weaker solvent, was selected to pretreat the ES fibre mat, as acetone presents both polar and non-polar regions and does not readily dissolve PEDOT:PSS [26], [51]. This was followed by drying for 10 minutes at $60^{\circ} \mathrm{C}$ then ES fibre mats were submerged in DMSO and cured for 20 minutes at $110^{\circ} \mathrm{C}$. The ES fiber mat post deposition was found to be thin fibres with small amounts of beading, seen in Figure $3.5 \mathrm{a}$. The post treatment of the fibre did show significant clumping of the fibers causing an extreme increase in the fibre diameter shown in Figure 3.5b. This result was unexpected as the fibre mat when tested as an EDLC (Seen in Chapter 6) demonstrated performance improvements. To investigate further, electrodes after testing were imaged, seen in figures 3.5;, where pores and gaps in the surface of the thick clumped fibres have begun to appear. The more film-like electrode is encouraging as this allows for an increase in volumetric energy density. The appearance of pores in the fibre mat is most likely the PEO being removed by the non-polar organic solvent, proplyene carbonate (PC), in the electrolyte, as PEO can be dissolved by PC but PEDOT:PSS should not degrade as quickly [51]. The removal of PEO from the fibres allows for the surface area to increase and improves the electrolyte ion's access to the PEDOT:PSS reducing the thickness of the Helmholtz layer, increasing capacitance. In addition, the resistance of the fibres was significantly reduced during washing steps. The final measured resistances were found to be in the range of $1.3 \mathrm{k} \Omega / \mathrm{sq}-150 \Omega / \mathrm{sq}$. 


\subsection{Summary of Process}

The ES deposition for all steps was found to be a sparse mat which macroscopically distributed in a circle with the highest concentration of deposition in the center decaying radially. This distribution was exaggerated by longer deposition times.

The final ES solution found through the above mentioned ES process was Baytron $\mathrm{P} / \mathrm{PEO} / \mathrm{DSMO}$ in $93.55 / 1.45 / 5 \%$ by weight with a solution solids content of $2.67 \%$, resulting in an as deposited fibre with $46.5 \%$ PEDOT:PSS content before post-treatment. The average diameter of the fibre distribution and deposition is summarized in Table 3.6 .

Table 3.6: Summary of the diameter and distribution of the ES fibres at different stages of development.

\begin{tabular}{cccc} 
Solution & Type & Mean $[\mathrm{nm}]$ & $\sigma^{2}[\mathrm{~nm}]$ \\
\hline \hline \multirow{2}{*}{ PEDOT:PSS/PVA/PEO } & Untreated & 143 & 7.3 \\
\cline { 2 - 4 } & Post Treatment & 130 & 5.7 \\
\hline \multirow{3}{*}{ PEDOT:PSS/PEO } & Untreated & 171 & 64 \\
\cline { 2 - 4 } & Post Treatment & 2390 & 1370 \\
\cline { 2 - 4 } & Post Use & 376 & 170 \\
\cline { 2 - 4 } & Post Use Pores & 212 & 241 \\
\hline
\end{tabular}

The summary of apparatus parameters can be found in Table 3.7. This solution and parameters were used for all devices in Chapter 6 .

Table 3.7: Electrospinning apparatus parameters for PEDOT:PSS/PEO solution for conductive nanofibres.

\begin{tabular}{ccc} 
Parameter & Lower Bound & Upper Bound \\
\hline \hline Needle Voltage & $5 \mathrm{kV}$ & $7 \mathrm{kV}$ \\
\hline Collector Voltage & $-2 \mathrm{kV}$ & $-4 \mathrm{kV}$ \\
\hline Spinning Distance & $12 \mathrm{~cm}$ & $14 \mathrm{~cm}$ \\
\hline Humidity & $25 \% \mathrm{RH}$ & $35 \% \mathrm{RH}$ \\
\hline
\end{tabular}

The post treatment of the fibres demonstrated up to 5 orders of magnitude increase on conductivity. This was due to use of DMSO with a thermal curing which decreased the thickness of the mat and 
increased the fibre interconnections in addition to the conventional CEA effects. The final resistances measured laterally on the fibre mats were found to be in the range of $1.3 \mathrm{k} \Omega / \mathrm{sq}-150 \Omega / \mathrm{sq}$. The post-processing method is summarized in Table 3.8 .

Table 3.8: Summary of the final ES PEDOT:PSS post-processing steps using DMSO as a high dipole CEA for PEDOT:PSS.

\begin{tabular}{ccccc} 
Step $\#$ & Process & Time & Temp $\left[{ }^{\circ} \mathrm{C}\right]$ & Resistance Range $[\mathrm{k} \Omega / \mathrm{sq}]$ \\
\hline \hline Step 1 & Dry & $10 \mathrm{~min}$ & 60 & $\approx 200-400$ \\
\hline Step 2 & Acetone Bath & $2 \mathrm{~s}$ & $\mathrm{RT}$ & $\mathrm{N} / \mathrm{A}$ \\
\hline Step 3 & Dry & $10 \mathrm{~min}$ & 60 & $105-18$ \\
\hline Step 4 & DMSO Bath & $6 \mathrm{~s}$ & $\mathrm{RT}$ & $\mathrm{N} / \mathrm{A}$ \\
\hline Step 5 & Cure & $20 \mathrm{~min}$ & 110 & $1.3-0.152$ \\
\hline
\end{tabular}




\section{Chapter 4}

\section{EDLC Test Structure}

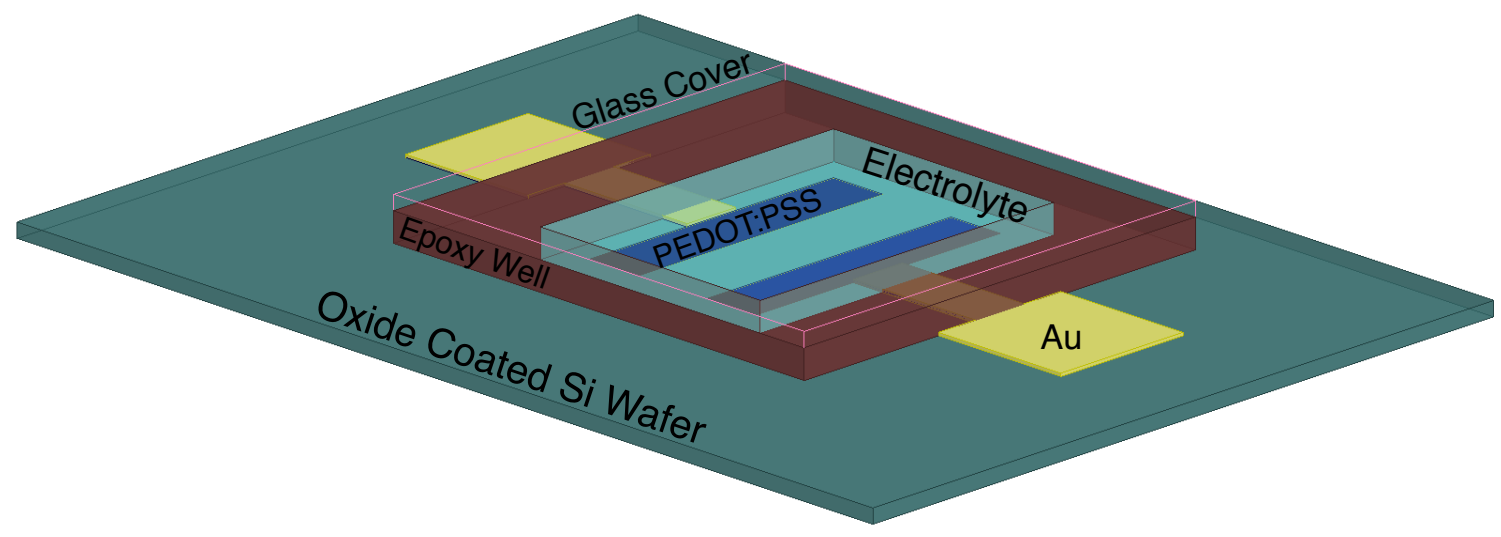

Figure 4.1: Illustration of the EDLC test structure. The structure consists of patterned gold contacts(yellow) with ES PEDOT:PSS electrodes (dark blue) sealed in a well (brown, made of UV cure epoxy) of lithium perchlorate/ proplyene carbonate electrolyte (light blue). The realized test structures were fabricated on 2-inch p-type Si wafers with $100 \mathrm{~nm}$ of thermal oxide. The Au contacts required a $10 \mathrm{~nm}$ adhesion layer of Cr.

\subsection{Components}

The testing of the ES PEDOT:PSS fibers was conducted on 2-inch Si wafers with $100 \mathrm{~nm}$ of thermal $\mathrm{SiO}_{2}$, with $75 \mathrm{~nm}$ of $\mathrm{Au}$ using a $10 \mathrm{~nm}$ adhesion layer of $\mathrm{Cr}$ patterned into the electrodes and contacts. Both $\mathrm{Au}$ and $\mathrm{Cr}$ were deposited by thermal evaporation using a Balzers BA510 evaporator. The patterning used shadow masks designed within this work using SolidWorks and were manufactured by Pela Technologies. The Au electrode and contacts were selected for their high conductivity and electrochemical stability [52]. Au is known to exhibit minor electrochemical reactions in acidic and basic environments above $0.8 \mathrm{~V}$ but these reactions are minor and should not 
be an influence given an aprotic electrolyte and polymers present during testing [52]. The EDLC test structures without ES fibres were subjected to the same battery of tests as the test devices to ensure chemical stability during operation and to establish a baseline amount of energy storage storage. These results can be found in Chapter 5 .

The final major component of the device is the electrolyte. For this work a non-aqueous 0.9 M lithium perchlorate in propylene carbonate $\left(\mathrm{LiClO}_{4} / \mathrm{CH}_{3} \mathrm{C}_{2} \mathrm{H}_{3} \mathrm{O}_{2} \mathrm{CO}\right)$ was used. An anhydrous electrolyte was used for consistency with industry which limits the voltage window around $1 \mathrm{~V} \mathrm{[7],}$ [37], [53. The presence of water in electrolyte systems gives rise to large increases in capacitance in EDLCs through the reduction in solvent ion size and increasing bulk electrolyte conductivity but can reduce lifetime due to non-reversible electrochemical reactions [7], [37], [53]. For the electrolyte, $\mathrm{LiClO}_{4}$, is a widely used and studied electrolyte/solvent system in energy storage applications as: $\mathrm{Li}^{+}$and $\mathrm{ClO}_{4}^{-}$are ions moderately sized not specifically complicating diffusion impedance and capacitance by maintaining a thin helmholtz layer, as seen through equations 2.9 and 2.4 reducing $d$, the diameter ions; high electrolyte conductivity reducing bulk resistance seen in Equation 2.10, and has chemical stability [54]. 


\subsection{Test Structure Geometry and Preparation}
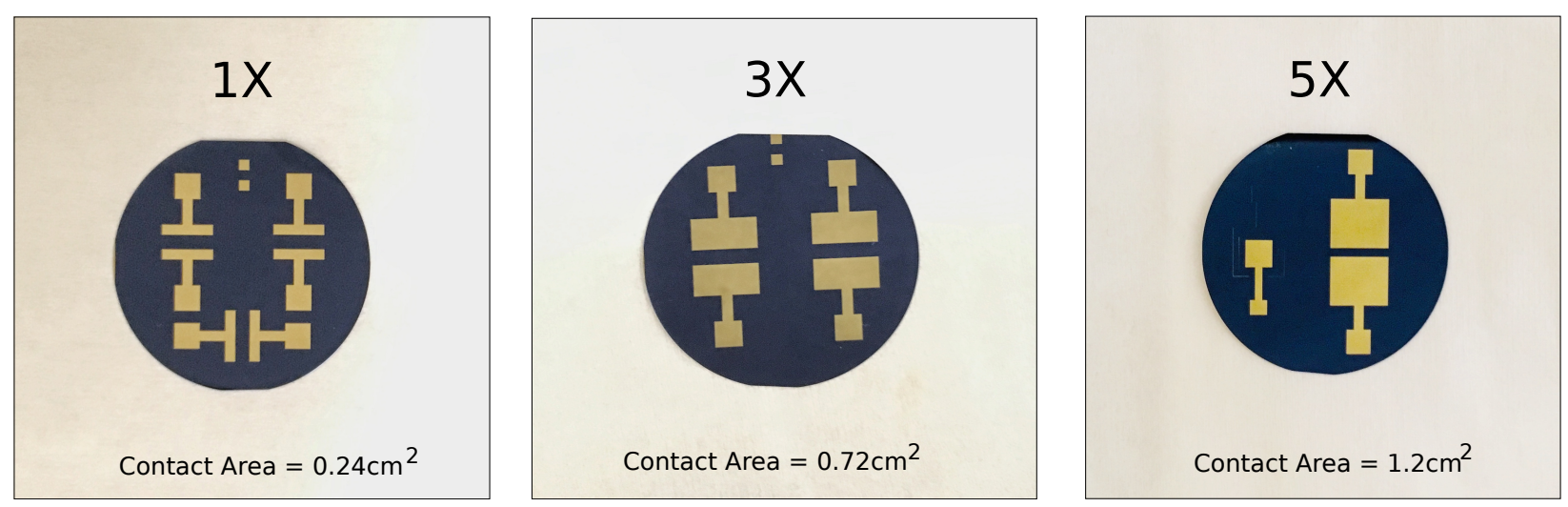

Figure 4.2: Photos of the three different wafers used in testing the ES PEDOT:PSS electrodes.

The test structure geometry consisted of two planar Au electrodes separated by a $2 \mathrm{~mm}$ gap. Three different sizes of symmetric electrode pairs of $1 \mathrm{X}, 3 \mathrm{X}$, and $5 \mathrm{X}$ surfaces area used to investigate scalibility of the result's areal capacitance, seen in Figure 4.2.
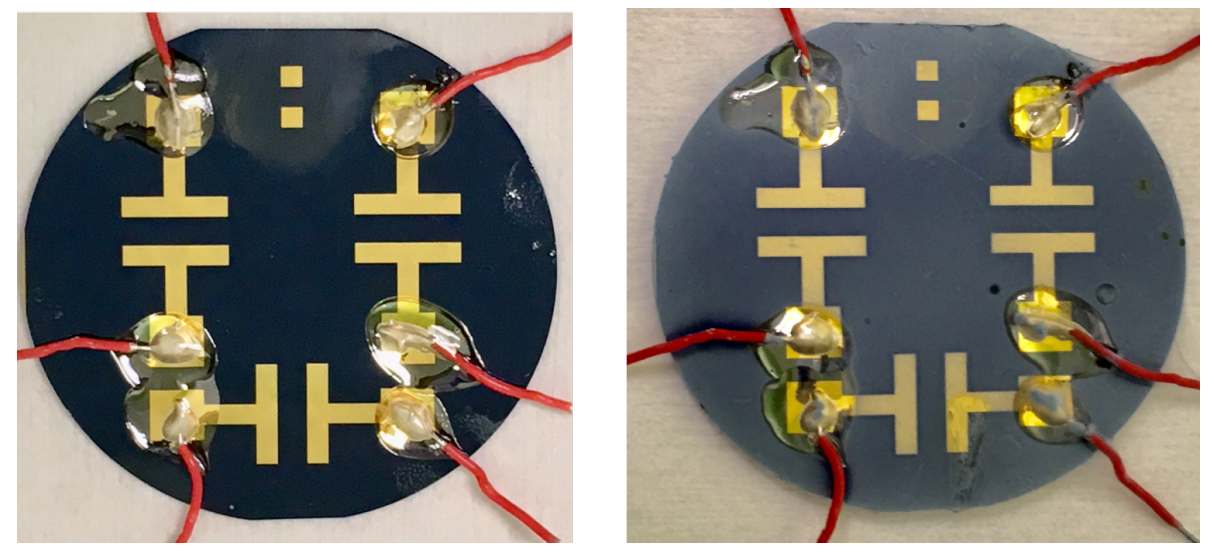

Figure 4.3: Photos of the wafers before, left, and after ES collection, right.

Figure 4.1 shows an illustration of a fully assembled test structure geometry which consists of the electrode pairs. A short wire was attached to the exposed Au contacts using thermal silver epoxy then sealed in low viscosity Norland UV epoxy. Sealing these contacts was vital as silver will electrochemically poison a $\mathrm{LiClO}_{4} / \mathrm{CH}_{3} \mathrm{C}_{2} \mathrm{H}_{3} \mathrm{O}_{2} \mathrm{CO}$ electrolyte system. ES PEDOT:PSS fibres were then collected onto the above mentioned Si wafer. Figure 4.3 shows a $1 \mathrm{X}$ test structure, left, and after 
ES collection of PEDOT:PSS PEO fibres, right, tested in Chapter 6. The wafer was post-processed following the method previously outlined in Table 3.8 .

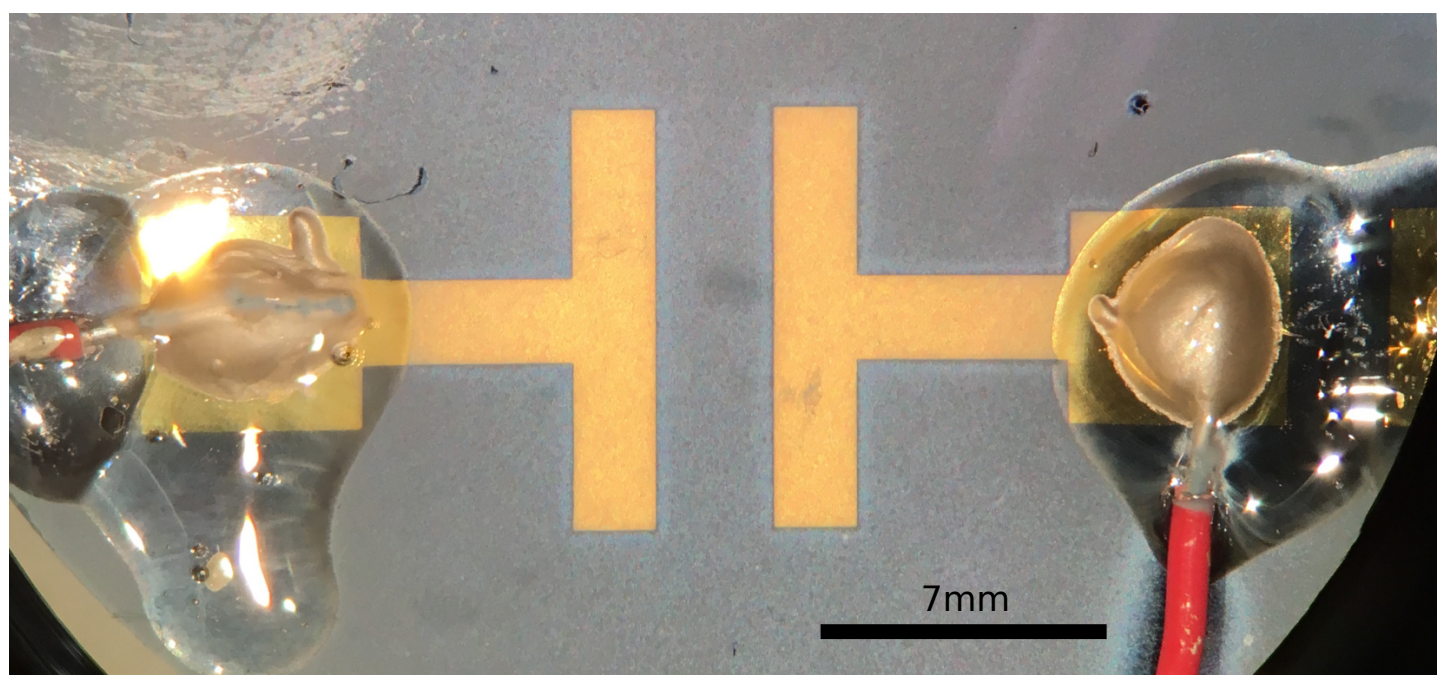

Figure 4.4: Optical microscope photo of close up of a $1 \mathrm{X}$ test structure covered with ES PEDOT:PSS. The silver epoxy is shown encased in UV cure epoxy, preventing silver poisoning of the electrochemical system.

The individual contacts are then mechanically separated using a scalpel, while also removing all excess material within the epoxy well and checked with under optical microscopy and digital multimeter to ensure separation. Figure 4.5 shows a close up of a contact with a visible fibrous mat, left. After treatment and separation can be seen of Figure 4.5, right. The electrodes are then surrounded by a high viscosity Norland UV epoxy used to create a $1 \mathrm{~mm}$ deep well in which the electrolyte was then added and a glass slide was added on top with more UV epoxy sealing the device. 

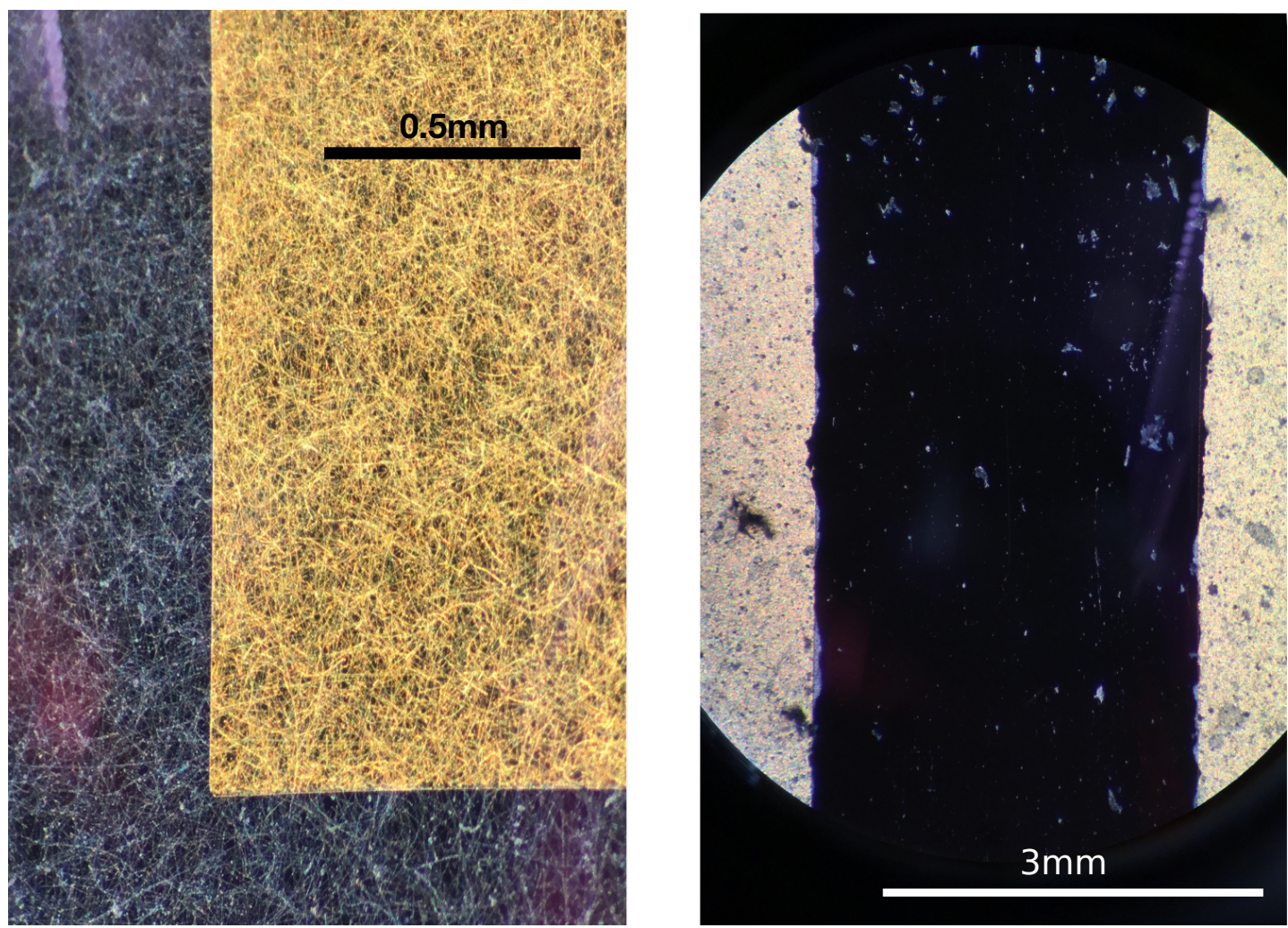

Figure 4.5: Optical microscope photos of the electrode seen in Figure 4.4 Left shows a close up of the edge of an electrode after deposition immediately after deposition. Right shows an optical microscope photo of space between the electrodes after post processing and being mechanically separated. The exposed thermal oxide is nearly black due to the highly reflective nature of the surface. 


\section{Chapter 5}

\section{Electrospun PEDOT:PSS/PVA/PEO EC}

\section{Performance Analysis}

\subsection{Introduction}

The initial EDLC performance assessment was to confirm charge storage behaviour and to identify the characteristics to be optimized. The experiments here were conducted using the $3 \mathrm{X}$ test device, as these results were for initial exploratory work. Three experiments were conducted: CV, CDC, and EIS. The testing was conducted using: a Kiethley 236 source measurement unit (SMU), an Agilent 33250A Arbitrary Waveform Generator, and an Analog Discovery 2 to aid in automation of EIS testing. The approach was to first investigate the electrochemical behaviour of the device with CV. With an operational voltage identified, DC charging is conducted to extract the maximum charge storage of the device. Finally, EIS is used to investigate and to separate kinetic electrochemical mechanisms within the EC. The EIS results are analyzed through the use of complex non-linear data fitting to an equivalent circuit model for EDLCs. All testing was conducted in a standard laboratory environment at room temperature with a relative humidity of $25 \%$. No special precautions for light, heat, or humidity were taking during operation. 


\subsection{Cyclic Voltammetry: Method and Result}

\subsubsection{Experimental Setup}

The initial charge storage characteristics of the EDLC were extracted through the measurement of the current as a linearly changing voltage is cycled from $0 \mathrm{~V}$ to $0.9 \mathrm{~V}$. Additionally, cyclic voltammetry will expose any electron-transfer mediated chemical reactions. The EDLC was attached to the Keithley 236. Each EDLC device was tested at 4 different voltage sweep rates for 50 cycles per trial. The different sweep rates help provide insight to the time-dependant characteristics for the EDL formation that is accentuated by a porous electrode.

\subsubsection{Results}

The initial test was to determine the electrochemical behaviour of the device through $\mathrm{CV}$ and the possible influence of the apparatus. In Figure 5.1, a 3X structure without ES PEDOT:PSS fibres was cycled to $0.9 \mathrm{~V}$ demonstrating the rectangular shape of a conventional capacitive storage device. The $0.9 \mathrm{~V}$ CV was selected to compare against existing PEDOT:PSS electrodes [1]. The $\mathrm{Au}$ electrode does demonstrate a slight peaking around $0.7 \mathrm{~V}$ which isn't uncommon in electrolytic solutions. But as seen in Figure 5.2, if the Au contact was in full contact with the electrolyte during operation, the contribution to the CV curve is extremely small. With the control measuring an areal capacitance of $76 \mathrm{nF} \mathrm{cm} \mathrm{cm}^{-2}$ compared to $4.5 \mu \mathrm{F} \mathrm{cm}^{-2}$ of the PEDOT:PSS/PVA/PEO EDLC results shown in Figure 5.2 . 


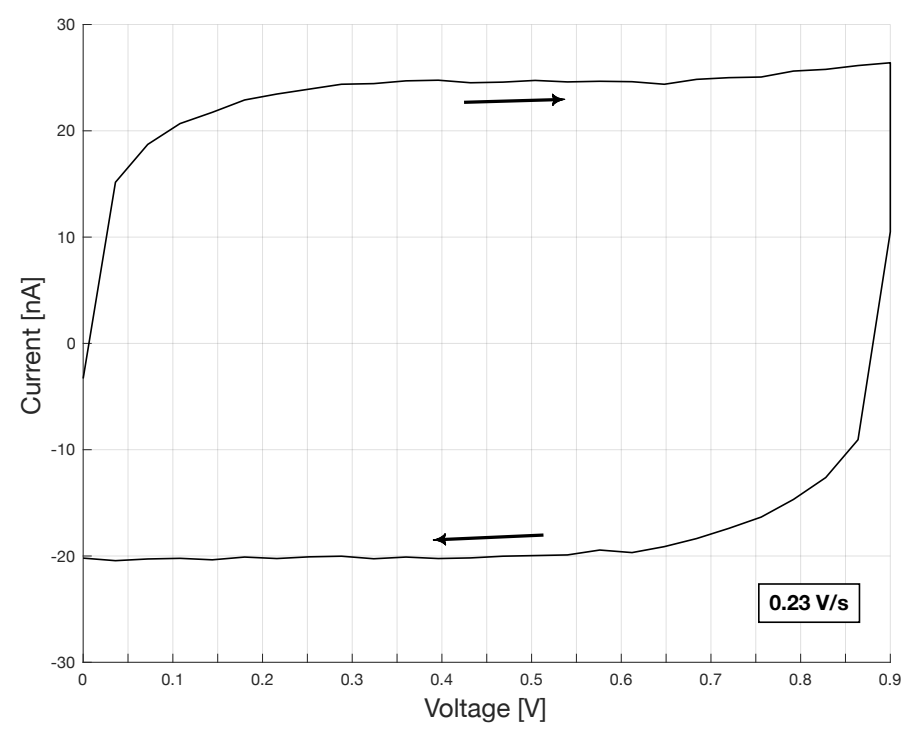

Figure 5.1: CV results of the control wafer. The arrows indicate the direction of the voltage sweep.

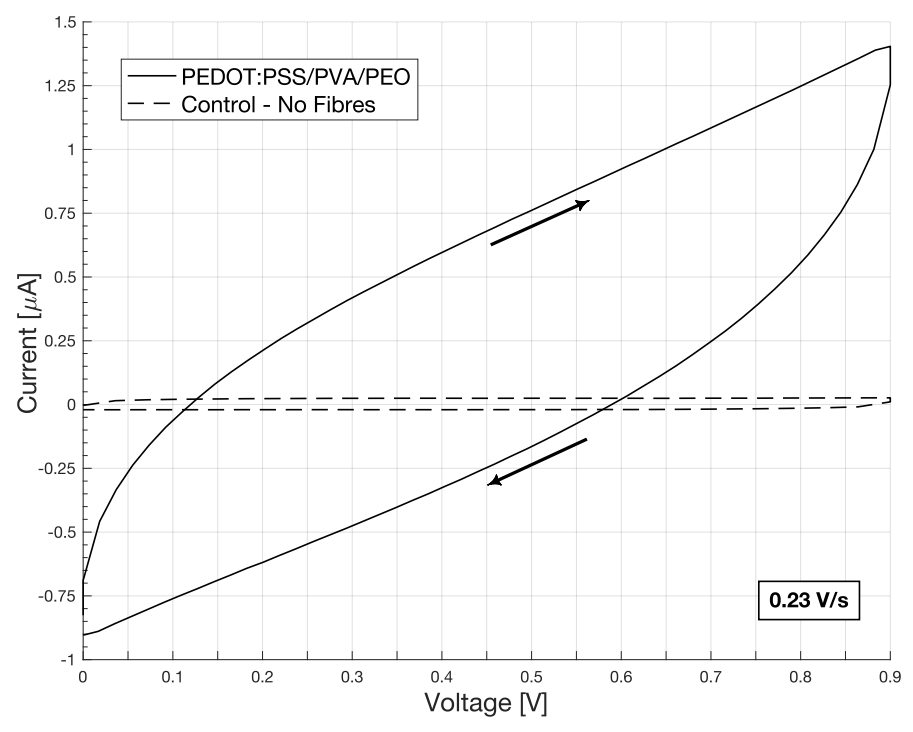

Figure 5.2: CV results of the ES PEDOT:PSS/PVA/PEO electrode, solid line, and the control device CV presented in Figure 5.1. The arrows indicate the direction of the voltage sweep.

The CV shape seen in Figure 5.2 is characteristic of PEDOT:PSS film devices and porous electrodes due the restriction to mass-transfer effects [38]. The next investigation was for operational voltage for this the EDLC was subject to a sequential voltage sweep of $0.9 \mathrm{~V}, 1.1 \mathrm{~V}, 1.8 \mathrm{~V}$, and $2.1 \mathrm{~V}$. A sweep rate around $0.2 \mathrm{~V} / \mathrm{s}$ was selected as this rate is slow enough to allow for possible redox reactions 
and ion intercalation to be present while being fast enough to possibly allow for reversibility 28], [41]. Due to the possible destructive nature of this test, devices tested above $1.1 \mathrm{~V}$ were not used for any further tests. The results of the PEDOT:PSS/PVA/PEO device at a range of voltages can be found in Figure 5.3 .

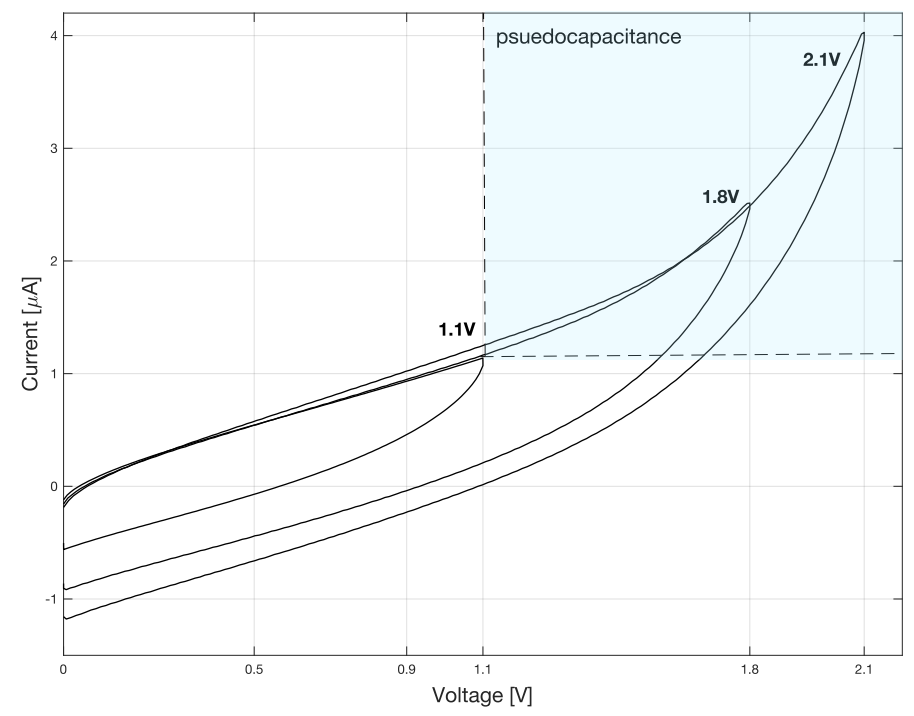

Figure 5.3: CV results of the EDLC for different maximum voltages. This determines the upper bound of the voltage window for EDLC vs pseudocapacitance operation.

The CV results confirm a voltage window of $0.9 \mathrm{~V}$, equivalent to that of a state-of-the-art PEDOT:PSS EC [14]. The CV additionally shows that the energy storage remains dominantly capacitive up to $1.1 \mathrm{~V}$. With an operational window voltage resolved, the sweep rates effect on capacitance can be determined, seen in Figure 5.4 over a host of sweep rates. Using equations 2.16 and 2.17. the capacitance and energy stored in the device is approximated. The integration of the CV curve was conducted numerically with MATLAB. 


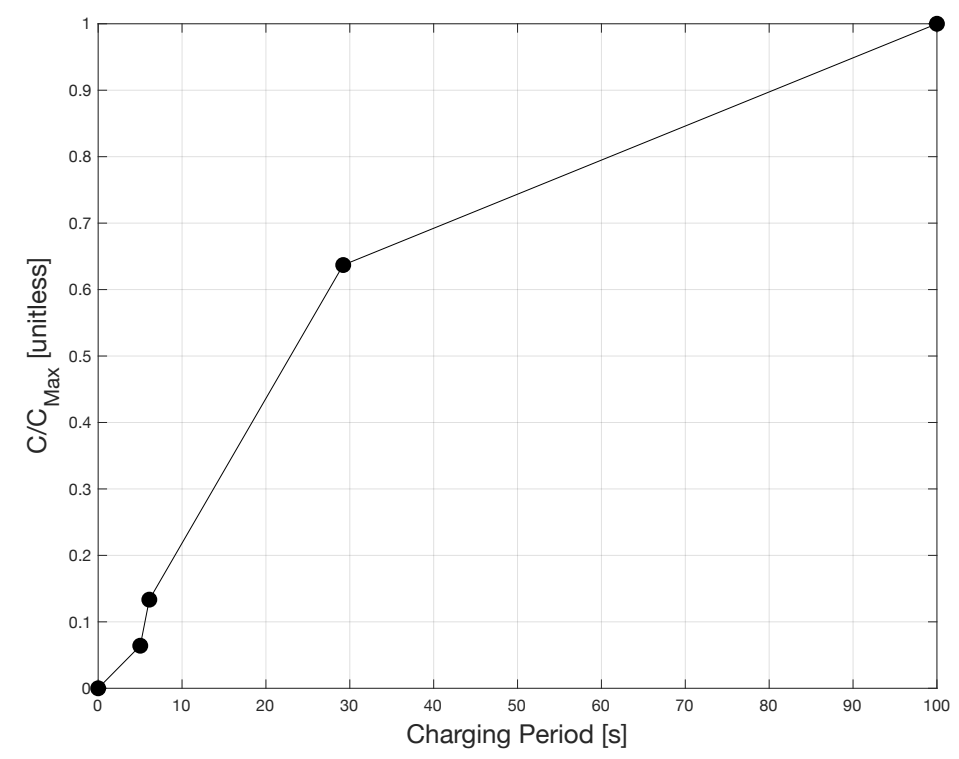

Figure 5.4: CV results at different charging periods (1/Sweep Rate). The connecting lines is for clarity of trend.

From these results, the capacitance was determined using Equation 2.16. The results from the CV testing are summarized in Table 5.1 below. Seen are the capacitance and energy density demonstrating a significant improvement compared to the control. This confirms that the presence of the ES PEDOT:PSS fibres are contributing to the energy storage via non-electrochemical means, when operating below $1.1 \mathrm{~V}$.

Table 5.1: Table of calculated CV results Sweep rate is $34 \mathrm{mV} / \mathrm{s}$

\begin{tabular}{cccc} 
Voltage Window $[\mathrm{V}]$ & Capacitance $\left[\mu \mathrm{F} \mathrm{cm}{ }^{-2}\right]$ & Energy $\left[\mu \mathrm{J} \mathrm{cm}^{-2}\right]$ & $\mathrm{C} /$ Control \\
\hline \hline 0.9 & 14.95 & 6.22 & 196.5 \\
\hline 1.1 & 16.08 & 8.64 & 211.3 \\
\hline
\end{tabular}

\subsection{Charging: Experimental Setup and Results}

\subsubsection{Experimental Setup}

A potentiostatic and galvanostatic charging of the EDLCs was conducted on the samples to extract the maximum charge storage capability by providing the time to saturate the EDL and other pos- 
sible charge storage mechanisms. The EDLCs were attached to a Keithley 236 SMU which sourced contant DC voltage of $1.1 \mathrm{~V}$ and measured the current being supplied by the positive terminal for up to 30 seconds. To determine a capacitance, Equation 2.12 was used and the integrated charge was calculated for current limited devices devices to limit missed current.

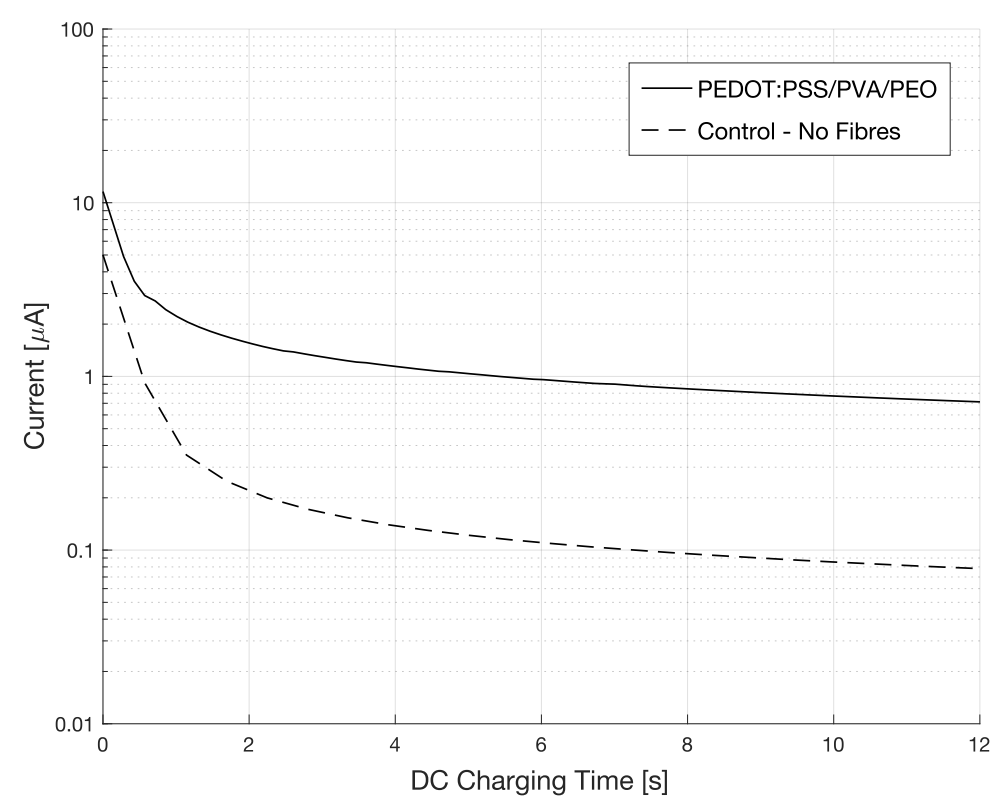

Figure 5.5: Plot of the ES PEDOT:PSS/PVA/PEO electrode and an uncoated control EDLC's current response to a step voltage, where $V\left(t=0^{-}\right)=0 \mathrm{~V}$ and $V\left(t=0^{+}\right)=1.1 \mathrm{~V}$.

The DC charging exponential decay curve that is characteristic of capacitive devices was still observed. The outline of the technique and analysis is described in Section 2.5.2. The results of the DC-charging tests are plotted in Figure 5.5. With the control plotted in addition for comparison. The expected decay of the current is observed under the potentiostatic stress.

It can be seen that the starting $I_{0}$ is less than the $I_{0}$ for the PEDOT:PSS/PVA/PEO, this is unexpected as Au on electrolyte should be more conductive and therefore lower DC resistance should correspond to a higher current by Ohm's law. After further investigation of the testing it was found that there is a small delay between the application of the voltage and the first measurements. By operating the SMU at the highest data rate, it was found the minimum delay for the initialization measurement was observed to uncontrollably fluctuate from a minimum of $114 \mathrm{~ms}$ to a maximum 
of $\approx 400 \mathrm{~ms}$. Due to this issue RC time-constant analysis was not used to find the capacitance of the $C_{d l}$ and the resistance of the substrate, electrode and electrolyte will be found through EIS in the following section, Section 5.4. The charge stored was found through numerical integration of the DC charging curves, executed numerically via MATLAB Table 5.2. The series resistance of the device will be investigated further using EIS.

Table 5.2: Table of calculated DC results for $V_{S}=1.1 \mathrm{~V}$.

\begin{tabular}{ccc} 
Capacitance $\left[\mu \mathrm{F} \mathrm{cm}^{-2}\right]$ & Charge $\left[\mu \mathrm{C} \mathrm{cm}^{-2}\right]$ & Energy $\left[\mu \mathrm{J} \mathrm{cm}^{-2}\right]$ \\
\hline \hline 119.5 & 107.6 & 48.46
\end{tabular}

With potentiostatic testing complete, the galvanostatic testing was run by applying a constant current to the device until a compliance limited maximum voltage was achieved, $0.9 \mathrm{~V}$ for these tests. Then the current's direction is flipped and the device is then discharged by constant drawing of the current out of the device. Figure 5.6 shows the EDLC device discharged at $5 \mu \mathrm{A}$ and the uncoated control discharged at $1 \mu \mathrm{A}$, the differing charge rates were done for scaling as a larger current drained the control device charge too fast for proper data collection with the apparatus.

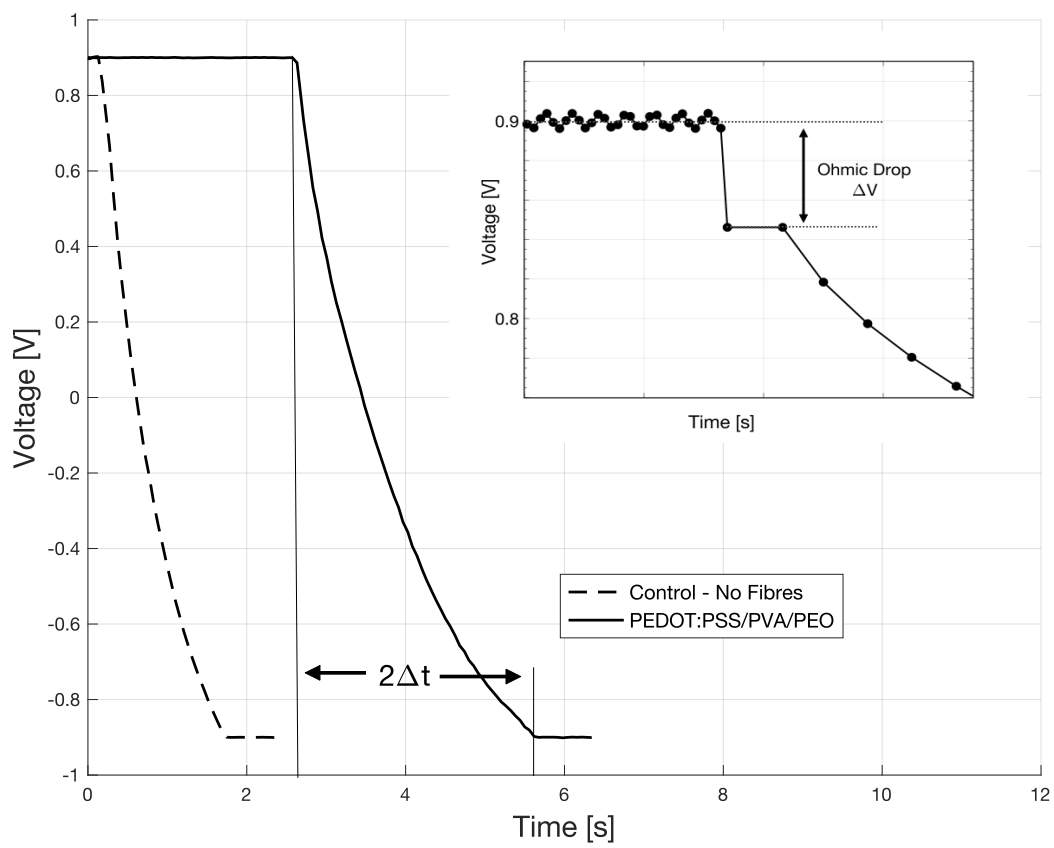

Figure 5.6: Plot of CCD of PEDOT:PSS/PVA/PEO electrode and an uncoated electrode. 
The inset on Figure 5.6 shows the ohmic drop exhibited from a constant current being removed from the device. This is often an ohmic drop caused by the electrical series resistance (ESR) of the EDLC. The ESR is found from the voltage drop simply through Ohm's law using the change in voltage over the change in current for the discharge region. For the PEDOT:PSS/PVA/PEO was found to be $10.8 \mathrm{k} \Omega$. This large amount of ESR found with such a small voltage difference would allow the contact resistance could be dominate at these measurements. The ESR usually primarily consists of the electrode's bulk resistance, electrolyte resistance, the wiring, and interconnects. In addition, the PEDOT:PSS/PVA/PEO and Au contact resistance will act as a non dissapative, voltage drop. This voltage drop is current independent and may dominate the small drop observed. Unfortunately the contact resistance of this ES material is not known. Given the charged state of the device the double layer and other EC phenomenon could be contributing to the ESR. 


\subsection{Electrochemical Impedance Spectroscopy of PEDOT:PSS/PVA EC}

\subsubsection{Experimental Setup}

To understand the EDLC electrodes and characteristics of the PEDOT:PSS more intimately, this work uses the frequency domain to dissect the impedance seen by the device, as outlined in Section 2.6. The EDLCs were attached in series with a $47.03 \Omega$ reference resistor and then attached to the Wavefunction generator and oscilloscope of an Analog Discovery 2 with high impedance probes in the configuration seen in Figure 5.7 allowing for $V_{21}$ and $V_{32}$ to be determined and from there the impedance of the DUT using Ohm's law. The impedance of each measurement was sampled multiple times and digitally averaged. Trials were conducted from $20 \mathrm{MHz}$ to $0.5 \mathrm{~Hz}$.

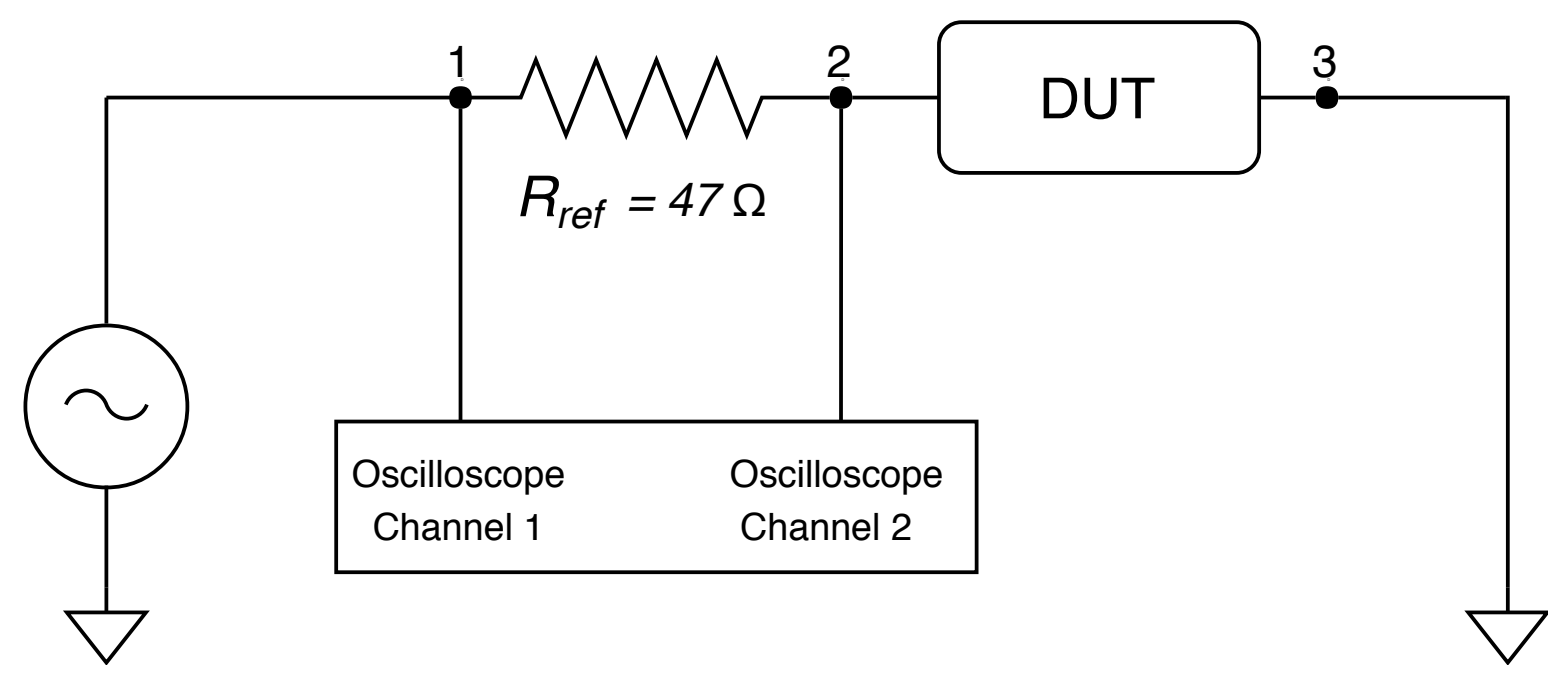

Figure 5.7: Schematic of the EIS illustration. The voltage is measured from 1-2 and 3-2. From here impedance of the DUT can be found with respect to the $47 \Omega$ reference resistor, R. 
Each device was first sampled from $25 \mathrm{MHz}$ to $1 \mathrm{MHz}$ to determine the $Z_{\text {real }}=0$ point in the high frequency range. Above this frequency the system will exhibit an inductive impedance due to the inductance in the wires and connections of the test apparatus 38. The intercept frequency will act as the starting HF point for the tests of the device then sweep down to $0.5 \mathrm{~Hz}$.

\subsubsection{EIS Results}
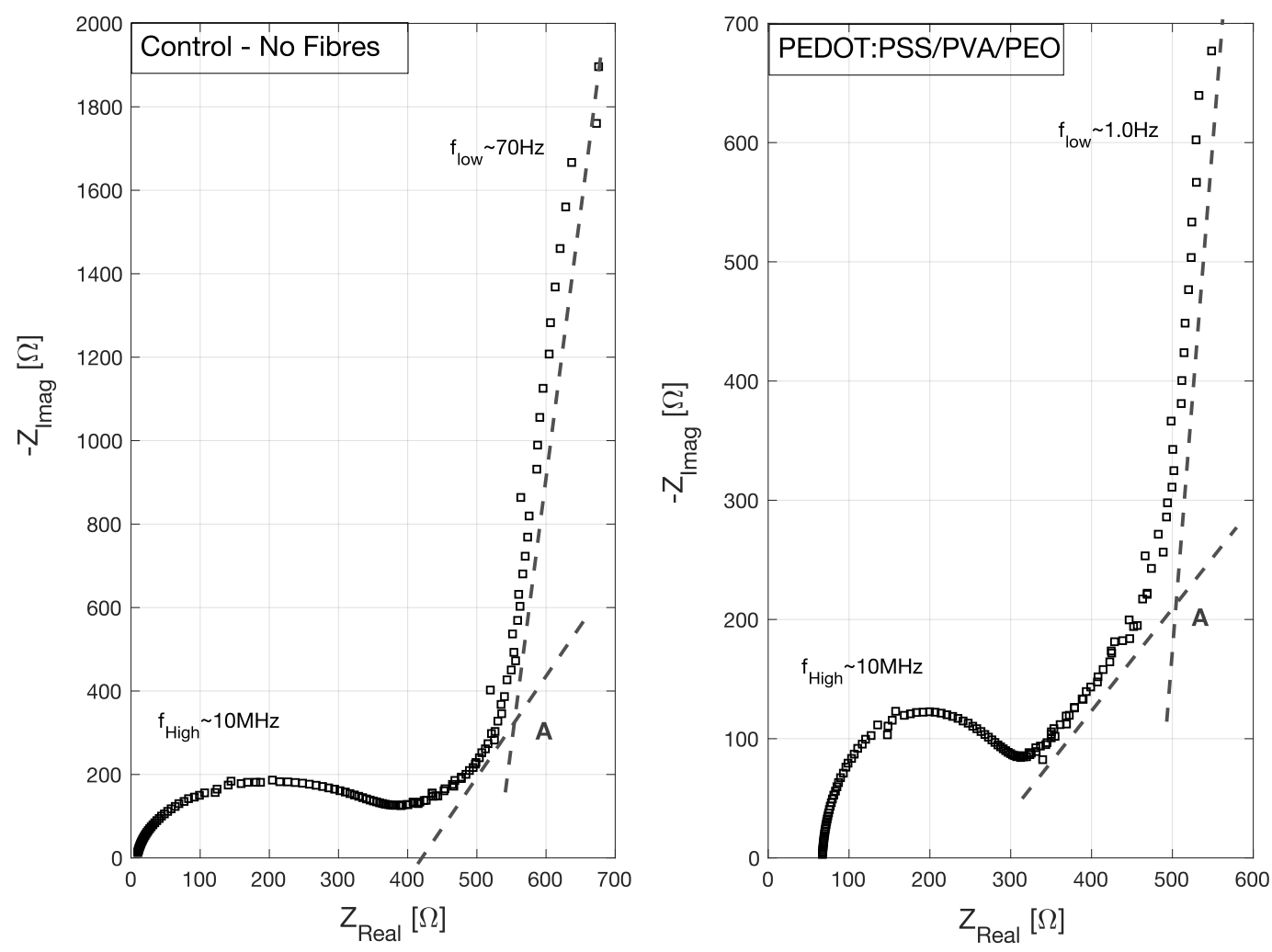

Figure 5.8: Nyquist plots for the EIS results of an uncoated control test structure and the PEDOT:PSS/PVA/PEO coated electrode.

The EIS of the control, left, and the PEDOT:PSS/PVA/PEO EDLC, right, is presented in Figure 5.8. The control data is shown to exhibit the common traits of a pure EDLC. Analyzing the control plot from left to right, we start at the high frequency region. The plot begins at $(9.86 \Omega,-12.96 i \Omega)$ extending into a clear RC semi-circle, indicative of the bulk electrolyte capacitance [38, 39. This region is followed by a small curve with no visible characteristic $45^{\circ}$ warburg diffusion flat, labelled A. For a device consisting of simply electrolyte and a planar electrode the presence of a small diffu- 
sion impedance (often a product of porous electrodes) is to be expected. The low frequency region shows a linear shape characteristic of the creation of the double layer.

For Figure 5.8 right, the PEDOT:PSS/PVA/PEO EDLC, starts at $Z_{\text {real }}=67.12 \Omega$ the presence of all the traits of the control exist with the addition of a clear Warburg diffusion region, labelled A. In addition, the EDL is only dominating the impedance spectrum around $300 \mathrm{~Hz}$ compared to around $10^{3} \mathrm{kHz}$. To further the analysis a complex non-linear data fit to an appropriate equivalent circuit model will be conducted.

\subsubsection{Equivalent Circuit Modelling}

An ECM was used to distill the relative magnitudes of the different impedance mechanisms. First, an appropriate model was selected based off of existing literature [37], 39] to capture the impedance characteristics of a porous electrode with non-faradaic charge storage. A qualitative investigation of the Nyquist plots seen in Figure 5.8 will provide insight to the dominance of diffusion impedance and electrode and substrate resistance. A proposed model which experimental data and mechanisms align with the devices can be found in figures 2.11 and 2.10 presented by 37]. The control plot's EIS data doesn't match the distinct complexity seen in the porous electrode devices [37, 38, 45]. The model was first investigated using ideal components without the CPEs. The model failed to fit the experimental data. The CPE replacement of the capacitors allowed for the successful fitting of the model by at the cost of possible excess complexity and reduced physical analogy due to the wide variety of interpretations of the CPE's meaning. An alternative to possibly capture the CPE's effects could be to explore transmission line ECM models. These can be used to capture the shape of Nyquist plots of porous electrodes but their use in modeling organic materials exhibiting ion intercalation is sparse. This is discussed further in Section 7.3.

Fitting data to an equivalent circuit model poses a few challenges: a large number of continuous variables creates a large solution space that can be computationally impractical to search to completion; The models will have a host of local minimas within the solution space in which simplistic complex-non-linear least squares algorithms can become trapped, making the results extremely de- 
pendent on the initial values and the search bounds; high variable models may contain redundant values or the creation of multiple similar solutions may present itself. These problems are not overcome by conventional data fitting software and methods [39], 42], [43]. To approach this issue two conventional optimization techniques were used symbiotically within a MATLAB script developed internally. The system consists of a genetic algorithm (GA) to search the solution space with a minimum of 5000 generations at a population of 1000. The normalized residual was used to assess the quality of the GA fit which resulted in the $5 \%$ of the population being kept for the next generation and a $10 \%$ mutation rate (Using both random mutation and cross-over). This was used to generate lists of approximate solutions to be conventionally fit by using trust-region reflective and Levenburg-Marquandt algorithms (LMA) [39], [43]. The fitness of the function was evaluated as the sum of square difference from the resultant plots. The fitness function looked at sum the difference from the fit data for both $Z_{\text {Real }}$ vs $f$ and $Z_{\text {Imag }}$ vs $f$. Using the ECM model presented in Figure 2.11 applying the porous electrode extension, replacing the capacitive elements with CPEs, provides the impedance equation for the DUT as:

$$
Z=R_{s}+\left[(j \omega)^{\alpha_{B}} Q_{B}+\left[R_{B}+\frac{1}{Q_{W} \sqrt{j \omega}} \operatorname{coth} \frac{\delta}{\sqrt{D}} \sqrt{j \omega}+\left[(j \omega)^{\alpha_{\mathrm{H}}} \mathrm{Q}_{\mathrm{H}}+\frac{1}{R_{i}+\frac{1}{(j \omega)^{\alpha} \text { ads } Q_{\mathrm{ads}}}}\right]^{-1}\right]^{-1}\right]
$$

Equation 5.1 requires 11 variables to be fit, summarized in Table 5.4.3. Of these, the resistive components can be approximated from the Nyquist plot according different regions and intercepts [37]-[39], allowing for a significant reduction in the initial solution space. The resistor components are lumped to describe multiple phenomenon not separable by EIS as introduced in Section 2.6.5. $R_{S}$ accounts for the interconnect, electrolyte, contact, and electrode resistances; $R_{B}$ accounts for the resistance arising from bulk impedance phenomenon and the resistance of the electrolyte in the porous structure; $R_{i}$, the interface resistance, will include the charge-transfer, adsorption, and EDL resistances. The next parameters are the CPE components, these can first reduce the solution space by restricting all exponents between 1 and 0 as all data is assumed to not be inductive within the experimental region. The CPE coefficients encompass the same collection of phenomenon as their companion resistors, as for their interpretation the CPE allows for closer experimental matching 
of the low frequency phenomenon witnessed in porous electrode and charge intercalation [38], [46]. Both of these mechanisms will be present in an ES PEDOT:PSS based EC. Finally, the diffusion layer thickness, $\delta$, and the diffusion coefficient, $D$, are grouped for all fitting as simply B as they are not temporally separable by this Warburg model.

Table 5.3: Summary of the ECM parameters used to fit to the EIS data.

\begin{tabular}{cccc}
$\#$ & Parameter & Name & Units \\
\hline \hline 1 & $R_{s}$ & Substrate and Electrode Resistance & $\Omega$ \\
\hline 2 & $Q_{B}$ & Bulk Capacitance & $\mathrm{S} \mathrm{s}^{\alpha_{\mathrm{B}}}$ \\
\hline 3 & $\alpha_{B}$ & CPE Exponent & unitless \\
\hline 4 & $R_{B}$ & Bulk Resistance & $\Omega$ \\
\hline 5 & $Q_{W}$ & Warburg Coefficient & $S s^{\frac{1}{2}}$ \\
\hline 6 & $\frac{\delta}{\sqrt{D}}$ & diffusion thickness & $s^{\frac{1}{2}}$ \\
\hline 7 & $Q_{H}$ & EDL Capacitance & $\mathrm{S} \mathrm{s} \mathrm{\alpha}^{\alpha_{\mathrm{H}}}$ \\
\hline 8 & $\alpha_{H}$ & CPE Exponent & $\mathrm{unitless}$ \\
\hline 9 & $R_{i}$ & Interface Resistance & $\Omega$ \\
\hline 10 & $Q_{\text {ads }}$ & Adsorption Capacitance & $\mathrm{S} \mathrm{s}^{\alpha_{\mathrm{B}}}$ \\
\hline 11 & $\alpha_{a d s}$ & CPE Exponent & unitless \\
\hline & & &
\end{tabular}

The reduction in solution space by bounding and simplifying parameters can help the data fitting process significantly. The final concern with a complex model is the low sensitivity of experimental data to certain parameters based off the magnitude of the phenomenons present during testing. To address this the approach of running $30+$ separate data fits to generate a range of solutions. Unfortunately, due to the possibility of multiple minimas, the multiple modal solution can not be represented by a single distribution. This makes finding the error on a solution and the confidence of the known value extremely difficult find. To highlight this issue, Figure 5.9 presents a histogram of the solutions for each parameter normalized to their respective mean value, it is important to note the range of values on the x-axis per parameter. For a large number of parameters a clear narrow range exists for the solution. For some, orders of magnitude can exist between modes, this 
creates a large uncertainty in the solution while demonstrating the inability to use an average. This will be the justification for excluding certain values for comparison. The best fit results for both the control and the PEDOT:PSS/PVA/PEO EDLC are seen in Figure 5.10.
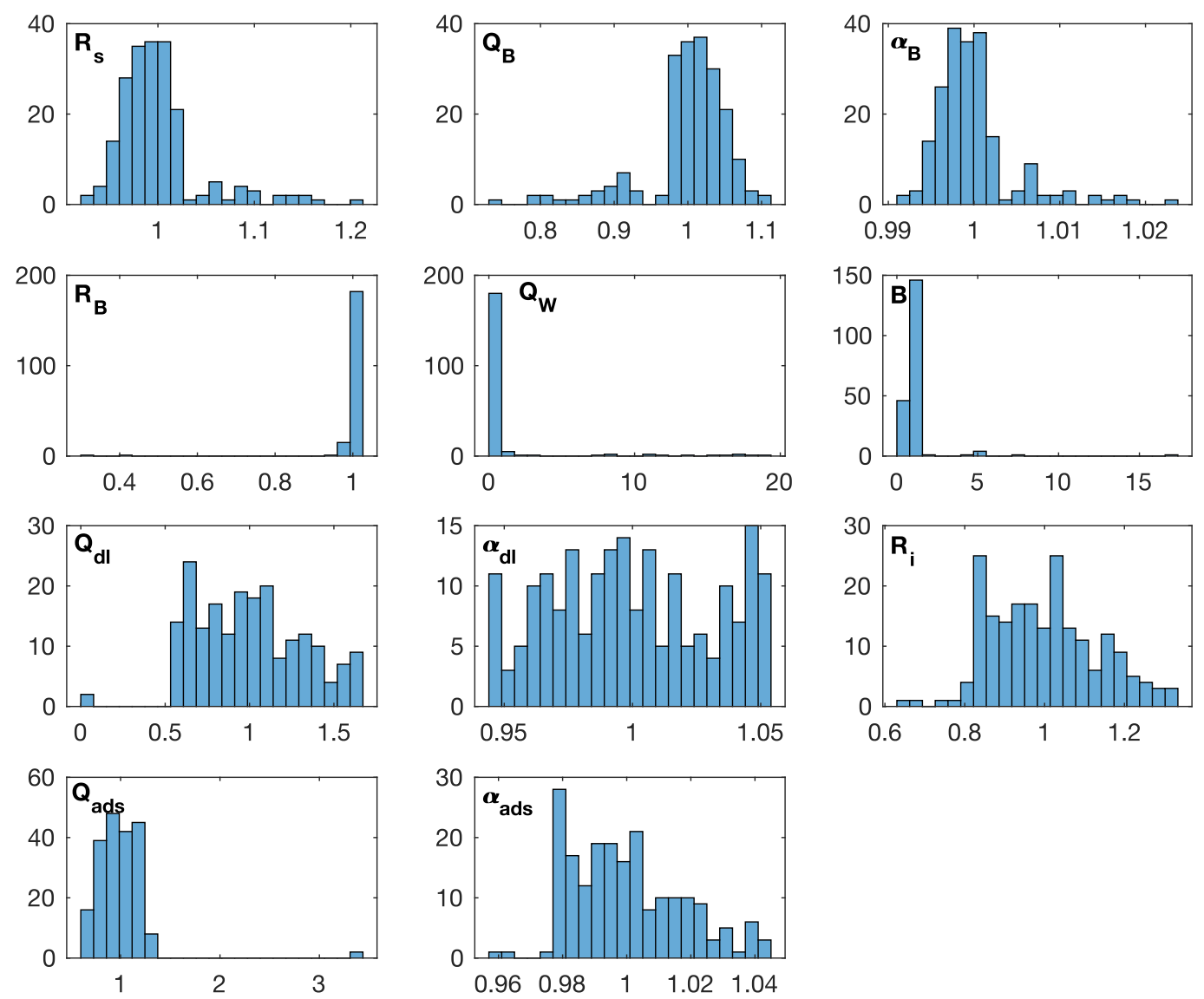

Figure 5.9: Visual representation of the spread of solutions for the control fit for 200 different simulations through GA and LMA all which met the residual criterion of the result. The $\mathrm{x}$ axis is the value/mean to show the $\%$ deviation of the data fitting result.

From the fit data seen in Table 5.4 below, the substrate resistance, $R_{S}$, of the PEDOT:PSS/PVA/PEO electrode shows a significant increase compared to the control. This demonstrates the resistance added by the ES mat due to the large resistance the fibres have. The $R_{S}$ seen by the control would be from the interconnects, wires and the bulk electrolyte resistance. The overall reduction in CPE coefficients confirms the presence of intercalation and charge transfer effects 46. The reduction of $R_{B}$ could hint at the improved conductivity of the electrolyte with PEO and PVA slowly dissolving 
in the presence of PC. In addition, thin film $\mathrm{CP}$ and non uniform surfaces are used to reduce the bulk resistance within an electrochemical system by breaking the uniformity of the double layer. Finally, a 2 orders of magnitude increase in $Q_{H}$ would corroborate the presence of a larger surface area and more EDL formation with the ES fibre mat.
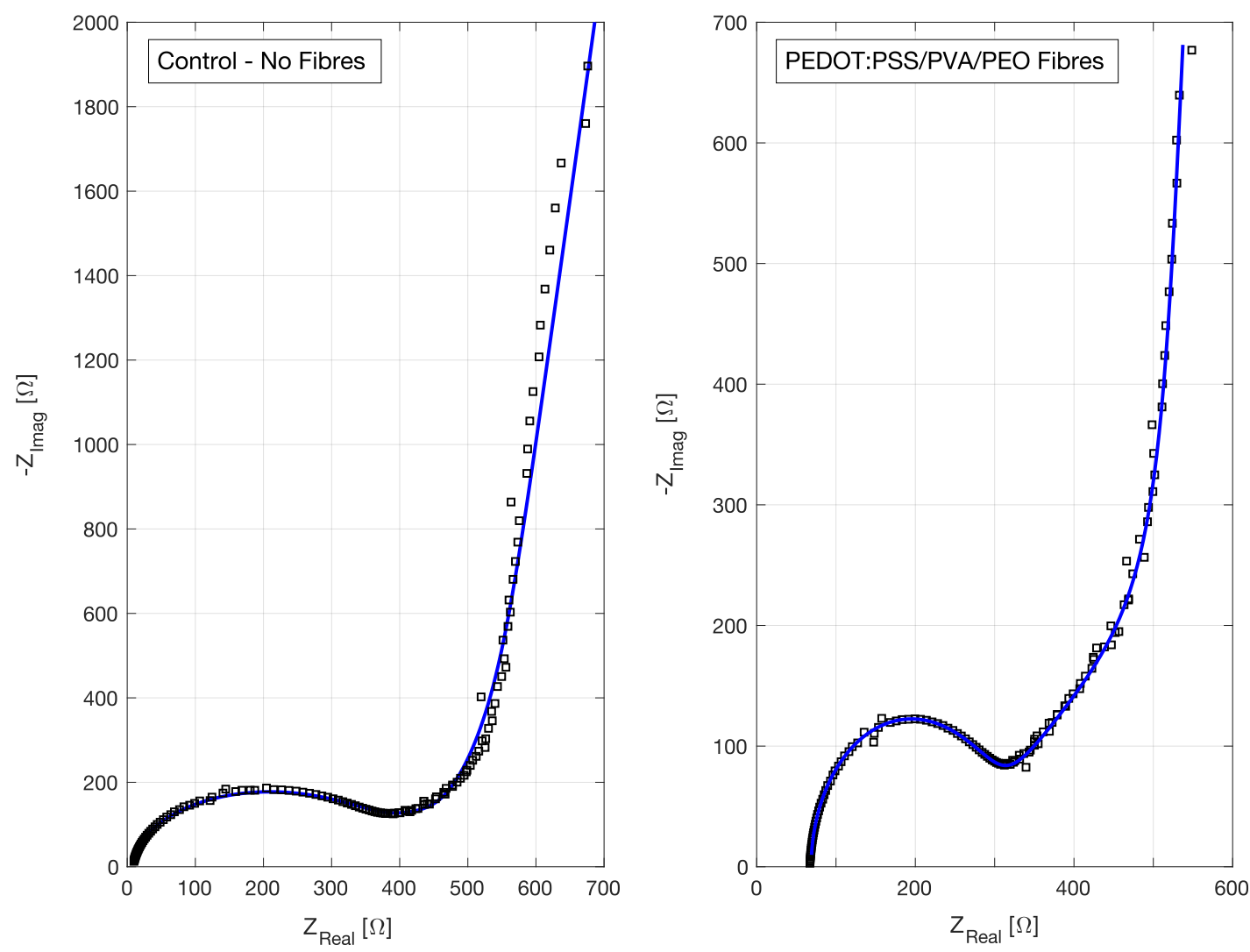

Figure 5.10: EIS of control and PEDOT:PSS/PVA/PEO with ECM data fitting results. 
Table 5.4: ECM fit parameters for control and PEDOT:PSS/PVA/PEO

\begin{tabular}{cccccc} 
& \multicolumn{2}{c}{ Control } & & \multicolumn{2}{c}{ PEDOT:PSS PVA PEO } \\
\cline { 2 - 3 } \cline { 5 - 6 } Parameter & Best Fit & Range $($ min - max $)$ & & Best Fit & Range (min - max $)$ \\
\hline \hline$R_{S}[\Omega]$ & 8.36 & $8.00-8.69$ & & 69.1 & $68.8-69.2$ \\
\hline$Q_{\mathrm{B}}\left[\mathrm{nS} \mathrm{s}^{\alpha_{\mathrm{h}}}\right]$ & 12.6 & $12.0-16.4$ & & 16.5 & $16.2-16.5$ \\
\hline$\alpha_{B}$ & 0.963 & $0.953-0.964$ & & 0.997 & $0.997-1$ \\
\hline$R_{B}[\Omega]$ & 374 & $374-392$ & & 237 & $236-241$ \\
\hline$Q_{\mathrm{W}}\left[\mathrm{mS} \mathrm{s}^{\frac{1}{2}}\right]$ & 0.253 & $0.203-0.436$ & & 2.9 & $2.9-10$ \\
\hline$B\left[\mathrm{~s}^{\frac{1}{2}}\right]$ & 0.0425 & $0.0005-0.2688$ & & 0.002 & $0.0007-0.002$ \\
\hline$Q_{\mathrm{H}}\left[\mu \mathrm{S} \mathrm{s}{ }^{\alpha_{\mathrm{H}}}\right]$ & 0.132 & $0.132-0.00146$ & & 12.8 & $7.06-12.9$ \\
\hline$\alpha_{\mathrm{H}}$ & 0.884 & $0.871-0.946$ & & 0.689 & $0.689-0.753$ \\
\hline$R_{i}[\Omega]$ & 343 & $325-460$ & 413 & $384-414$ \\
\hline$Q_{\mathrm{ads}}\left[\mu \mathrm{S} \mathrm{s}{ }^{\alpha_{\mathrm{ads}}}\right]$ & 0.586 & $0.544-0.958$ & 4.24 & $3.33-4.24$ \\
\hline$\alpha_{\mathrm{ads}}$ & 1 & $0.947-1$ & 0.995 & $0.968-1$ \\
\hline
\end{tabular}

\subsection{Summary}

This chapter presented first the initial proof of concept of an ES EC using a PEDOT:PSS/PVA/PEO solution. Through $\mathrm{CV}$ results there was an $\approx 200 \mathrm{X}$ increase in the measured capacitance after the addition of the fibres, resulting in an areal capacitance of $119.5 \mu \mathrm{F} \mathrm{cm}^{-2}$ corresponding to $0.0130 \mu \mathrm{Wh} \mathrm{cm}^{-2}$. This is to be expected as the porous high surface area of the fibre mat allow for the generation of more EDL compared to a planar Au electrode this is corroborated by the EIS results for $Q_{H}$, the range of solutions for the control are 2 orders of magnitude less than that of the PEDOT:PSS/PVA/PEO EC. Another confirmation to the choice of EIS model is the presence of a reduced CPE coefficient compared between the control and the fibres, $\alpha_{H}$. Literature suggests the CPE's physical interpretation represents the reduction of real resistance at low frequency, in a 
PEDOT:PSS polymer system this is caused by an increase of conductivity due to ion intercalation at low frequency which is present in the PEDOT:PSS/PVA/PEO system and not the control [46].

The ES EC demonstrated an approximate $R_{S}=70 \Omega$ compared to the control resistance of $R_{S}=8 \Omega$. This is a significant amount of added resistance. The difference, $\approx 62 \Omega$, which can be isolated as primarily series resistance of the ES fibres. If a transmission line model of the capacitor is used the extremely high resistance of the fibres would lead to a voltage drop through the length of a fibre. As voltage is dissipated through the electrode the surface area of the fibres will be less effective at storing charge.

The key findings from this iteration are: first, the non-faradaic operation up to $1.1 \mathrm{~V}$, higher operational voltages remain possible with the organic electrolyte but the charge storage could be dominated by pseudocapacitive mechanisms. Second, the CV of the ES EC has significantly deviated from the ideal capacitor rectangle shape seen by the control as the same charging rate. Finally and most importantly: the need to lower the series resistance of the fibres. This needs to be fixed to allow for increased thickness scaling and to improve the functionality of the material present,and reduce internal resistance power dissipation. This was approached in Chapter 3 during the ES optimization by increasing the mass fraction of PEDOT:PSS to the carry polymer (PEO) and by finding a suitable post treatment to improve PEDOT:PSS conformation and fibre-to-fibre contact, allowing for shorter paths through the normal of the electrode. 


\section{Chapter 6}

\section{Electrospun PEDOT:PSS/PEO EC}

\section{Performance Analysis}

\subsection{Introduction}

A limitation discussed in Chapter 5 was the difficulty consistently treating the fibres by conventional methods. A post-processing treatment would allow for significant improvements on ES PEDOT:PSS conductivity on par with literature [24], 25]. This chapter will summarize the EC performance analysis of the ES PEDOT:PSS/PEO/DMSO, outlined in Section 3.5, which provided fibres with drastically decreased as-spun resistance. The fibres were used for the devices tested in this chapter. For the remainder of the work, the ES PEDOT:PSS/PEO devices tested will simply be referenced to as the electrolytic capacitors, ECs. These were tested using the same techniques as Chapter 5 with the addition of the different sized test structures of Chapter 3: 1X, 3X, and 5X, to be referenced as $\alpha, \beta$, and $\gamma$ respectively. Chapter 6 finishes with a final iteration of the EC electrode. The ES PEDOT:PSS/PEO fibres were given 5 times the electrospinning deposition time, resulting in more ES fibres collected upon the surface. 1

\footnotetext{
${ }^{1}$ The thickness of the depositions were not found during the time of the writing. The relative thickness to depositions was gauged through optical attenuation of the Au electrodes through the ES fibre mat.
} 


\subsection{Cyclic Voltammetry: PEDOT:PSS/PEO}

As in Chapter 5, we begin with an electrochemical assessment of the electrode/electrolyte system through CV, seen in Figure 6.1 . We see pseudocapacitive effects beginning around $1.1 \mathrm{~V}$ which steadily increase with an increasing applied potential. The CV was conducted with a single $\gamma$ device as the geometry does not influence the presence of redox reactions.
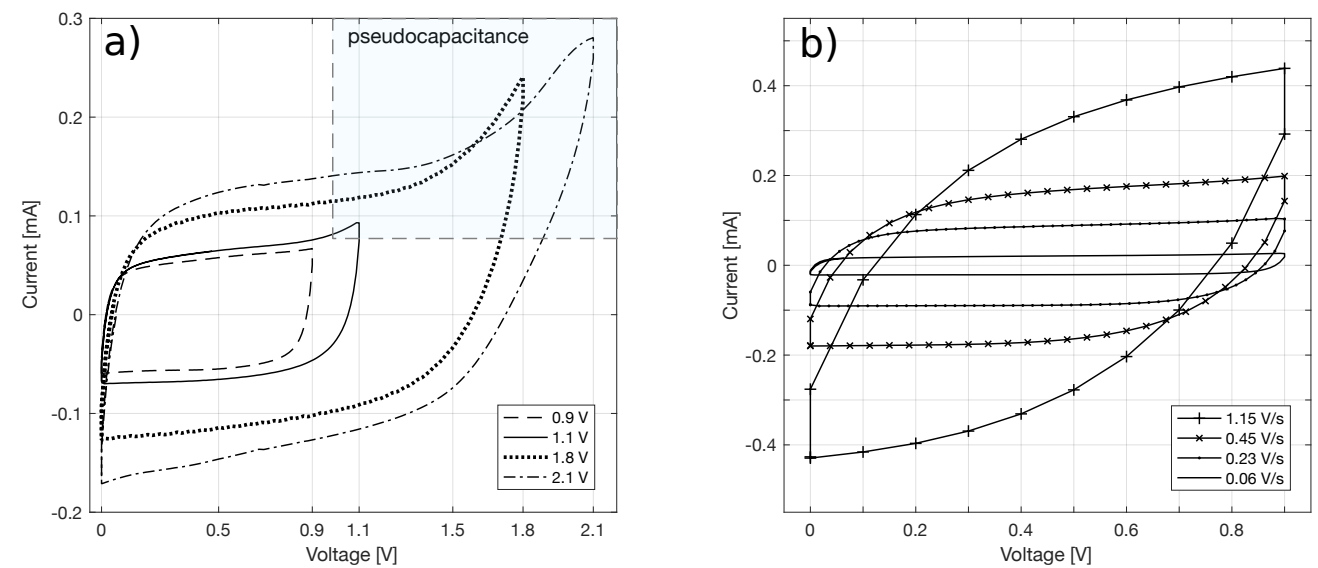

Figure 6.1: CV voltage and sweep rate results for $\gamma$. (a) we see the different voltage CV's at a sweep rate of $0.2 \mathrm{~V} / \mathrm{s}$ demonstrating the dominance of possible redox reactions at wider voltage windows. (b) the CV results for an EC subject to a sweep rate from $0.06 \mathrm{~V} / \mathrm{s}$ up to $1.15 \mathrm{~V} / \mathrm{s}$ demonstrating the deviation from the ideal rectangular shape.

With nearly pure non-faradaic operation at $0.9 \mathrm{~V}$ and nominal hybrid operation observed up to $1.1 \mathrm{~V}$, the sweep rate dependence was investigated to characterize the EDLC capacitance formation rate. This was conducted with all device sizes. Figure $6.1 \mathrm{~b}$ shows the charging progression at 4 different sweep rates. The $\mathrm{CV}$ characteristic goes from the rectangular shape at $0.06 \mathrm{~V} / \mathrm{s}$ to the diagonal shape at $1.15 \mathrm{~V} / \mathrm{s}$. Figure 6.2 shows the $\mathrm{CV}$ cycle period vs the areal capacitance characteristics of the three different sizes of devices. 


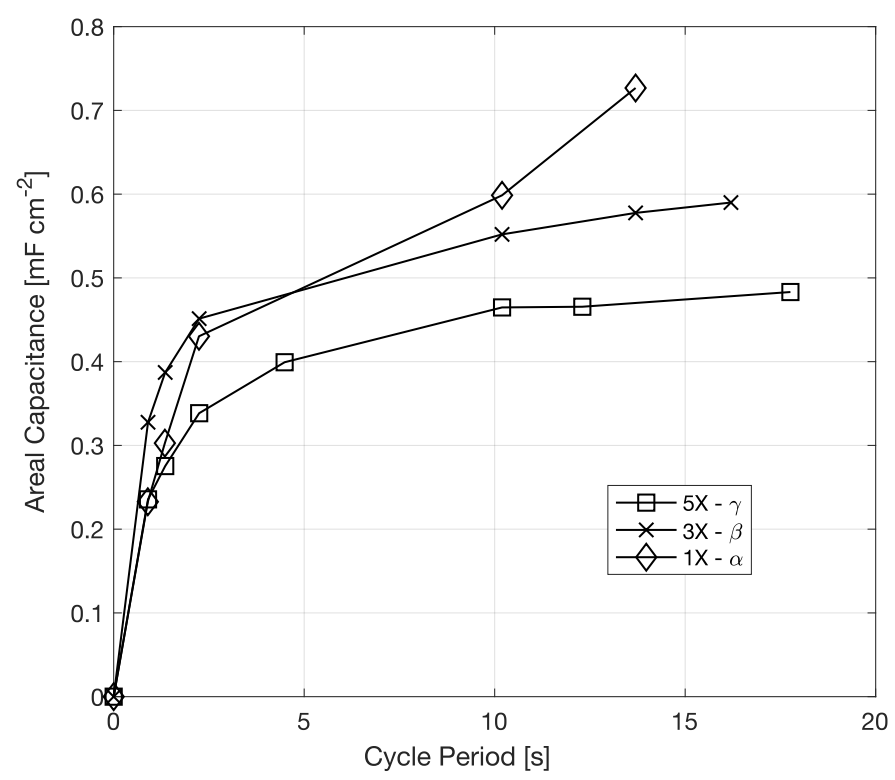

Figure 6.2: CV cycle period vs areal capacitance of three different sizes of PEDOT:PSS/PEO ECs

The three devices show similar charging rates with differences beginning to show after $10 \mathrm{~s}$. Here the $\alpha$ size shows increasing areal capacitance, higher than that of the larger devices. At the shorter cycle periods this could be due to the speed of charging the EDL on the surface versus the bulk of the electrode. The edge diffuse layer would be able to form quicker than an EDL within the porous electrode. Due to the small sample size of devices the DC charging tests were needed to investigate this trend.

With the electrochemical operation of the ES PEDOT:PSS/PEO fibres confirmed to be primarily non-faradaic up to $1.1 \mathrm{~V}$, and the capacitive rectangular CV obtained, the DC charging characteristics were investigated.

\subsection{Charging: PEDOT:PSS/PEO}

The ECs were then attached to a Keithley-236 SMU for DC charge testing. A $1.1 \mathrm{~V}$ voltage was applied to the EC for 30 seconds. Figure 6.3 shows the current response results for the different EC electrode sizes, where $V\left(t=0^{-}\right)=0 \mathrm{~V}, I\left(t=0^{-}\right)=0 \mathrm{~mA}$ and $V\left(t=0^{+}\right)=1.1 \mathrm{~V}$. With a highest achieved energy storage of $0.762 \mathrm{~mJ} \mathrm{~cm}^{-2}$, seen in Table 6.1, the ECs are demonstrating a charge storage performance increase compared to the devices of Chapter 5. 


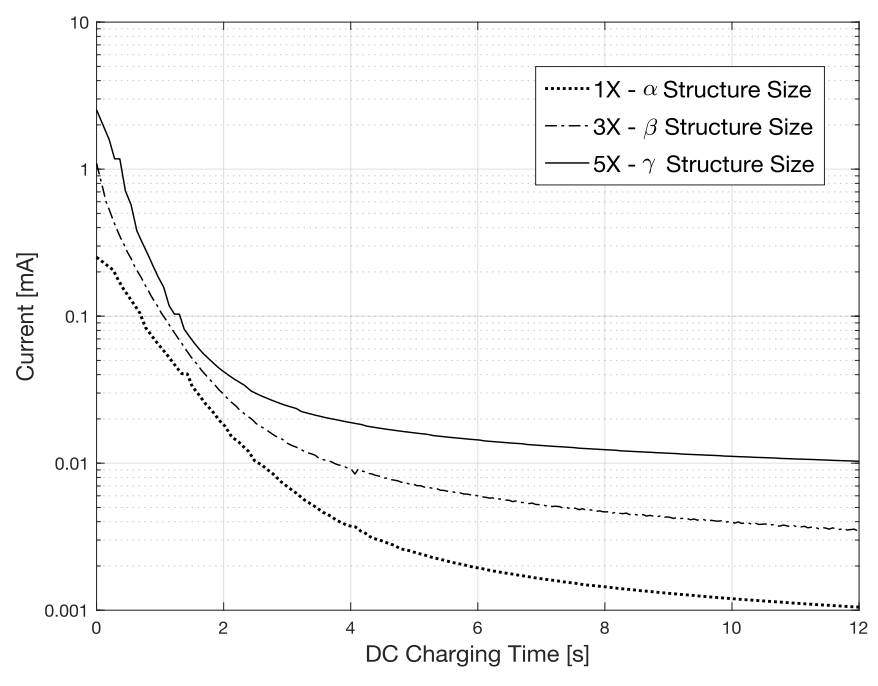

Figure 6.3: Plot of three different device sizes' current response to a applied step voltage, where $V\left(t=0^{-}\right)=0 \mathrm{~V}$, $I\left(t=0^{-}\right)=0 \mathrm{~mA}$ and $V\left(t=0^{+}\right)=1.1 \mathrm{~V}$.

A peculiar trend seen in these results is the overall increase in performance of the smaller device. A few possible factors contributing to this trend are:

- A smaller device requires less total ion motion to saturate generating larger capacitance over the charging time. This EDL saturation could occur faster in smaller devices. As the inner edge of the electrode will experience the largest electric field. This will drive the fastest motion of ions. The planar layout will lead to ion-ion interactions and shielding of the further ions.

- Edge effects: The 3 different devices have different bulk volume:surface area. The edge of the electrode could allow for a longer uninterrupted diffuse region to form. The contribution to the capacitance would be larger in smaller devices. This trend may still elicit an effect with respect to surface area of the bulk:perimeter. The structures $\alpha, \beta$, and $\gamma$ have approximate perimeter:surface area ratios of 11, 5, and 4 respectively. This doesn't reveal direct relationship except that the highest perimeter per area device demonstrated the highest areal capacitance. This could be component of the scaling discrepancy.

- A final influence to this effect could be the non-uniformity in the ES films. ES fibres are deposited as spots centered on electrostatically preferential points (Sharp points, shortest distance, energetically favorable, etc). Despite visual investigation showing highly uniform 
optical attenuation and depositions positioned to deposit centrally upon the electrodes, the larger contacts would have the larger deviation in uniformity. This possible non-uniformity would cause device size inconsistencies in the amount of active material on the electrodes.

Table 6.1: Summary of the calculated DC charging results at $V_{S}=1.1 \mathrm{~V}$. All values are divided by the surface area of the $\mathrm{Au}$ contact of their respective test wafers. $C / C_{i}$ is the ratio of the capacitance of the row specific $\mathrm{C}$ over the areal capacitance of the EC from chapter 5, ES PEDOT:PSS/PVA/PEO. The $\alpha$ structure produced the highest areal charge density, capacitance and energy densities were found to be $1.39 \mathrm{mC} \mathrm{cm}^{-2}, 1.26 \mathrm{mF} \mathrm{cm}^{-2}, \mathrm{and}_{0.762 \mathrm{~mJ}} \mathrm{~cm}^{-2}$ respectively.

\begin{tabular}{ccccc} 
Size & $\begin{array}{c}\text { Charge } \\
{\left[\mathrm{mC} \mathrm{cm}^{-2}\right]}\end{array}$ & $\begin{array}{c}\text { Capacitance } \\
{\left[\mathrm{mF} \mathrm{cm}^{-2}\right]}\end{array}$ & $\begin{array}{c}\text { Energy } \\
{\left[\mathrm{mJ} \mathrm{cm}^{-2}\right]}\end{array}$ & $C / C_{i}$ \\
\hline \hline$\alpha$ & 1.39 & 1.26 & 0.762 & 10.54 \\
\hline$\beta$ & 1.07 & 0.975 & 0.590 & 8.15 \\
\hline$\gamma$ & 0.983 & 0.898 & 0.541 & 7.48 \\
\hline
\end{tabular}

\subsection{Electrochemical Impedance Spectroscopy}

\section{of PEDOT:PSS/PEO EC}

The different structures were to provide insight to the scaling behavior of the fibre mat, but the different planar geometries will also create differences in the diffusion properties. These diffusion characteristics will appear in the mid to low frequency range on the EIS Nyquist shape. All three EIS results demonstrated proper qualitative trends, seen in Figure 6.4. The Warburg flat is most prevalent in the largest $5 \mathrm{X}-\gamma$ device while the smallest, $1 \mathrm{X}-\alpha$, shows only a $\mathrm{RC}$ semi-circle and double layer vertical, demonstrating the diffusion dominance in the larger devices. To investigate further, the curves were simulated and fit parameters were extracted. 

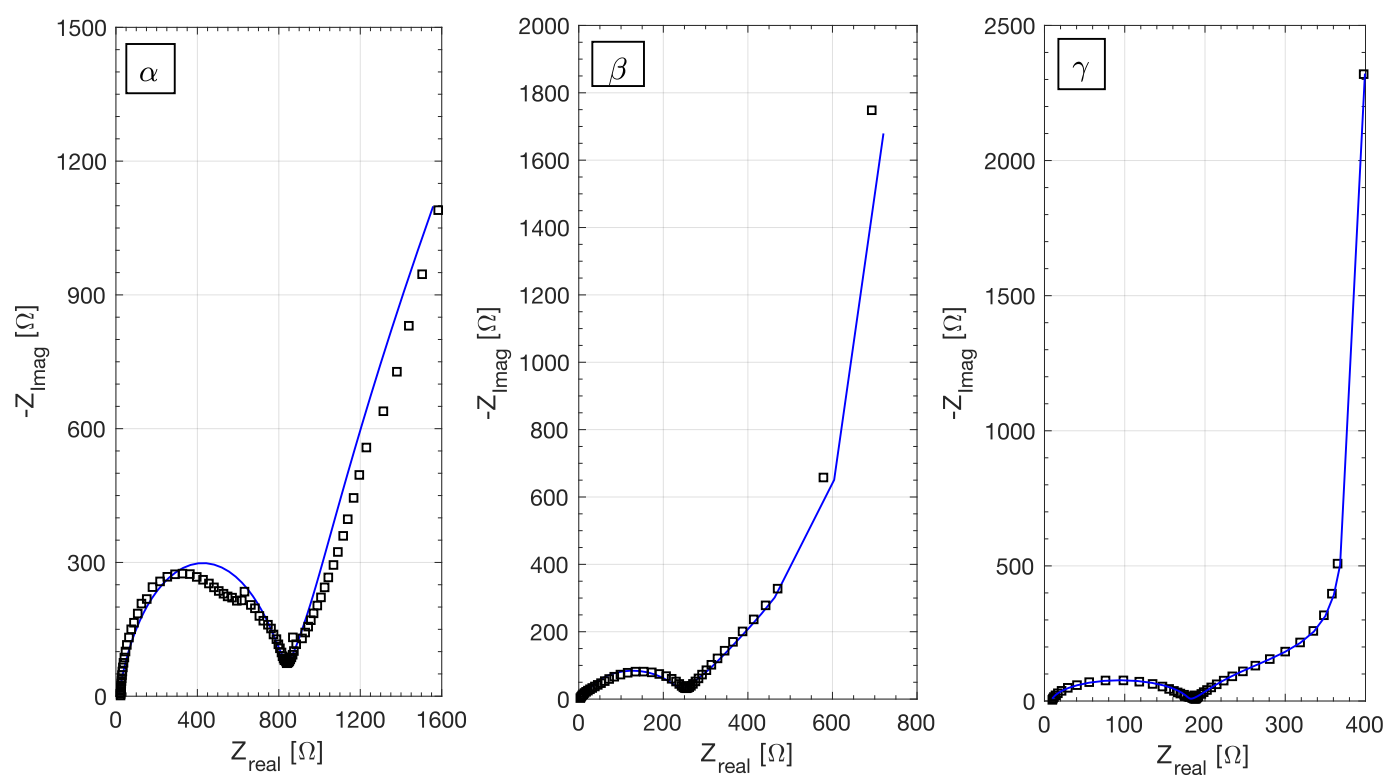

Figure 6.4: Experimental EIS results, black square points, and the ECM fit results, blue, for the 3 different sizes of structure for the EC from $\mathrm{MHz}$ to $0.5 \mathrm{~Hz}$.

The fit results can be seen in Table 6.2. Both $\beta$ and $\gamma$ showed reasonable agreement across most variables whereas for $\alpha$ the parameters $Q_{a d s}, R_{i}$, and, $\alpha_{a d s}$ were found to fluctuate wildly with little sensitivity or influence to the fit. This could be due to the $\alpha$ devices were found to demonstrate a large flattening within the semi-circular mid-frequency region. This observed flattening is sometimes qualitatively explained by two RC semi-circular phenomenon partially overlapping, as it can be seen as the sum of two offset semi-circles [38], [39]. The appearance of this separate semi-circle leads to significant difficulties in the data fitting process.

For higher confidence variables, $R_{S}$ demonstrates the expected inverse relationship with device size as current spreading would allow for reduced resistance through the bulk of the electrode, but overall a significant reduction in the $R_{S}$ is observed with respect to Chapter 5 results. This current spreading is also seen in the $R_{B}$ results as it drastically increases with device size. This characteristic is exacerbated by the planar geometry. Unfortunately, the disagreement of the other variables makes comparison of the device sizes difficult. The analysis of the remaining variables will be conducted in context of the other iterations in Chapter 6.5.2. 
Table 6.2: ECM fit parameters for ES PEDOT:PSS/PEO. The strange shape of the $1 \mathrm{X}$ results invoked in significant error during the fitting process due to the ECM mismatch. An extremely large deviation and multiple modes of solution occurred. $Q_{a d s}, R_{i}$, and $\alpha_{a d s}$ were found to fluctuate wildly and unexpectedly large or small values were appearing with little sensitivity or influence to the fit.

\begin{tabular}{|c|c|c|c|c|c|c|}
\hline \multirow[b]{2}{*}{ Parameter } & \multicolumn{2}{|r|}{$5 \mathrm{X}$} & \multicolumn{2}{|r|}{$3 \mathrm{X}$} & \multicolumn{2}{|r|}{$1 \mathrm{X}$} \\
\hline & Best Fit & Range (min - max) & Best Fit & Range (min - max) & Best Fit & Range (min - max) \\
\hline$R_{S}[\Omega]$ & 8.50 & $7.94-8.58$ & 11.5 & $9.25-13.1$ & 14.3 & $13.4-16.8$ \\
\hline$Q_{B}\left[\mathrm{nS} \mathrm{s} \mathrm{s}_{\mathrm{B}}\right]$ & 54.1 & $53.1-65.3$ & 728 & $320-738$ & 148 & $107.0-148.3$ \\
\hline$\alpha_{B}$ & 0.933 & $0.920-0.935$ & 0.801 & $0.779-0.810$ & 0.798 & $0.798-0.826$ \\
\hline$R_{B}[\Omega]$ & 170 & $169.9-173.2$ & 243 & $220-243$ & 803 & $795-819$ \\
\hline$Q_{W}\left[\mathrm{mS} \mathrm{s} \mathrm{s}^{\frac{1}{2}}\right]$ & 2.6 & $2.6-9.8$ & 0.65 & $0.65-82$ & 0.40 & $0.14-0.40$ \\
\hline$B\left[\mathrm{~s}^{\frac{1}{2}}\right]$ & 1.02 & $0.082-1.02$ & 1.38 & $0.004-1.42$ & 0.738 & $0.0188-0.738$ \\
\hline$Q_{H}\left[\mu \mathrm{S} \mathrm{s}^{\alpha_{\mathrm{H}}}\right]$ & 412 & $411-618$ & 133 & $133-676$ & 118 & $118-199$ \\
\hline$\alpha_{H}$ & 0.987 & $0.744-0.987$ & 0.970 & $0.604-1$ & 1 & $0.698-1$ \\
\hline$R_{i}[\Omega]$ & 205.9 & $205.9-274.2$ & 290. & $282-1887$ & 1450 & $318-46000$ \\
\hline$Q_{a d s}\left[\mu \mathrm{S} \mathrm{s}^{\alpha_{\mathrm{ads}}}\right]$ & 511 & $502.2-4100$ & 295 & $248-324$ & 79800 & $94.3-1.00 \times 10^{6}$ \\
\hline$\alpha_{a d s}$ & 0.992 & $0.986-1$ & 0.988 & $0.027-1$ & 0 & $0-1$ \\
\hline
\end{tabular}




\subsection{Long Deposition PEDOT:PSS/PEO EDLC}

\subsubsection{Introduction}

A significant motivation behind the use of ES was to investigate the scaling of the electrode thickness due to the sparse large pores present in ES mats. To investigate, the ES collection time was $5 \mathrm{X}$ that of all structures presented so far in all previous chapters. This thick EC structure will be referenced in this work as a TEC. For this test only, $\alpha$ size devices were made. The TEC on an $\alpha$ wafer covered with a thick deposition ES can be seen in Figure 6.5 below. Here we see significant build up of the ES PEDOT:PSS as the transparency of the film is significantly reduced, but the uniformity of the mat was also significantly impacted.

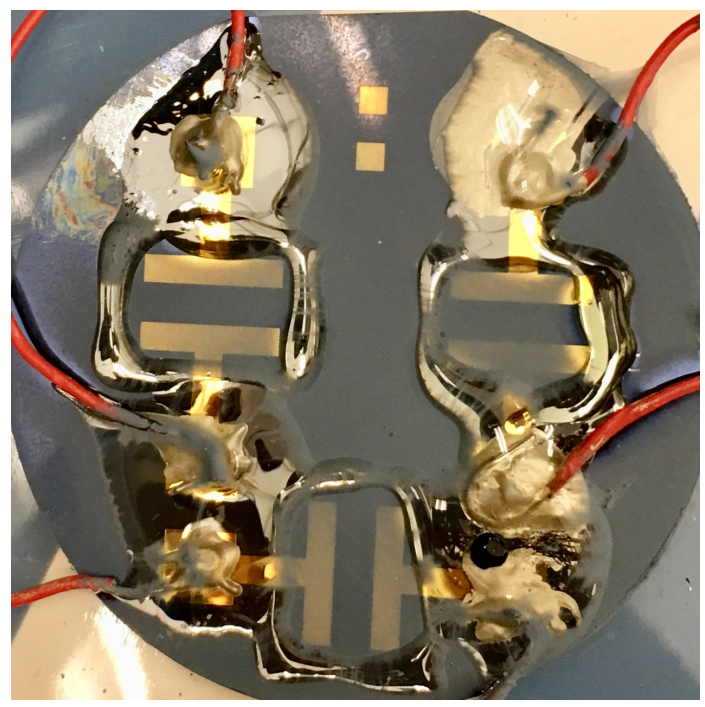

Figure 6.5: Photo of thick PEDOT:PSS deposition on $\alpha$ structure.

Post treatment remained the same for the polymer and it was subject to a few tests to find the performance of the device. The thick deposition of PEDOT:PSS/PEO was investigated for the capacitance, energy, lifecycle, and, the EIS spectrum was analyzed.

\subsubsection{Results}

The first test was to use constant current charge/discharge (CCD) to investigate the charging phenomenons while also investigating the areal power density, seen in Figure 6.6 a. For the CCD the 
TEC was subjected to $\pm 50 \mu \mathrm{A}$ until $\pm 1.1 \mathrm{~V}$ was measured across the DUT. The CCD testing should return a linear decay for an ideal capacitor but the TEC demonstrates a curved characteristic, as seen in Figure 6.6 3 . The extended charge time from $0.8 \mathrm{~V}$ to $1.1 \mathrm{~V}$ supports the presence of pseudocapacitance present in the CV results. The ES PEDOT:PSS/PEO fibres exhibit minor higher energy pseudocapacitive charging. Using $\Delta \mathrm{T}$ and $\Delta \mathrm{V}$ in Equation 2.12, the capacitance found through the constant current charge/discharge is $2.3 \mathrm{mF}$ and a full cycle average power density of $0.229 \mathrm{~mW}$.

Once again, DC charging conducted with an applied voltage of $1.1 \mathrm{~V}$, seen in Figure6.6b. Following the methods outlined in Chapter 5, using equations $2.12 \& 2.13$ the areal energy density was found to be $3.18 \mathrm{~mJ} \mathrm{~cm}^{-2}$ and the areal capacitance was $5.25 \mathrm{mF} \mathrm{cm}^{-2}$. Table 6.3 summarizes these results and has the DC charging results for the EC of the same size from earlier in Chapter 6 for comparison. We see that there is an $\approx 4.2 \times$ increase in the areal storage metrics.

Table 6.3: The calculated DC charging results for the TEC structure at $V_{S}=1.1 \mathrm{~V}$. The $\alpha$ EC DC results from Section 6.3 are added for comparison.

\begin{tabular}{cccc} 
Device & $\begin{array}{c}\text { Charge } \\
{\left[\mathrm{mC} \mathrm{cm}^{-2}\right]}\end{array}$ & $\begin{array}{c}\text { Capacitance } \\
{\left[\mathrm{mF} \mathrm{cm}^{-2}\right]}\end{array}$ & $\begin{array}{c}\text { Energy } \\
{\left[\mathrm{mJ} \mathrm{cm}^{-2}\right]}\end{array}$ \\
\hline \hline TEC & 5.8 & 5.25 & 3.18 \\
\hline$\alpha$-EC & 1.39 & 1.26 & 0.762 \\
\hline
\end{tabular}

With a few performance metrics found, the next step was to investigate the cycle life of this device. The testing used an Agilent 33250A Arbitrary Waveform Generator. A TEC was attached to the system and allowed to cycle from $1.1 \mathrm{~V}$ to $0 \mathrm{~V}$ at $f=0.2 \mathrm{~Hz}$ in 10,000 cycle increments. After an increment the device was attached to the Keithley $236 \mathrm{SMU}$ and a CV capacitance test was conducted at $0.23 \mathrm{~V} / \mathrm{s}$. 

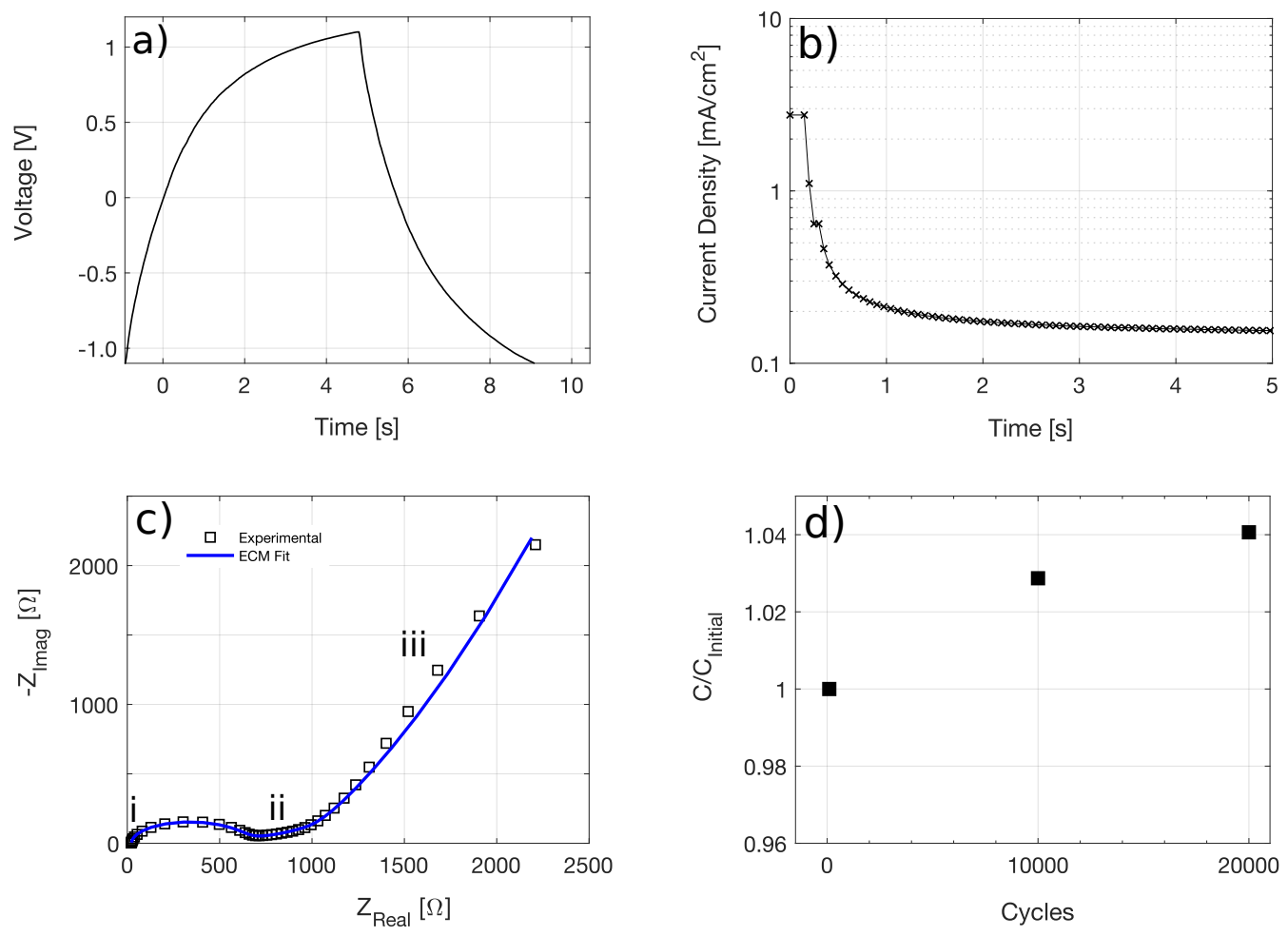

Figure 6.6: (a) constant current charge/discharge results at $0.050 \mathrm{~mA}$. (b) DC charging at $1.1 \mathrm{~V}$ fixed. The visible plateaus are from auto sweeping the current limit to reduce lost charge during the integration calculation. (c) Fitted nyquist plot of EIS results from $10 \mathrm{MHz}$ to $0.5 \mathrm{~Hz}$ demonstrating a conventional porous electrode EDLC. (d) lifecycle testing of the material showing a slowly increasing capacitance.

EIS was again used to determine diffusion properties and the resistance of the thicker electrode deposition. Figure 6.6. shows the experimental data and the ECM fit following the model and method outlined for all previous EIS results. A qualitative analysis reveals a substrate resistance of $\approx 20 \Omega$ and electrolyte bulk resistance of $\approx 550-600 \Omega$. A distinct feature is the low frequency region appearing to be dominated by the warburg $45^{\circ}$ slant appearing after a shallow flat. The observed shapes are first: a narrow diffusion limited region is seen at point iii on Figure 6.6. followed by the steadily increasing electrolytic double layer formation through the low frequency region. The high porosity and depth of the structure lead to these dominate low frequency shapes. The slanted low frequency diffusion layer is also characteristic of a non-reflective boundary condition Warburg element. In PEDOT:PSS Li-ion intercalation would allow for significant charge storage and a steady extended flow of ions forming a double layer as IHP ions would be intercalated [28]. The resistance through the double layer is still rapidly increasing [37], [38]. Applying the same data 
fitting technique as above Table 6.4 summarizes the values found.

Table 6.4: ECM Fit parameters for the thick deposition of PEDOT:PSS/PEO fibres.

\begin{tabular}{ccc} 
Parameter & Best Fit & Range \\
\hline \hline$R_{S}[\Omega]$ & 22.8 & $22.7-23.4$ \\
\hline$Q_{B}\left[\mathrm{nS} \mathrm{s}{ }^{\alpha_{\mathrm{B}}}\right]$ & 10.4 & $10.3-12.6$ \\
\hline$\alpha_{B}$ & 0.989 & $0.988-1.00$ \\
\hline$R_{B}[\Omega]$ & 573 & $572-630$ \\
\hline$Q_{W}\left[\mu \mathrm{S} \mathrm{s} \mathrm{s}^{\frac{1}{2}}\right]$ & 341 & $341-1430$ \\
\hline$B\left[\mathrm{~s}^{\frac{1}{2}}\right]$ & 1.23 & $0.818-1.27$ \\
\hline$Q_{H}\left[\mu \mathrm{S} \mathrm{s}^{\alpha_{\mathrm{H}}}\right]$ & 316 & $23.6-318$ \\
\hline$\alpha_{H}$ & 0.711 & $0.355-0.711$ \\
\hline$R_{i}[\Omega]$ & 557 & $226-1870$ \\
\hline$Q_{a d s}\left[\mu \mathrm{S} \mathrm{s}^{\alpha_{\mathrm{ads}}}\right]$ & 87.9 & $21.3-176$ \\
\hline$\alpha_{a d s}$ & 0.631 & $0.619-1.00$ \\
\hline
\end{tabular}

The data fit produced a few single mode localized solutions for parameters such as $R_{S}, Q_{B}, \alpha_{B}$, $R_{B}$, and B. Overall the nyquist plot of the TEC demonstrates a significant increase in $R_{S}$ increasing the maximum path length of current through PEDOT:PSS. The total real impedance seen at low frequencies is caused by a thicker porous layer and thus a bigger separation through the EDL to reach the Au contact. The most striking result is the change in shape of the Nyquist itself as a clear porous EDLC characteristic is seen with the prominence of a moderately shallow extended EDL formation layer, region iii. With a dominant diffusion slant present, the thick electrode device is a good fit for the ECM model selected, aligning with the expected phenomenon [20], [37].

The results of the life cycle testing are demonstrating a slow linear increase, seen in Figure 6.6 d. The increase is hypothesized to be the result of the cycling and prolonged exposure to the solvent. Over time the solvent could continually remove the PEO carrier polymer until saturation opening 
up more surface area within the mat, as seen previously in Figure 3.5. of Chapter 3. Removal of the PEO increases the PEDOT:PSS/electrolyte interface volume improving the energy storage potential of the fibre mats through:

- More direct conductor/electrolyte interface giving rise to a thinner Helmholtz layer and higher electric field at the surface.

- The improved access to PEDOT:PSS allows for more readily adsorbed and intercalated ions. This improves the charge storage and charging rate of the material.

The cycling itself could also enhance the effect as PEDOT:PSS does undergo a volumetric swelling during ion intercalation, this physical motion could also provoke the removal of PEO trapped in the fibres [55]. It would be assumed that this increase has a finite limit as only so much PEO can be removed and non-reversible reactions and other aging effects would begin to degrade the electrode.

\subsection{Conclusion}

The goal to decrease the resistance of electrode material and obtain a higher energy density electrode was successfully achieved through the use of PEDOT:PSS/PEO with suitable post-processing. For equivalent sized devices, the $R_{S}$ was reduced from $\approx 70 \Omega$, in the EC presented in Chapter 5, to about $\approx 11 \Omega$ while also allowing for faster charging rates, as seen by the more ideal $\mathrm{CV}$ shapes presented for $0.2 \mathrm{~V} / \mathrm{s}$ sweep rates. Furthermore, the EIS captured a significant increase in capacitance by an $\approx 10 \times$ increase around the fit values for $Q_{H}$ while also demonstrating the diffusion impedance's dominance in larger devices. Finally, a 5 times increase in deposition time corresponded closely with an increase in the capacitance and energy storage of the device. The primarily non-faradaic energy storage and fast rate of the device suggests valuable potential for an extremely long life time or for the potential of higher voltage operation for increased energy density. 


\section{Chapter 7}

\section{Conclusions}

\subsection{Summary of Results}

Three distinct stages of devices were made and tested within this thesis. In the first stage the initial proof of concept using an independently developed PEDOT:PSS/PVA/PEO solution. These fibres were then incorporated into an EC in which a high series resistance and low mat density significantly reduced the scaling potential of the material. These fibres demonstrated a two orders of magnitude increase of the capacitance and energy storage over an uncoated electrode. Stage 2 was to develop a higher purity PEDOT:PSS fibre using UHMW PEO and a post-processing treatment for ES fibre mat to increase density and conductivity. The solution consisted of Baytron $\mathrm{P} / \mathrm{PEO} / \mathrm{DSMO}$ in $93.55 / 1.45 / 5 \%$ by weight, resulting in a fibre with $46.5 \%$ PEDOT:PSS content before post-treatment using an ES apparatus with the range of parameters given in Table 7.1. These final fibres demonstrated resistances between 152 - $1370 \Omega /$ sq with an average resistance around $900 \Omega /$ sq across 15 measured devices demonstrating a 5 orders of magnitude decrease in resistance to the previous stage. The reduction of the resistance was due to the use of DMSO increasing fibre mat density and interconnection between fibres in addition to expected CEA effects. The ES and post-processing resulted in fibres with an average fibre diameter of $171 \mathrm{~nm}$.

Table 7.1: Electrospinning apparatus parameters for PEDOT:PSS/PEO solution for conductive nanofibres.

\begin{tabular}{ccc} 
Parameter & Lower Bound & Upper Bound \\
\hline \hline Needle Voltage & $5 \mathrm{kV}$ & $7 \mathrm{kV}$ \\
\hline Collector Voltage & $-2 \mathrm{kV}$ & $-4 \mathrm{kV}$ \\
\hline Spinning Distance & $12 \mathrm{~cm}$ & $14 \mathrm{~cm}$ \\
\hline Humidity & $25 \% \mathrm{RH}$ & $35 \% \mathrm{RH}$ \\
\hline
\end{tabular}


The new PEDOT:PSS fibres provided a $10 \times$ increase in energy storage performance metrics over the stage one results while reducing the resistance of the electrode. The final stage of development began on improving the areal capacitance and energy density by using longer deposition times to amass more material on the electrode. This resulted in a continued increase in energy storage demonstrating thickness scaling allowing for further improving of the areal capacitance. The brief look into scaling factors demonstrated that the smallest device size demonstrated only a $1.68 \times$ increase in resistance despite $5 \times$ decrease in size (inversely proportional to the potential current density) thus projecting a possible reduction in $R_{S}$ with smaller devices.

A summary of the EC storage metric can be found in Table 7.2. The stage 1 results, which were $\approx 200 \times$ the capacitance over an uncoated electrode, were improved by a factor of $10.9 \times$ in stage 2 . Stage 3 increased the storage metrics an additional $4.2 \times$. The final stage 3 electrode demonstrated an $\approx 10^{4}$ increase in storage over an uncoated electrode.

Table 7.2: Summary of the EC performance for all stages.

\begin{tabular}{cccc} 
Stage & $\begin{array}{c}\text { Charge } \\
{\left[\mathrm{mC} \mathrm{cm}^{-2}\right]}\end{array}$ & $\begin{array}{c}\text { Capacitance } \\
{\left[\mathrm{mF} \mathrm{cm}^{-2}\right]}\end{array}$ & $\begin{array}{c}\text { Energy } \\
{\left[\mathrm{mJ} \mathrm{cm}^{-2}\right]}\end{array}$ \\
\hline \hline 1 & 0.108 & 0.120 & 0.049 \\
\hline 2 & 1.39 & 1.26 & 0.762 \\
\hline 3 & 5.8 & 5.25 & 3.18 \\
\hline
\end{tabular}

The lifetime results for the ES PEDOT:PSS electrode material demonstrates a slow and steady increase in capacitance most likely caused by the electrolyte dissolving PEO and opening up pore structure and access to PEDOT:PSS. This lifetime improvement was observed over the first 20,000 cycles.

\subsubsection{Comparison to State-of-the-art Literature}

The initial stage of ES development corroborated with solution compositions for PEDOT:PSS electrospinning recently reported [25]. The ES fibres were advanced by depositing with a significantly higher molecular weight PEO. These simple fibres incorporated into an EC presented comparable 
metrics to more mature technologies and fabrication methods. The direct comparison to a PEDOT micro EC presented in [14] and seen in Table 7.3 with a collection of other mature state-of-the-art electrodes. The PEDOT micro EC does demonstrate a larger areal capacitance at $9 \mathrm{mF} \mathrm{cm}^{-2}$ relying on energy dense redox reactions for the storage at the cost of $10 \%$ loss of capacitance at 1000 cycles.

Table 7.3: The materials and performance metrics of thin-film microscale electrochemical capacitors. Where $C_{1000} / C_{\text {Initial }}$ is a brief measure of the degradation of the device after 1000 cycles.

\begin{tabular}{lccccc|} 
Material & $\begin{array}{c}\text { Capacitance } \\
{\left[\mathrm{mF} \mathrm{cm}^{-2}\right]}\end{array}$ & $\begin{array}{c}\text { Potential } \\
\text { Window }[\mathrm{V}]\end{array}$ & $\begin{array}{c}\text { Energy } \\
{\left[\mathrm{mJ} \mathrm{cm}^{-2}\right]}\end{array}$ & $\frac{\mathrm{C}_{1000}}{\mathrm{C}_{\text {Initial }}}$ & Ref \\
\hline \hline ES PEDOT:PSS & 5.25 & 1.1 & 3.18 & $\sim 100$ & - \\
\hline Graphene & 16 & 1 & 2 & $\sim 98$ & 12 \\
\hline PEDOT & 9 & 0.8 & 3 & $\sim 90$ & $\mid 14$ \\
\hline CNT & $<1$ & 0.9 & $<1$ & $\sim 100$ & 15 \\
\hline Graphene/PANI & 326 & 1 & 41 & $\sim 92$ & $16 \mid$ \\
\hline Graphite/Pt & 5.4 & 1 & 2.7 & - & 17 \\
\hline rGO & 0.4 & 0.8 & 0.144 & - & 18 \\
\hline Graphene & 6 & 1 & - & - & $13 \mid$ \\
\hline
\end{tabular}

Table 7.3 summarizes the similar in material thin film electrodes using mature fabrication techniques. Unfortunately, a persistent issue in this field of research is lack of established reporting metrics, were rarely does the research report the same performance metrics or report on physical aspects of the electrodes, such as thickness or mass [1]. This work presents the surface area normalized metrics, as the footprint in IC processing is an important parameter. The potential window, lifetime, and if the electrolyte use was aqueous or non-aqueous would all be additionally valuable metrics to allow for better cross material comparison. Here the success of being in a similar magnitude of leading technologies is encouraging for electrospinning's presence in the discussion of energy storage for autonomous sensors while not requiring aggressive post-processing such as carbonization or exotic materials. 


\subsection{Contributions to the Field}

- Independent replication and confirmation of ES PEDOT:PSS/PVA and ES PEDOT:PSS/PEO of the results presented in 25$]$.

- Presentation of electrospinning "troubleshooting" method to quickly explore and test different solutions with consistency while focusing on dominate apparatus and solution parameters.

- Dissemination of the early results at an international materials research conference MRS 2018.

- To be published: novel ES PEDOT:PSS/PVA/PEO solution and fibres. Novel ES PEDOT:PSS/PEO solution for higher fibre PEDOT:PSS content fraction with initial postprocessing method.

- To be published: Creation and investigation of novel ES PEDOT:PSS fibres in a non-aqueous electrolyte system developed on Si for microscale energy storage applications.

- The initial development of GA enhanced LMA and TRR data fitting techniques to allow for reduced initial condition and bound sensitivity.

- Testing of a modern porous electrode ECM [37].

\subsection{Future Work and Improvements}

The results of the thesis have demonstrated electrospinning's ability to fabricate an EC without the use of carbonization or other limiting post-processing. Future research to build on the successes, optimize the results, and work towards a fully realized IC device begins with:

- Electrospinning: Further increase the molecular weight of PEO and decrease the PEO concentration in the fibre. ES could be possible at significantly reduced concentrations using reduced spinning distance and voltage. Other polyelectrolyte and surfacant additives could allow for electrospinning at lower viscosity and lower carry polymer concentrations. 
- Electrospinning characterization: Finding a definitive deposition rate for the process and characterizing the thickness and distribution of the depositions would allow for vastly improved understanding of the electrodes being fabricated. Work with the transmission spectra and a prolifometer would offer insight to the physical metrics of the depositions.

- Electrospinning uniformity: Improving the uniformity of deposition through the investigation of the use of electrostatic lensing, multiple needles, larger collection plates or machine automated movement of the collection wafer.

- Electrospinning surface area characterization: Exploring Brunauer-Emmett-Teller (BET) theory to characterize the surface area of the ES material. A characterization of the exposed PEO and PEDOT:PSS regions will need to be conducted as well.

- Electrospinning: Incorporate or replace PEO with PVDF: on its own, PVDF is often used in the separator layers in Li-ion batteries as it has demonstrated an ability to improve lifetime through the reduction of different electrochemical fault mechanisms. The PVDF would also be a suitable candidate for a separator layer in a parallel-plate geometry.

- Post-Processing: Ideally a proper post-processing technique would remove all of the carrier polymer while maintaining the high porosity, fibre structuring and possible morphology benefits of the electrospinning deposition. Further investigation of different non-destructive soaking times and chemicals to remove PEO and allow for a proper non-destructive DMSO curing is needed.

- Electrode structure: With the electrode classified, other geometries must be investigated for proper on-chip integration. Two primary structures should be investigated: interdigitated electrodes and stacked planar electrodes. The former would be a simple implementation as no separator layer is needed and the PEDOT:PSS is patterned through a shadow mask processes or photolithography. The latter will allow for maximum areal energy density with the design hurdles of the fabrication of a suitable ES separator layer.

- Voltage: Due to the selection of an anhydrous electrolyte, a larger voltage window could be investigated while ensuring the occurring reactions are primarily reversible. This could increase energy density but may jeopardize lifetime. 
- Electrolyte: Additives and different electrolytes could offer improved performance in the PEDOT:PSS system. Given the current voltage operation, aqueous electrolytes have shown to vastly improve energy storage but with the risk of reducing lifetime.

- Gel Electrolyte: With the PEDOT:PSS/PEO deposition, the PEO can be seamlessly incorporated in a gel electrolyte system. Gel electrolytes often reduce energy and power density due to the reduce ion mobility and lower conductivity but offer significant advantages when packaging and sealing devices.

- Equivalent Circuit Model: Finding a reduced model that more elegantly captures the shape of the EIS of the PEDOT:PSS to add confidence to the values found. As well as improved data fitting to correctly isolate the values for each parameter.

- Equivalent Circuit Model: Establish an EDLC model to better describe the device for use by circuit and system designers to aid in possible use of the material in industry applications.

- Extension to Flexible Substrates: The results of these fibres show promise in EC, unlike the majority of microscale IC capacitors it is compared against. Electrospinning offers inexpensive expansion to larger surface areas and does not requires high resolution patterning or etching for the high surface area. A flexible substrate would allow for use in a host of other applications. 


\section{References}

[1] N. A. Kyeremateng, T. Brousse, and D. Pech, "Microsupercapacitors as miniaturized energystorage components for on-chip electronics", Nature Nanotechnology, vol. 12, no. 1, pp. 7-15, 2016. DOI: 10.1038/nnano.2016.196.

[2] F. Orfei and G. Orecchini, "Autonomous sensors: Existing and prospective applications", in ICT - Energy - Concepts Towards Zero - Power Information and Communication Technology. 2014. [Online]. Available: http://www.intechopen.com.

[3] J. F. M. Oudenhoven, L. Baggetto, and P. H. L. Notten, "All-solid-state lithium-ion microbatteries: A review of various three-dimensional concepts", Advanced Energy Materials, vol. 1, no. 1, pp. 10-33, 2010. DOI: 10.1002/aenm.201000002.

[4] S. G. Chalk and J. F. Miller, "Key challenges and recent progress in batteries, fuel cells, and hydrogen storage for clean energy systems", Journal of Power Sources, vol. 159, no. 1, pp. 7380, 2006. DOI: $10.1016 / \mathrm{j} \cdot$ jpowsour.2006.04.058.

[5] Battery Performance Characteristics, Accessed: 2018. [Online]. Available: https : / / www . mpoweruk.com/performance.htm.

[6] M. Aneke and M. Wang, "Energy storage technologies and real life applications - a state of the art review", Applied Energy, vol. 179, pp. 350-377, 2016. DOI: $10.1016 / \mathrm{j}$. apenergy . 2016.06.097.

[7] A. González, E. Goikolea, J. A. Barrena, and R. Mysyk, "Review on supercapacitors: Technologies and materials", Renewable and Sustainable Energy Reviews, vol. 58, pp. 1189-1206, 2016. DOI: $10.1016 /$ j.rser.2015.12.249.

[8] A. Ferris, S. Garbarino, D. Guay, and D. Pech, "3d ruo2microsupercapacitors with remarkable areal energy", Advanced Materials, vol. 27, no. 42, pp. 6625-6629, 2015. DOI: 10.1002/adma. 201503054 .

[9] L.-h. WANG, M. Toyoda, and M. Inagaki, "Dependence of electric double layer capacitance of activated carbons on the types of pores and their surface areas", New Carbon Materials, vol. 23, no. 2, pp. 111-115, 2008. DOI: 10.1016/s1872-5805(08)60015-3.

[10] J. Kang, S. Atashin, S. H. Jayaram, and J. Z. Wen, "Frequency and temperature dependent electrochemical characteristics of carbon-based electrodes made of commercialized activated carbon, graphene and single-walled carbon nanotube", Carbon, vol. 111, pp. 338-349, 2017. DOI: $10.1016 /$ j.carbon.2016.10.017.

[11] S. Ramakrishna, K. Fujihara, W.-E. Teo, T. Yong, Z. Ma, and R. Ramaseshan, "Electrospun nanofibers: Solving global issues", Materials Today, vol. 9, no. 3, pp. 40-50, 2006. DOI: 10 . 1016/s1369-7021(06)71389-x.

[12] J. Lin, Z. Peng, Y. Liu, F. Ruiz-Zepeda, R. Ye, E. L. G. Samuel, M. J. Yacaman, B. I. Yakobson, and J. M. Tour, "Laser-induced porous graphene films from commercial polymers", Nature Communications, vol. 5, no. 1, 2014. DOI: 10.1038/ncomms6714.

[13] D. Song, E. B. Secor, Y. Wang, M. C. Hersam, and C. D. Frisbie, "Transfer printing of sub-5 um graphene electrodes for flexible microsupercapacitors", ACS Applied Materials 63 Interfaces, vol. 10, no. 26, pp. 22 303-22 310, 2018. DOI: 10.1021/acsami.8b06235. 
[14] N. Kurra, M. Hota, and H. Alshareef, "Conducting polymer micro-supercapacitors for flexible energy storage and ac line-filtering", Nano Energy, vol. 13, pp. 500-508, 2015. DOI: 10.1016/ j.nanoen.2015.03.018.

[15] T. M. Dinh, K. Armstrong, D. Guay, and D. Pech, "High-resolution on-chip supercapacitors with ultra-high scan rate ability", J. Mater. Chem. A, vol. 2, no. 20, pp. 7170-7174, 2014. DOI: $10.1039 / \mathrm{c} 4 \mathrm{ta00640b}$.

[16] Z.-S. Wu, K. Parvez, S. Li, S. Yang, Z. Liu, S. Liu, X. Feng, and K. Müllen, "Alternating stacked graphene-conducting polymer compact films with ultrahigh areal and volumetric capacitances for high-energy micro-supercapacitors", Advanced Materials, vol. 27, no. 27, pp. 4054-4061, 2015. DOI: 10.1002/adma.201501643.

[17] Z. Liu, Z.-S. Wu, S. Yang, R. Dong, X. Feng, and K. Müllen, "Ultraflexible in-plane microsupercapacitors by direct printing of solution-processable electrochemically exfoliated graphene", Advanced Materials, vol. 28, no. 11, pp. 2217-2222, 2016. DOI: 10.1002/adma.201505304.

[18] A. D. Smith, Q Li, A Anderson, A Vyas, V Kuzmenko, M Haque, L. G. H. Staaf, P Lundgren, and P Enoksson, "Toward cmos compatible wafer-scale fabrication of carbon-based microsupercapacitors for iot", Journal of Physics: Conference Series, vol. 1052, p. 012 143, 2018. DOI: $10.1088 / 1742-6596 / 1052 / 1 / 012143$.

[19] B. Sun, Y.-Z. Long, Z.-J. Chen, S.-L. Liu, H.-D. Zhang, J.-C. Zhang, and W.-P. Han, "Recent advances in flexible and stretchable electronic devices via electrospinning", J. Mater. Chem. $C$, vol. 2, no. 7, pp. 1209-1219, 2014. DOI: 10.1039/c3tc31680g.

[20] M. Mirjalili and S. Zohoori, "Review for application of electrospinning and electrospun nanofibers technology in textile industry", Journal of Nanostructure in Chemistry, vol. 6, no. 3, pp. 207213, 2016. DOI: $10.1007 / \mathrm{s} 40097-016-0189-\mathrm{y}$.

[21] X. Lu, C. Wang, F. Favier, and N. Pinna, "Electrospun nanomaterials for supercapacitor electrodes: Designed architectures and electrochemical performance", Advanced Energy Materials, vol. 7, no. 2, p. 1601 301, 2016. DOI: 10.1002/aenm.201601301.

[22] V. Aravindan, Y. L. Cheah, W. F. Mak, G. Wee, B. V. R. Chowdari, and S. Madhavi, "Fabrication of high energy-density hybrid supercapacitors using electrospun v2o5 nanofibers with a self-supported carbon nanotube network", ChemPlusChem, vol. 77, no. 7, pp. 570-575, 2012. DOI: $10.1002 / \mathrm{cplu} .201200023$.

[23] D. Tian, X. Lu, G. Nie, M. Gao, N. Song, and C. Wang, "Growth of polyaniline thorns on hybrid electrospun cnfs with nickel nanoparticles and graphene nanosheets as binder-free electrodes for high-performance supercapacitors", Applied Surface Science, vol. 458, pp. 389396, 2018. DOI: $10.1016 / \mathrm{j}$. apsusc.2018.07.103.

[24] N. Liu, G. Fang, J. Wan, H. Zhou, H. Long, and X. Zhao, "Electrospun pedot:pss-pva nanofiber based ultrahigh-strain sensors with controllable electrical conductivity", Journal of Materials Chemistry, vol. 21, no. 47, p. 18962, 2011. DOI: 10.1039/c1jm14491j.

[25] B. Bessaire, M. Mathieu, V. Salles, T. Yeghoyan, C. Celle, J.-P. Simonato, and A. Brioude, "Synthesis of continuous conductive pedot:pss nanofibers by electrospinning: A conformal coating for optoelectronics", ACS Applied Materials \& Interfaces, vol. 9, no. 1, pp. 950-957, 2016. DOI: 10.1021/acsami.6b13453.

[26] I. Teraoka, Polymer solutions. John Wiley \& Sons, 2004.

[27] D. Bloor and T. Blythe, Electrical properties of polymers. Cambridge University Press, 2008. 
[28] A. Elschner, Pedot: Principles and applications of an intrinsically conductive polymer. CRC, 2011.

[29] R. Balint, N. J. Cassidy, and S. H. Cartmell, "Conductive polymers: Towards a smart biomaterial for tissue engineering", Acta Biomaterialia, vol. 10, no. 6, pp. 2341-2353, 2014. DOI: 10.1016/j.actbio.2014.02.015.

[30] O. Dimitriev, D. Grinko, Y. Noskov, N. Ogurtsov, and A. Pud, "Pedot:pss films - effect of organic solvent additives and annealing on the film conductivity", Synthetic Metals, vol. 159, no. 21-22, pp. 2237-2239, 2009. DOI: 10.1016/j.synthmet.2009.08.022.

[31] S. van Reenen, M. Scheepers, K. van de Ruit, D. Bollen, and M. Kemerink, "Explaining the effects of processing on the electrical properties of pedot:pss", Organic Electronics, vol. 15, no. 12, pp. 3710-3714, 2014. DOI: 10.1016/j.orgel.2014.10.029.

[32] E. Barrera Raminrez, "Characterization of a pedot:pss electrolytic device using an in situ spectroelectrochemical technique", $\mathrm{PhD}$ thesis, Carleton University, 2013.

[33] D. Li and Y. Xia, "Electrospinning of nanofibers: Reinventing the wheel?", Advanced Materials, vol. 16, no. 14, pp. 1151-1170, 2004. DOI: 10.1002/adma.200400719.

[34] G. Taylor, "Disintegration of water drops in an electric field", Proceedings of the Royal Society A: Mathematical, Physical and Engineering Sciences, vol. 280, no. 1382, pp. 383-397, 1964. DOI: 10.1098/rspa.1964.0151.

[35] Y. Shin, M. Hohman, M. Brenner, and G. Rutledge, "Experimental characterization of electrospinning: The electrically forced jet and instabilities", Polymer, vol. 42, no. 25, pp. $09955-$ 09 967, 2001. DOI: 10.1016/s0032-3861(01)00540-7.

[36] P. Sharma and T. Bhatti, "A review on electrochemical double-layer capacitors", Energy Conversion and Management, vol. 51, no. 12, pp. 2901-2912, 2010. DOI: 10.1016/j.enconman. 2010.06.031.

[37] J. Kang, J. Wen, S. H. Jayaram, A. Yu, and X. Wang, "Development of an equivalent circuit model for electrochemical double layer capacitors (edlcs) with distinct electrolytes", Electrochimica Acta, vol. 115, pp. 587-598, 2014. DOI: 10.1016/j.electacta.2013.11.002.

[38] V. F. Lvovich, Impedance spectroscopy: Applications to electrochemical and dielectric phenomena, 1st ed. Wiley, 2013.

[39] J. R. Macdonald, Impedance spectroscopy: Theory, experiment, and applications, 2nd ed. Wiley, 2005.

[40] A. Allagui, T. J. Freeborn, A. S. Elwakil, and B. J. Maundy, "Corrigendum: Reevaluation of performance of electric double-layer capacitors from constant-current charge/discharge and cyclic voltammetry", Scientific Reports, vol. 7, p. 46828 , 2017. DOI: 10.1038/srep46828

[41] N. Elgrishi, K. J. Rountree, B. D. McCarthy, E. S. Rountree, T. T. Eisenhart, and J. L. Dempsey, "A practical beginner's guide to cyclic voltammetry", Journal of Chemical Education, vol. 95, no. 2, pp. 197-206, 2017. DOI: 10.1021/acs.jchemed.7b00361.

[42] M. A. Abud Kappel, F. C. Peixoto, G. M. Platt, R. P. Domingos, and I. N. Bastos, "A study of equivalent electrical circuit fitting to electrochemical impedance using a stochastic method", Applied Soft Computing, vol. 50, pp. 183-193, 2017. DOI: 10.1016/j.asoc.2016.11.030.

[43] M. Žic, "An alternative approach to solve complex nonlinear least-squares problems", Journal of Electroanalytical Chemistry, vol. 760, pp. 85-96, 2016. DOI: 10.1016/j.jelechem.2015. 11.015 . 
[44] A. S. Bondarenko, "Analysis of large experimental datasets in electrochemical impedance spectroscopy", Analytica Chimica Acta, vol. 743, pp. 41-50, 2012. DOI: 10.1016/j .aca.2012. 06.055 .

[45] M. R. Hasyim, D. Ma, R. Rajagopalan, and C. Randall, "Prediction of charge-discharge and impedance characteristics of electric double-layer capacitors using porous electrode theory", Journal of The Electrochemical Society, vol. 164, no. 13, A2899-A2913, 2017. DOI: 10.1149/ 2.0051713jes.

[46] M. Shoar Abouzari, F. Berkemeier, G. Schmitz, and D. Wilmer, "On the physical interpretation of constant phase elements", Solid State Ionics, vol. 180, no. 14-16, pp. 922-927, 2009. DOI: $10.1016 / \mathrm{j} . \mathrm{ssi} .2009 .04 .002$.

[47] R. German, P. Venet, A. Sari, O. Briat, and J. Vinassa, "Electrochemical double layer capacitors (supercapacitors) ageing impacts and comparison on different impedance models", EPE Journal, vol. 24, no. 3, pp. 6-13, 2014. DOI: 10.1080/09398368.2014.11742747.

[48] R. Jaeger, M. M. Bergshoef, C. M. I. Batlle, H. Schönherr, and G. Julius Vancso, "Electrospinning of ultra-thin polymer fibers", Macromolecular Symposia, vol. 127, no. 1, pp. 141-150, 1998. DOI: 10.1002/masy. 19981270119

[49] 2018. [Online]. Available: http://www.ampolymer.com/Mark-Houwink.html.

[50] C. Fernandes, L. MacEachern, and S. McGarry, "Electrospinning supercapacitors for microelectronic energy storage", in Materials Research Society 2018. MRS, 2018. [Online]. Available: https://mrsspring2018.zerista.com/event/member/466308.

[51] P. A. Small, "Some factors affecting the solubility of polymers", Journal of Applied Chemistry, vol. 3, no. 2, pp. 71-80, 2007. DOI: $10.1002 /$ jctb.5010030205

[52] L. D. Burke and P. F. Nugent, "The electrochemistry of gold: I the redox behaviour of the metal in aqueous media", Gold Bulletin, vol. 30, no. 2, pp. 43-53, 1997. DOI: 10.1007/bf03214756.

[53] K. Xu, "Nonaqueous liquid electrolytes for lithium-based rechargeable batteries", ChemInform, vol. 35, no. 50, 2004. DOI: 10.1002/chin.200450271

[54] T. Li, "Theoretical studies of lithium perchlorate in ethylene carbonate, propylene carbonate, and their mixtures", Journal of The Electrochemical Society, vol. 146, no. 10, p. 3613, 1999. DOI: $10.1149 / 1.1392523$.

[55] E. Smela, "Conjugated polymer actuators for biomedical applications", Advanced Materials, vol. 15, no. 6, pp. 481-494, 2003. DOI: 10.1002/adma.200390113. 